Published in final edited form as:

Adv Drug Deliv Rev. 2017 December 01; 122: 31-64. doi:10.1016/j.addr.2017.04.001.

\title{
Nanoparticles for drug delivery to the anterior segment of the eye
}

\author{
Dileep R. Janagam, Linfeng Wu, and Tao L. Lowe ${ }^{*}$ \\ Department of Pharmaceutical Sciences, University of Tennessee Health Science Center, \\ Memphis, TN 38163
}

\begin{abstract}
Commercially available ocular drug delivery systems are effective but less efficacious to manage diseases/disorders of the anterior segment of the eye. Recent advances in nanotechnology and molecular biology offer a great opportunity for efficacious ocular drug delivery for the treatments of anterior segment diseases/disorders. Nanoparticles have been designed for preparing eye drops or injectable solutions to surmount ocular obstacles faced after administration. Better drug pharmacokinetics, pharmacodynamics, non-specific toxicity, immunogenicity, and biorecognition can be achieved to improve drug efficacy when drugs are loaded in the nanoparticles. Despite the fact that a number of review articles have been published at various points in the past regarding nanoparticles for drug delivery, there is not a review yet focusing on the development of nanoparticles for ocular drug delivery to the anterior segment of the eye. This review fills in the gap and summarizes the development of nanoparticles as drug carriers for improving the penetration and bioavailability of drugs to the anterior segment of the eye.
\end{abstract}

\section{Graphical Abstract}

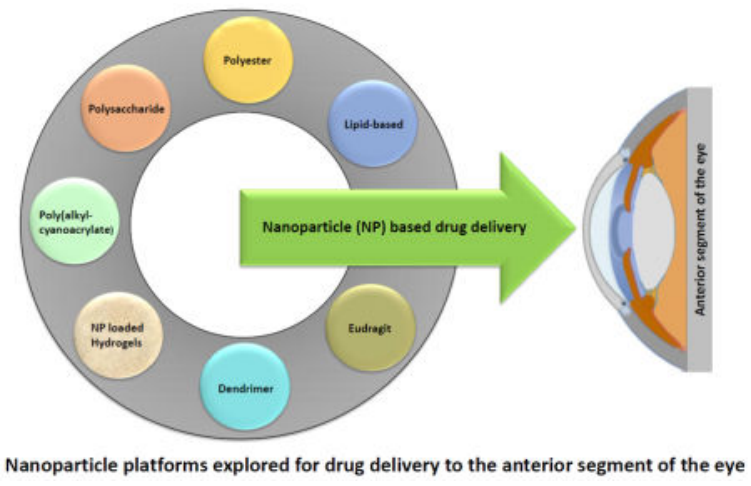

Nanoparticle platforms explored for drug delivery to the anterior segment of the eye

"Corresponding author at: Department of Pharmaceutical Sciences, University of Tennessee Health Science Center, Memphis, TN, 38163, USA. Tel.: 901-448-1087. tlowe4@uthsc.edu.

Publisher's Disclaimer: This is a PDF file of an unedited manuscript that has been accepted for publication. As a service to our customers we are providing this early version of the manuscript. The manuscript will undergo copyediting, typesetting, and review of the resulting proof before it is published in its final form. Please note that during the production process errors may be discovered which could affect the content, and all legal disclaimers that apply to the journal pertain. 


\section{Keywords}

nanoparticles; anterior segment of the eye; ocular barriers; poly(alkyl cyanoacrylate); polysaccharide; polyester; EUDRAGIT ${ }^{\circledR}$; lipid; dendrimer; contact lenses

\section{Introduction}

The eye is a delicate and very complicated organ having two main anatomical segments: the anterior segment and the posterior segment (Figure 1). The anterior segment is a part of the eyeball that is anterior to the lens and mainly consists of the cornea, conjunctiva, iris, lens, ciliary body, and the anterior portion of the sclera. It is further divided into two chambers, the anterior (between the posterior surface of the cornea and iris) and posterior (between the posterior surface of the cornea and the iris) chambers, which are connected by the opening of the pupil and filled with aqueous humor secreted by ciliary processes. The aqueous humor provides nutrients for lens and cornea, maintains intraocular pressure, and is replaced several times a day [1]. The diseases/disorders occurring in the anterior segment include cataracts, dry eye, congenital and developmental abnormalities, inflammatory diseases, infectious diseases, hereditary and degenerative diseases, glaucoma, tumors, injury, trauma, and ocular manifestations of systemic diseases. Clinically, these anterior segment diseases/disorders are more often treated by using eye drops or ointments. However, the efficacy of the eye drop and ointment treatments is low due to the existence of ocular barriers including the cornea and conjunctival barriers covering the ocular surface, and tear drainage (Figure 1) [1,2]. Nevertheless, local therapy via topical and periocular administrations is more favorable over therapies via systemic administration such as oral and intravenous administrations to manage ocular diseases. The first reason is that the eye has much fewer blood vessels and less blood flow than the whole body blood circulatory system so that the amount and rate of drugs to be cleared through local eye administration is much less than through systemic administration. The second reason is due to the presence of blood-aqueous barrier, which limits the drug penetration from the systemic circulation into the anterior segment of the eye. Recent advances in nanoparticles offer a great opportunity for efficient local delivery of drugs to the anterior segment. The advantages of nanoparticles include enhancing drug permeability across the blood-aqueous barrier and cornea, prolonging drug contact time with ocular tissues, delivering drugs to a specific tissue site in a controlled manner, protecting drugs from degradation and metabolism to enhance drug stability, sustaining drug release for weeks to months, having low to no toxicity and side effects, maintaining long shelf-life, and needing no reconstitution and no surgical removal. In this review, we will discuss the routes of administration and ocular barriers and summarize the up-to-date development of polymeric nanoparticles for drug delivery to the anterior segment of the eye.

\section{Routes of administration and ocular barriers for delivering drugs to the anterior segment}

There are four routes of administration for drugs to reach the anterior segment of the eye: topical, intracameral, subconjunctival, and systemic routes. Depending on the routes of administration, one or more ocular barriers need to be circumvented for drugs to reach the 
disease/action sites in the anterior segment of the eye. Table 1 lists the four routes of administration and their associated advantages and limitations. Figure 1 illustrates the structure of the anterior segment of the eye and ocular barriers for drug delivery. In the following, we will discuss the details of the four routes of administration and related ocular barriers.

Topical administration is the simplest, most convenient, self-administrable, non-invasive, and most dominant drug administration route for the management of anterior segment diseases/disorders. It is a local drug delivery method, avoiding the blood-aqueous barrier, and the side effects and first-pass metabolism that may occur in some systemically administrated drugs. Drugs administrated through the topical route are usually formulated into eye drops. Depending on the formulation and the drugs' physiochemical characteristics, drugs can reach various external (cornea, conjunctiva sclera) and internal (iris, ciliary body, aqueous humor, vitreous humor, retina) sites in the eye after topical instillation (Figure 2) [3, 4]. However, only $1-7 \%$ of the administered drugs can reach the aqueous humor due to the tear film, and cornea and conjunctiva barriers [5-7]. The tear film is the first obstacle faced for topically administered drugs. It consists of three layers: an outermost lipid layer, a thicker aqueous middle layer and an innermost mucin layer (Figure 1). The tear film is created by tears which are composed of water, electrolytes, and many different proteins that work together to promote healing and fight infection. A human tear has a total volume of about $7 \sim 30 \mu \mathrm{L}$ with a turnover rate of $0.5 \sim 2.2 \mu \mathrm{L} / \mathrm{min}$, and tear film has a rapid restoration time of 2 3 $\min$ [8]. Due to the fast turnover rate and time of tear film, the topically administered eye drops are quickly washed away and drained into nasolacrimal duct after instillation. The cornea is the second ocular barrier limiting the penetration of exogenous substances into the eye. It is composed of five layers: epithelium, Bowman's membrane, stroma, Descemet's membrane, and endothelium (Figure 1). The layers which form substantial barriers to drug penetration are epithelium, stroma, and endothelium. The superficial corneal epithelium is composed of multiple layers of stratified squamous nonkeratinized epithelial cells. It limits the permeation of hydrophilic drugs through the cornea due to the hydrophobicity of the epithelium and the presence of tight junctional proteins between the corneal epithelial cells [3,9]. The inner layer next to the corneal epithelium is the stroma. The stroma is comprised of glycosaminoglycans and collagen fibrils in lamellar structures, and it has a hydrophilic environment. It restricts the penetration of lipophilic drugs through the cornea [3]. The innermost layer of the cornea is monolayer corneal endothelium composed of hexagonal-shaped endothelial cells. This corneal endothelium is leakier than the epithelium and allows the penetration of macromolecules into the aqueous humor on the other side [3]. Overall, the cornea acts as a physical barrier to hydrophilic drugs due to the superficial corneal epithelial layers, and to lipophilic drugs due to the stroma. Besides the cornea route, topically administered drugs can be absorbed into the anterior segment through a non-cornea route: the conjunctiva/sclera pathway, as the conjunctiva has a larger surface area than the cornea $\left(17 \mathrm{vs} . \sim 1 \mathrm{~cm}^{2}\right)[5,6,10,11]$. Conjunctiva is a rate-limiting barrier for permeation of water-soluble drugs $[12,13]$ due to rapid drug elimination by conjunctival blood and lymphatic flow. After escaping from conjunctival elimination, drugs penetrate through the sclera to reach the anterior segment (trans-scleral pathway). The sclera has large surface area and relatively high permeability 
than the cornea, and the trans-scleral permeation mainly depends on the size of the drug molecules rather than their lipophilicity $[3,14,15]$.

Intracameral administration is a method for direct injection of drugs into the anterior chamber in the anterior segment of the eye [16]. It is a local drug delivery method, avoiding the side effects and the first-pass metabolism that may occur in some systemically administrated drugs. It also avoids the cornea, conjunctiva, and blood-aqueous barriers. Therefore, intracameral injection can deliver drugs to the anterior segment relatively easily with high efficiency and was expected to achieve 300 to 600 times more aqueous humor drug level than topical application [17, 18]. After intracameral injection, the mechanism for drugs to reach tissues in the anterior segment is via diffusion and the bulk flow of aqueous humor. In general, the literature supports the safety of intracamerally injected antibiotics such as vancomycin, moxifloxacin, and cephalosporins [18-20]. In addition, the intracameral injection technique was shown to be useful in the off-label use of anti-vascular endothelial growth (anti-VEGF) agents and triamcinolone acetonide for the management of neovascularization in the treatment of neovascular glaucoma and endothelial allograft rejection after penetrating keratoplasty, respectively [21, 22]. However, intracameral antibiotics usually need reconstitution including dilution and other special preparations which require sterilization, preservative-free, and proper concentration and dose. If incorrect dosing and preparation occur, corneal endothelial toxicity and toxic anterior segment syndrome become big concerns $[18,20]$.

Subconjunctival administration places drugs into the subconjunctival space around the outside of the sclera, and then drugs penetrate through the sclera and reach the anterior segment. It is a minimally invasive and effective route for delivering drugs to the anterior segment, avoiding the cornea and blood-aqueous barriers, and the side effects and first-pass metabolism that may occur in some systemically administrated drugs. It is a local drug delivery method, and can offer sustained drug delivery depending on formulations or devices. However, the subconjunctival route has the limitation of possible loss of drugs to the systemic circulation due to the drainage via the conjunctival blood and lymphatic vessels $[14,23]$.

Systemic administration can deliver drugs to the anterior segment, but with low bioavailability for conventional ophthalmic formulations such as solutions and suspensions, due to the presence of the blood-aqueous barrier. The two layers that comprise the bloodaqueous barrier (BAB) are the endothelium of the iris/ciliary blood vessels and the nonpigmented epithelium of the ciliary body (Figure 1) [1,3]. Because of the presence of the tight junctional complexes in both these layers, BAB restricts the penetration of drug molecules from the blood into the aqueous humor [3, 24]. In order to achieve therapeutic levels of drugs in the aqueous humor, high doses are necessary, and high doses can cause adverse systemic side effects so that the systemic administration route is rarely used to treat anterior segment disease/disorders. For clarification, we would like to specifically mention that the term bioavailability in this review means the rate and extent of drug available at the target anterior ocular site such as tear fluid, corneal or conjunctival tissue, aqueous humor, iris, etc. after drug administration [25-28]. Three pharmacokinetics (PK) parameters that are commonly used for assessing ocular bioavailability are: $C_{\max }$, the maximum drug 
concentration achieved at target ocular site; $T_{\max }$, the time required to achieve the $C_{\max }$; and $A U C$, the area under curve (drug concentration in ocular tissue vs time curve) [27, 29-31]. $A U C$ is the most commonly used parameter to describe ocular bioavailability [30], and noted as $A U C_{(0-\mathrm{t})}$ and $A U C_{(0-\infty)}$ for time 0 to $t$ and 0 to infinity, respectively [31].

\section{Nanoparticles for drug delivery to the anterior segment}

As discussed above, the ocular bioavailability of topically administrated drugs is limited due to several factors such as small anatomical space, baseline and reflex lachrymation tear drainage, metabolic degradation of the drugs, and other anatomical and physiological barriers in the eye [7, 24, 32-35]. Appropriate concentrations of the drug are needed at targeted sites in the eye to achieve therapeutic effects of the drugs. To obtain the appropriate concentrations, frequent instillation of eye drops containing the drugs is the common method. However, the frequent dosing method can cause toxic side effects and damage to ocular tissues [3]. It is important and necessary to develop technologies that can deliver drugs to the targeted sites in the eye with low or no frequent dosing. Therapeutic targets for drugs to treat anterior eye segment - related diseases can be located in extra- or intraocular tissues [36]. Based on the therapeutic target areas, the goals of drug delivery to the anterior segment of the eye can be sub-divided into two categories, a) to improve the bioavailability the drugs at extraocular tissues to alleviate the signs and symptoms caused by ocular surface inflammatory disorders of cornea and conjunctiva, such as dry eye syndrome and allergic diseases; and b) to enhance the bioavailability of the drugs in the intraocular tissues to treat infections and complex, vision-threatening diseases such as glaucoma or intraocular inflammation (uveitis). Nanoparticles have large surface areas and can be made of many types of materials with multifunctional surface groups. These materials hold great promise in helping drugs to reach targeted sites to achieve the stated two purposes of drug delivery to the anterior segment of the eye [2,37-44]. The reasons are that nanoparticles can be entrapped in the ocular mucus layer and then bioadhesive polymer chains/materials that form the nanoparticles can intimately interact with the extraocular tissues to prolong the residence time of drugs loaded in the nanoparticles and reduce drug drainage and thus improve the bioavailability the drugs at extraocular tissues [33]. Furthermore, nanoparticles can also penetrate through ocular surface tissues to deliver encapsulated drugs to intraocular tissues so that improved bioavailability of the drugs at the intraocular tissues can be achieved [45]. The penetration depends on the size, charge, architecture, surface chemistry, and hydrophilicity/hydrophobicity of the nanoparticulate systems [46-48]. Table 2 summarizes some of the important characteristics/properties of the nanoparticles and corresponding characterization techniques used to determine those properties.

The penetration mechanisms for nanoparticles into the interior segment of the eye have not been deeply studied; however, the commonly accepted endocytosis mechanism in explaining nanoparticles' tissue penetration was also observed by Robinson and his colleagues when they used fluorescence microscopy to study the penetration of poly(butyl cyanoacrylate) nanoparticles across the ex vivo rabbit cornea and conjunctiva [46, 49-54]. The most commonly used in vitro and ex vivo models for evaluating the corneal penetration have been summarized by Agarwal et al [55]. Additionally, nanoparticles can protect drugs from 
degradation and sustain release of drugs which can also increase the bioavailability of the drugs in both extra- and intraocular tissues.

In the following discussions, we will systematically review the progress and challenges in the development of polymeric nanoparticles and their composites with hydrogel matrix for delivering drugs to the anterior segment. These polymeric nanoparticles are made of poly(alkyl cyanoacrylate), polysaccharides, polyesters, EUDRAGIT ${ }^{\circledR}$ polymers and lipids, whose chemical structures are illustrated in Tables 2-5. There are other strategies such as imprinted contact lenses [56], vitamin E based contact lenses [57], and nanowafer-based systems [58-61] that have been used for extended ocular drug delivery to the anterior segment; however, these strategies are not discussed in this review as the main focus of this review is nanoparticles rather than other systems for drug delivery to the anterior segment.

\subsection{Poly(alkyl cyanoacrylate) nanoparticles}

Poly(alkyl cyanoacrylate) (PACA, Table 2) nanoparticles emerged in the early 1980s and have been extensively studied for drug delivery to the brain and eye [62]. PACA nanoparticles were prepared through emulsion and interfacial polymerizations from monomers, or nanoprecipitation and emulsion-solvent evaporation from presynthesized PACA polymers [62]. Due to the excellent adhesive properties of alkyl cyanoacrylates, PACA nanoparticles were the first nanoparticles investigated for topical ocular drug delivery to the eye [54, 63-68]. Poly (2-hexyl cyanoacrylate) nanoparticles were found to have 4-fold greater accumulation in inflamed albino rabbit eye tissues than healthy tissue. [63, 66]. Hydrophobic drugs such as pilocarpine [64] and acyclovir [69], and hydrophilic drugs such as betaxolol hydrochloride [67] and amikacin sulfate [68], were successfully loaded into PACA nanoparticles. Poly(butyl cyanoacrylate) PBCA nanoparticles improved myotic response to pilocarpine in albino rabbits [64]; increased the concentration of amikacin sulfate in the cornea and aqueous humor in the presence of dextran 70000 [68]; and enhanced the absorption of positively charged betaxolol hydrochloride with decreasing its surface zeta potential and the subsequent antiglaucoma activity of betaxolol hydrochloride, depending on the amount of the drug released [67]. Table 6 summarizes various drug-loaded PACA nanoparticles and their characteristics. Since PACA nanoparticles were found to cause cell lysis and cornea damage in 90's [54, 62], PACA nanoparticles have been seldom investigated for ocular drug delivery although improved ocular toleration of PACA nanoparticles was observed in the six-hour tolerability study by coating PACA nanoparticles using poly(ethylene glycol) (PEG) polymers [69]. Researchers switched to other more biocompatible polymers for the development of polymeric nanoparticles for ocular delivery as discussed below.

\subsection{Polysaccharide nanoparticles}

Polysaccharides are long chain carbohydrate molecules having a large number of reactive functional groups varying in chemical composition. Their molecular weights vary widely due to the diversity of their structures and properties. Common hydrophilic functional groups present in the polysaccharides are hydroxyl, carboxyl and amino groups. These functional groups can form hydrogen bonds with mucosa to become mucoadhesive [70]. Due to the hydrogen bonds, when mucoadhesive polysaccharides are added in the eye drops, 
the drug's retention time in the tear film and anterior chamber is prolonged, and the clearance of the drug is controlled by the mucus turnover rate rather than the tear turnover rate and is much slower [33,71-78]. Besides the mucoadhesive property, nature-originated polysaccharides such as chitosan and its derivatives, alginate, hyaluronic acid, gum cordia, and carboxymethyl tamarind kernel polysaccharide (Table 3), possess other appealing properties such as acceptable biocompatibility, excellent ocular tolerance $[79,80]$, biodegradability [81], and ability to enhance drug membrane permeability both in vitro [82] and in vivo [83]. The combination of these properties makes polysaccharides versatile biopolymers for ocular drug delivery [71, 84-89]. Drugs such as cyclosporine A (CsA) [90], dorzolamide hydrochloride [91, 92], pramipexole hydrochloride [91], acyclovir [93], 5flurouracil [94], carteolol [92], gatifloxacin [95], betamethasone sodium phosphate [96], gene [97], pilocarpine [98], econazole nitrate [99], natamycin [100], daptomycin [89], amphotericin B [101], celecoxib [102], timolol maleate [103, 104], fluconazole [105], sodium diclofenac [106], and tropicamide [107] were loaded into nanoparticles made of polysaccharides for ocular delivery. The release of the drugs from these hydrophilic polymer nanoparticle matrices relies on various factors such as polymer hydration, solvent penetration, drug diffusion, drug dissolution, and/or polymer erosion [95]. The discussions below detail the studies carried out on polysaccharide-based nanoparticles for drug delivery to the anterior segment of the eye.

3.2.1. Chitosan nanoparticles_Chitosan has arisen as a promising material for the improvement of drug delivery to the ocular mucosa and is most extensively studied polysaccharide. Chitosan is a cationic linear polysaccharide copolymer of 1,4-(2-amino-2deoxy- $D$-glucopyranose) and 1,4-(2-acetamido-2-deoxy- $D$-glucopyranose). The mucoadhesive nature of chitosan is related to the interaction between the positively charged amino groups of chitosan and the negatively charged residues of sialic acid in the mucus of cornea and conjunctiva [108] along with other interactions such as hydrogen bonds [109]. Another characteristic of chitosan is its penetration enhancement properties, as it can transiently open the tight junctions between cells $[85,110]$. Chitosan nanoparticles are often synthesized via ionic gelation techniques using sodium tripolyphosphate as a crosslinker $[80,85,86,111,112]$. The benefits associated with chitosan-based nanoparticles include their ability to contact intimately with the corneal and conjunctival surfaces, and thus enhance drug delivery to external ocular tissues while minimizing the toxicity of the drugs to the internal ocular tissues and blood stream (through systemic absorption). Therefore, the chitosan-based nanoparticles show promising potential for the treatment of external ocular diseases [90]. Table 7 summarizes various drug-loaded chitosan nanoparticles and their characteristics.

Alonso and associates first reported the study of exploring chitosan nanoparticles for delivery of drugs to the ocular surface in 2001 [90] and subsequently conducted extensive studies on chitosan nanoparticles [80, 90, 97, 112]. Synthesized chitosan nanoparticles were reported to have an average size of several hundred nanometers depending on the synthetic conditions, and an almost constant zeta potential of about +35 to $+37 \mathrm{mV}[80,90,93,102$, 112] which was higher than $+30 \mathrm{mV}$ implying that the nanoparticles were stable due to the prevention of the aggregations of the nanoparticles by the surface charges [93]. In vitro

Adv Drug Deliv Rev. Author manuscript; available in PMC 2018 December 01. 
studies of chitosan nanoparticles in simulated fluids containing mucus components (mucin and lysozyme) showed that the presence of lysozyme did not compromise the stability of chitosan nanoparticles in the tear fluid. The interaction of chitosan nanoparticles with mucin did not significantly affect the viscosity of the mucin dispersion [112]. These chitosan nanoparticles were not inherently toxic to Chang conjunctival cell line at concentrations up to $2 \mathrm{mg} \cdot \mathrm{ml}^{-1}$ (Figure 3) [112] and spontaneously immortalized epithelial cell line from normal human conjunctiva (IOBA-NHC) at concentrations up to $1 \mathrm{mg} \cdot \mathrm{ml}^{-1}$ for up to $24 \mathrm{~h}$ [80]. In vivo examination of topically administrated chitosan nanoparticles in rabbits showed that the nanoparticles did not cause inflammation, tissue alteration, and damage to the epithelial layer of the rabbit cornea as shown in Figure $4[80,94]$. Another in vivo investigation revealed that fluorescein-containing chitosan nanoparticles significantly accumulated in the rabbit cornea and conjunctiva after topically instilled to the cul-de-sac of conscious rabbits, evidenced by fluorescence intensity measurements and confocal image (Figures 5 and 6) [112] [80]. Further study of chitosan nanoparticles as a drug carrier for cyclosporine A (CsA) revealed that the nanoparticles showed a high in vitro burst release of the drug CsA (62\% within 15 min) in sink conditions due to the rapid dissolutions of both the hydrophilic chitosan nanoparticles and the drug present at or close to the surface of the nanoparticles. But the authors speculated that the burst release would be minimized in vivo due to the absence of such a significant dilution process upon administration [90]. In vivo investigation of the ocular disposition of CsA showed that the therapeutic concentrations of CsA were attained in the external ocular tissues (cornea and conjunctiva) within $48 \mathrm{~h}$ after topical instillation of CsA-loaded chitosan nanoparticles in rabbits, while the CsA levels in the inner ocular structures (iris/ciliary body and aqueous humor), blood and plasma were negligible or undetectable. The therapeutic concentration levels in the external ocular tissues were found to be significantly higher after the instillation of CsA-loaded chitosan nanoparticles compared with the control CsA-containing chitosan (not chitosan nanoparticle) solution and aqueous CsA suspension [90]. These results demonstrated that chitosan nanoparticles could be used as carriers to enhance the therapeutic index of clinically challenging drugs such as CsA with the potential application at the extraocular level. Besides CsA, chitosan nanoparticles have also been studied for ocular delivery of other drugs such as dorzolamide hydrochloride [91, 92], pramipexole hydrochloride [91], acyclovir [93], 5-flurouracil [94], carteolol [113], and ketorolac tromethamine [114]. The in vitro release profiles of the drugs dorzolamide hydrochloride, pramipexole hydrochloride, acyclovir, 5-flurouracil, and carteolol showed a high burst release [91, 93, 94] as that of CsA [90]. The mucoadhesive strength of dorzolamide hydrochloride-, and pramipexole hydrochloride-loaded chitosan nanoparticles decreased with increasing the drug content due to the decreased amount of chitosan that was available for adhesion and the increased size of the nanoparticles [91]. Topically instilled 5-fluorouracil-loaded chitosan nanoparticles exhibited significantly higher maximum concentration $C_{\max }(\sim 2.7$ times $)$ and bioavailability (AUC $\sim 3.5$ times during $8 \mathrm{~h}$ ) of fluorouracil in the aqueous humor of rabbits when compared with the drug solution alone [94]. Whole body images by gamma scintigraphy revealed that chitosan nanoparticles prolonged the retention of cateolol on the corneal and conjunctival surfaces of rabbits to significantly enhance its ocular hypotensive effect in $24 \mathrm{~h}$ study [92].

Adv Drug Deliv Rev. Author manuscript; available in PMC 2018 December 01. 
3.2.2. Chitosan-based hybrid nanoparticles-Due to the hydrophilic nature of chitosan, nanoparticles made of chitosan alone have limited encapsulation potential for hydrophobic drugs [115]. To further improve the drug entrapment, as well as specific targeting, controlled release and toxicity reduction capability of nanoparticles made of chitosan alone, a second polymer or oligomer such as sodium alginate, hyaluronic acid, cholesteryl 3-hemisuccinate, dextran sulfate, sulfobutyl ether-cyclodextrin, Carbopol ${ }^{\circledR}$, or lecithin (Tables 3 and 4) has been added into the chitosan nanoparticles for ocular drug delivery applications $[95,99,101,103,115-120]$. These second polymers/oligomers have plenty of anionic carboxylic, sulfonate or phosphate groups which can form ionic interaction with cationic chitosan so that they can act as crosslinkers like tripolyphosphate which is commonly used for crosslinking chitosan. The crosslinking can increase the tightness of chitosan nanoparticles, and subsequently increase the ocular retention time of the loaded drugs and/or the ocular penetration of the chitosan nanoparticles, decrease the initial burst, and increase the ocular bioavailability of the drugs [95, 99]. Table 8 summarizes various drug-loaded chitosan-based hybrid nanoparticles and their characteristics.

Polyionic complex nanoparticles made of hybrid chitosan and sodium alginate could continuously release antibiotic gatifloxacin [95] and betamethasone sodium phosphate [96] for 24-72 $\mathrm{h}$ in vitro and generated smaller initial burst for gatifloxacin than the chitosan or alginate alone [95]. An ex vivo permeability study showed that chitosan-alginate nanoparticles could penetrate through the rabbit sclera. In vivo studies of these nanoparticles in rabbits showed sustained release of betamethasone sodium phosphate for 12-24 h depending on the formulations when compared with the drug solution alone which disappeared after $2 \mathrm{~h} \mathrm{[96].} \mathrm{Zhu} \mathrm{et} \mathrm{al.} \mathrm{[121]} \mathrm{synthesized} \mathrm{thiolated} \mathrm{chitosan} \mathrm{and} \mathrm{evaluated} \mathrm{the}$ potential of thiolated chitosan-sodium alginate nanoparticles for ocular delivery. Results from their in vitro cytotoxicity study showed that the thiolated chitosan-sodium alginate nanoparticles were not cytotoxic to human corneal epithelial cells (HCE). Compared with chitosan-sodium alginate nanoparticles, thiolated chitosan-sodium alginate nanoparticles were smaller, more stable and adhesive, showed greater intracellular uptake by HCE cells, and delivered more FITC (model drug) into corneas [121].

Alonso and associates investigated the effectiveness of hyaluronic acid (HA) modified chitosan (chitosan-HA) nanoparticles for delivering genes to the cornea and conjunctiva [97, 122]. The zeta potential values of the chitosan-HA nanoparticles varied from positive to negative with increasing HA content. The transfection results demonstrated that the chitosan-HA nanoparticles were able to render high transfection levels to immortalized human corneal epithelial and normal human conjunctival cells (up to $15 \%$ of cells transfected), without affecting cell viability. After topical instillation into rabbit eyes, the chitosan-HA nanoparticles were internalized into the conjunctiva more than the cornea. Confocal images revealed that the internalization of chitosan-HA nanoparticles was due to fluid endocytosis, and the process was mediated by hyaluronan receptor CD44 (Figure 7). The nanoparticles were evenly distributed in corneal epithelial cells, but unevenly in conjunctival cells with more intense localization in the apical and basolateral regions. Vyas and associates [103] evaluated the effectiveness of these chitosan-HA nanoparticles for delivering timolol maleate and dorzolamide hydrochloride to the eye for the management of 
glaucoma. As expected, the addition of HA in the nanoparticles showed increased ex vivo mucoadhesion and transcorneal permeation of the drug through excised goat cornea when compared with chitosan nanoparticles alone. Both chitosan-HA and chitosan nanoparticles showed in vitro burst release. Most of the loaded timolol maleate was released out of the chitosan/chitosan-HA nanoparticles as timolol was molecularly dispersed in the nanoparticles, whereas only $20 \%$ of the loaded dorzolamide hydrochloride was released due to its crystalline form. The timolol/dorzolamide hydrochloride loaded chitosan-HA nanoparticles did not cause any apparent ocular irritation to albino rabbits. They reduced intraocular pressure significantly more in the fellow eyes but less in the contralateral eyes of albino rabbits after $24 \mathrm{~h}$ of instillation [103]. The chitosan-HA nanoparticles caused higher intraocular pressure (IOP) lowering and less systemic absorption of the two drugs timolol and dorzolamide hydrochloride than the chitosan nanoparticles and the drug solutions after the instillation [103].

Yuan X et al. [118] synthesized amphiphilic conjugates of hydrophilic chitosan and hydrophobic cholesterol $(\mathrm{CH})$ and obtained $\mathrm{CH}$ modified chitosan (chitosan- $\mathrm{CH}$ ) nanoparticles through self-aggregation, and evaluated their potential for drug delivery to the ocular surface. The formed nanoparticles had an average particle size of about $200 \mathrm{~nm}$ and an average zeta potential of about $44 \mathrm{mV}$. They showed good dispersion over the entire precorneal area of rabbits following topical administration. While some of the chitosan-CH nanoparticle suspension was observed to be drained into lacrimal sac through the lacrimal duct, $71.4 \%$ of the chitosan- $\mathrm{CH}$ nanoparticles were well retained at the precorneal area at 112 min post-administration due to the bioadhesion ability of chitosan. Subsequently, the chitosan-CH nanoparticles retained at the cornea and conjunctiva, but would hardly permeate into the iris/ciliary body and reach to the posterior segment of the eye due to the tight barrier of the cornea. Hydrophobic drug CsA was entrapped in these nanoparticles using a simple dialysis method. In comparison with the chitosan nanoparticles alone, chitosan-CH nanoparticles enhanced both the CsA loading content and loading efficiency of chitosan nanoparticles (9\% and $73 \%$, vs. $6.2 \%$ and $41.8 \%$, respectively). The chitosan-CH nanoparticles showed sustained release of CsA for over $48 \mathrm{~h}$ compared with the chitosan nanoparticles ( $24 \mathrm{~h}$ ), and less initial burst than the chitosan nanoparticles alone (60\% released in $4 \mathrm{~h}$ vs. $62 \%$ released in $15 \mathrm{~min}$ ) as shown in Figure 8 [118, 123]. The reason for the less initial burst was probably due to the hydrophobic nature of the cholesterol component. This group also showed that further incorporation of hydrophobic poly(lactic acid) into the chitosan- $\mathrm{CH}$ nanoparticles could significantly improve the loading efficiency of rapamycin by $\sim 4$ fold and slow rapamycin release by reducing the percentage of rapamycin released at $12 \mathrm{~h}$ from $85 \%$ to $50 \%$ [124]. Furthermore, the chitosan-CH/PLA nanoparticles improved the immunosuppressive effect of rapamycin in corneal transplantation in New Zealand rabbits as effectively as and even slightly better than rapamycin suspension in terms of the medial survival time of the corneal allografts (27.2 \pm 1.03 vs. $23.7 \pm 3.20$ days).

Hybrid nanoparticles made of chitosan and Carbopol ${ }^{\circledR}$ with $294 \mathrm{~nm}$ particle size were used to load pilocarpine [98]. The subsequent in vitro and in vivo studies demonstrated that these hybrid nanoparticles could slowly release pilocarpine for $24 \mathrm{~h}$ to decrease the pupil diameter of rabbits (miosis effect) more effectively than pilocarpine in liposome, gel and eye drop 
formulations. Hybrid nanoparticles made of chitosan and dextran sulfate with around 400 $\mathrm{nm}$ particle size and around $40 \mathrm{mV}$ zeta potential were stable in the presence of lysozyme for at least $4 \mathrm{~h}$ and showed at least 60 min prolonged adherence to the ex vivo porcine corneal surface [117]. After topical instillation of mucoadhesive chitosan-dextran sulfate nanoparticles ( $400 \mathrm{~nm}$ size/ $+48 \mathrm{mV}$ zeta potential; FITC labelled), the nanoparticles showed prolonged retention on porcine ocular surface for more than $4 \mathrm{~h}$, and could be endocytosed into the ex vivo porcine corneal epithelial cells via clathrin-dependent pathway (Figure 9) [125]. Furthermore, after $6 \mathrm{~h}$ of topical administration, theses chitosan-dextran sulfate nanoparticles were observed to not be able to penetrate through corneal epithelium into stroma (Figure 10) [125]. Beta-cyclodextrin and its derivatives have a unique property of forming inclusion complexes with hydrophobic drugs to increase these drugs' water solubility due to their hydrophobic central cavity made of seven glucose units [126, 127]. In ocular application, sulfobutyl ether $\beta$-cyclodextrin was used to make hybrid nanoparticles with chitosan to form inclusion complexes with econazole nitrate to increase the water solubility of econazole nitrate. The resulting econazole nitrate-containing complexes showed improved bioavailability and prolonged 8 hours' steady antifungal effect while econazole nitrate solution could provide an antifungal effect for only 3 hours which also dramatically decreased with time [99].

Hybrid nanoparticles made of chitosan and lecithin had a particle size of $\sim 100 \mathrm{~nm}$ and zeta potential value of around 27 to $43 \mathrm{mV}$, and were used for controlled release of natamycin [100], amphotericin B [101], and celecoxib [102]. In vitro release of natamycin showed a biphasic release profile with an initial burst release $\sim 41.23 \%$ in $2 \mathrm{~h}$ followed by a slow release $\sim 64.22 \%$ in $7 \mathrm{~h}$ [100]. However, in vitro release of celecoxib showed $24 \mathrm{~h}$ sustained release without initial burst [102]. In vivo ocular pharmacokinetics study of natamycin in New Zealand rabbits showed that the hybrid chitosan-lecithin nanoparticles increased the $A U C_{(0-\infty)}$ of natamycin 1.47-fold and decreased the clearance of natamycin 7.4-fold compared with the marketed natamycin suspension [100, 101]. In vivo ocular pharmacokinetics study of amphotericin B in albino rabbits showed that the hybrid chitosanlecithin nanoparticles significantly improved the bioavailability ( 2.04-fold) and precorneal residence time ( $\sim 3.36$-fold) of amphotericin B compared with amphotericin B solution (Fungizone ${ }^{\circledR}$ ) available in the market [101].

3.2.3. Nanoparticles made of other polysaccharides-Other polysaccharides such as pectin, gum cordia, carboxymethyl tamarind kernel polysaccharide, galactomannan polysaccharide, and hyaluronic acid have also been used to make nanoparticles for ocular drug delivery [104-107, 128]. In an ex vivo study, pectin nanoparticles were found to significantly increase the permeability of timolol maleate across the excised goat cornea $200 \%$ more than the conventional timolol maleate ophthalmic solution [104]. On the other hand, gum cordia and carboxymethyl tamarind kernel polysaccharide nanoparticles were found to not enhance the permeation of loaded drugs fluconazole and tropicamide across the goat cornea as compared with the respective drug ophthalmic solutions [105, 107]. The authors speculated that even though these two types of nanoparticles did not increase the drug ex vivo corneal permeability, they might be able to provide sustained drug delivery in vivo due to their prolonged residence time in the cul-de-sac. Horvat et al. demonstrated that 
the gel formulations made of nanosized crosslinked sodium hyaluronate, prepared by crosslinking via the carboxyl groups of the HA chains with a diamine using a carbodiimide technique, exhibited the highest capability of mucoadhesion compared with the gels made of linear HA derivatives (linear sodium hyaluronate, zinc hyaluronate). The explanations were that the interchain crosslinking structure and nanosize dimensions might enable the crosslinked sodium hyaluronate to interpenetrate easily and form secondary bonds with the mucin. Despite their differences in chemical and physical structures, all the above three types of nanoparticles showed an initial burst release of model drug sodium diclofenac at

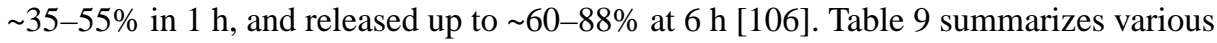
drug-loaded nanoparticles made of other polysaccharides and their characteristics.

\subsection{Polyester nanoparticles}

Polyesters such as poly(lactide) (PLA), poly(lactide-co-glycoside) (PLGA) and poly( $\varepsilon$ caprolactone) (PCL) (Table 2) are synthetic polymers which are biodegradable with good biocompatibility. These polymers have been extensively used to fabricate implants, microparticles, and nanoparticles for controlled drug release and targeted drug delivery. In particular, nanoparticles fabricated from PLA, PLGA and PCL have been evaluated for drug delivery to the eye [129-135]. Drug or non-drug-loaded PLA, PLGA and PCL nanoparticles have been fabricated using solvent displacement (i.e. nanoprecipitation) [132-134, 136138], and oil-in-water (o/w) emulsion solvent evaporation techniques [135, 139]. The resulting nanoparticles have particle sizes in the range of a hundred to a couple of hundred nanometers and bear negative zeta potentials. Hydrophobic drugs such as sparfloxacin, indomethacin, cyclosporine A, natamycin and flurbiprofen and hydrophilic drugs such as brimonidine and diclofenac sodium were encapsulated in these polyester nanoparticles. Below are detailed discussions about how PLA, PLGA and PCL and their derivative nanoparticles have been investigated to mask drug irritation to the eye, improve drug loading in the formulations, increase drug retention time in the precorneal area, and/or enhance drug penetration into/across the cornea or the conjunctiva-sclera barrier to reach the inner anterior segment [130, 132, 134, 137, 139-142].

Self-assembled polymeric micelles were synthesized from amphiphilic copolymers bearing a hydrophobic poly(2-methyl-2-carboxytrimethylene carbonate-co-D,L-lactide) backbone and a hydrophilic poly(ethylene glycol)-azide pendant chain [143]. GRGDS peptide was conjugated to the self-assembled micelles to target corneal epithelial cells through RGD receptors. In vitro evaluation of the binding affinity of these GRGDS-modified micelles to rabbit corneal epithelial cells showed that GRGDS-modified micelles could significantly inhibit the attachment of corneal epithelial cells to GRGDS-coated plates, indicating these micelles could be used for targeted drug delivery to the injured eye. PLGA nanoparticles prepared by nanoprecipitation were tested for ex vivo permeability across the cornea of New Zealand rabbits by Vega et al. [134]. The results showed that the PLGA nanoparticles considerably increased the penetration of flurbiprofen across the cornea by 4-fold over free flurbiprofen PBS (pH 7.4) solution and by about 2-fold over the commercial Ocuflur ${ }^{\mathrm{TM}}$ eye drops [134]. Corneal hydration study indicated that the corneas were not damaged during the 6 h ex vivo corneal permeability study. Similar drug penetration enhancement was observed on ex vivo goat corneas for sparfloxacin-loaded PLGA nanoparticles [137]. Further, in vivo 
ocular retention study on New Zealand rabbits using scintigraphy studies revealed that radiolabeled sparfloxacin-loaded PLGA nanoparticles were cleared at a much slower rate and retained for a much longer duration at the corneal surface than commercial sparfloxacin eye drops. This was evidenced by the facts that after topical instillation radioactivity was detected in the systemic circulation (kidney and bladder) at $6 \mathrm{~h}$ with radiolabeled sparfloxacin eye drops but not with radiolabeled sparfloxacin-loaded PLGA nanoparticles; and radioactive counts at corneal surface within the first $0.5 \mathrm{~h}$ quickly decreased with radiolabeled sparfloxacin eye drops but remained nearly constant with radiolabeled sparfloxacin-loaded PLGA nanoparticles [137]. Since PLGA nanoparticles are hydrophobic and negatively charged at the surface, they are non-mucoadhesive in nature [137]. Therefore, the observed long retention for the nanoparticles would probably due to the particle size of the nanoparticles (180-190 nm) [137, 144]. In addition to the PLGA nanoparticle size effect, the poly(vinyl alcohol) stabilizer was also found to help to increase the nanoparticle retention time by increasing the viscosity of the nanoparticle dispersion due to the hydrophilic nature of the poly(vinyl alcohol) polymer [137, 140]. Diclofenac sodium-loaded PLGA and poly (lactide-co-glycolide-leucine) polymeric nanoparticle suspensions with size $\sim 125-190 \mathrm{~nm}$, and zeta potential $\sim-25 \mathrm{mV}$ showed a biphasic release pattern of the drug: one initial fast release ( $50 \%$ in first $2 \mathrm{~h}$ ) followed by a second slow-release phase for up to $14 \mathrm{~h}$, and the particles were observed to be free of any irritant effect on cornea, iris, and conjunctiva for $24 \mathrm{~h}$ after topical application in rabbit eyes [142]. PCL nanocapsules have been studied for topical ocular drug delivery by Alonso and co-workers [136, 140, 145]. These PCL nanocapsules were made from PCL, lecithin and Miglitol 840 using solvent displacement technique. The fluorescent dye loaded PCL nanocapsules with a mean size of $252 \mathrm{~nm}$ have enhanced the penetration of rhodamine $6 \mathrm{G}$ through the corneal epithelial cells of New Zealand rabbits by endocytosis ex vivo, and were selectively internalized into the corneal epithelium cells (vs. the conjunctival cells) after topical instillation to the cul-de-sac of fully awake New Zealand rabbits [140]. PCL nanocapsules loaded with indomethacin had an average particle size of $238 \mathrm{~nm}$ and a zeta potential of $-39 \mathrm{mV}$ and were well tolerated by New Zealand rabbits, and significantly increased the indomethacin concentrations in the cornea ( 3.2-fold increase in AUC) and aqueous humor ( 4.3-fold increase in AUC) than commercial eye drop Indocollyre after topical instillation to the cul-de-sac of the right eyes in New Zealand rabbits [136]. After PCL nanoparticles had been functionalized with poly$D$-glucosamine, the obtained nanoparticles prolonged the release of natamycin for up to $8 \mathrm{~h}$ in vitro, and significantly increased relative bioavailability ( 6.03-6.39-fold) compared to the commercial natamycin suspension $\left(\right.$ Natamet $\left.^{\circledR}\right)$ [138]. Ibrahim et al. loaded brimonidine into PLA (molecular weight $152 \mathrm{kDa}$ ), PLGA 75:25 (molecular weight 66-107 kDa) and PCL (molecular weight $14 \mathrm{kDa}$ ) nanoparticles which had 117 131 nm mean particle size, $-18 \sim-28.11 \mathrm{mV}$ zeta potential (Figure 11) [135]. The nanoparticles could encapsulate 68$78 \%$ of brimonidine, and the encapsulation efficiency increased in the order of PLGA $<$ PLA $<$ PCL with increasing the hydrophobicity of the polymers and the consequent solid-state solubility of the hydrophobic brimonidine freebase in the polymers. The in vitro release rates of brimonidine from the nanoparticles increased in the order of PCL $>$ PLGA>PLA due to the decrease of the molecular weights of the polymers used in the nanoparticle preparations. Ibrahim et al. further embedded the brimonidine-loaded nanoparticles into methyl cellulose-based gels and demonstrated that the resulting 
brimonidine-nanoparticle-gel systems had significant better IOP lowering effect than brimonidine-containing Alphagan $\mathrm{P}^{\circledR}$ eye drops (effective duration 15.2-23.2 vs. 7-7.4 h) after instillation in BXD (boxed molecular dynamics) mice [135].

To increase the precorneal residence time and consequent ocular bioavailability of drugloaded PLA, PLGA, and PCL polyester nanoparticles, mucoadhesive polymers chitosan [89, 131, 136, 146], cholesterol modified chitosan [124], Carbopol ${ }^{\circledR}[139]$ and hyaluronic acid (HA) $[129,147]$ were used to form a coating layer on the nanoparticles. Among these mucoadhesive polymers, chitosan is the most extensively exploited one. Chitosan coating was introduced into the polyester nanoparticles during the nanoparticle synthesis by using a chitosan-containing aqueous solution. This chitosan coating significantly shifted the zeta potential of the nanoparticles from negative (uncoated) to positive (coated) value [124, 131, $136,146]$. The positively charged nanoparticles can interact with the negatively charged mucus layer of the tear film and the negatively charged outer layer of the cornea to prolong the retention time of drugs on the corneal surface and increase the penetration of drugs across the cornea. EX vivo permeability studies showed that by loading rhodamine $6 \mathrm{G}$ into chitosan-coated PCL nanoparticles or simply mixing rhodamine $6 \mathrm{G}$ with blank chitosancoated PCL nanoparticles, the transport of rhodamine $6 \mathrm{G}$ across the rabbit cornea was significantly enhanced more (1.9-3.3-fold in $4 \mathrm{~h}$ ) compared with uncoated PCL nanoparticles [131]. Similar observation was reported on the ex vivo study of the permeability of rhodamine 6G-loaded chitosan-coated PLGA nanoparticles across the goat cornea [146]. In vitro studies demonstrated that the chitosan coating on PCL nanoparticles did not modify indomethacin release kinetics from the PCL nanoparticles, but strongly helped to increase the indomethacin concentrations in the cornea and aqueous humor of rabbits more than uncoated PCL nanoparticles and commercial eye drop Indocollyre after topical instillation [136]. However, when PCL nanoparticles were coated with neutral PEG, the transport of loaded rhodamine $6 \mathrm{G}$ across the ex vivo rabbit cornea was not increased [131]; when PCL nanoparticles were coated with positively charged PLL, the ocular bioavailability of indomethacin in rabbit cornea and aqueous humor was not increased either [136], in comparison with uncoated PCL nanoparticles. The possible explanation for the enhanced corneal transport and ocular bioavailability by the chitosan coating but not the PLL and PEG coatings might be that the chitosan significantly increased the zeta potential of the PCL nanoparticles from -39.9 to $37.1 \pm 1.8 \mathrm{mV}$ [136] more than the PLL and PEG $(27.9 \pm 3.5$ and $-40.2 \pm 1.7 \mathrm{mV}[131,136]$, respectively), and had strong mucoadhesive nature. A recent study showed that cholesterol-modified chitosan/PLA nanoparticles mainly remained on the cornea and conjunctiva too for about $150 \mathrm{~min}$ after topical instillation, which is about 2-6 times higher than the drug suspension [124]. Researchers also used negatively charged Carbopol ${ }^{\circledR}$ (crosslinked polyacrylic acid) to coat PLGA nanoparticles, and obtained slightly larger (393 vs. $219 \mathrm{~nm}$ ) and more negatively charged nanoparticles compared with uncoated PLGA nanoparticles [139]. In vitro uptake studies showed that the Carbopol $^{\circledR}$ coated PLGA nanoparticles were taken up by L929 mouse fibroblast cells more than the corresponding uncoated PLGA nanoparticles (40\% vs. $33.2 \%$ after 6 h exposure). This increased cellular uptake might be attributed to the adhesive properties gained by the nanoparticle through the Carbopol ${ }^{\circledR}$ coating. The bioadhesive properties imparted by Carbopol $^{\circledR}$ were probably due to the entanglement between Carbopol ${ }^{\circledR}$ and mucin layer 
covering the corneal epithelium and the hydrogen bonding between the carboxylic groups of Carbopol ${ }^{\circledR}$ and the glycoproteins of the mucin layer [139]. Barbault-Foucher et al. coated PCL nanospheres with hyaluronic acid (HA) and investigated the effect of cationic surfactants stearylamine and benzalkonium chloride (BKC) on the coating [129]. Their results showed that both of the cationic surfactants enhanced the adhesion of HA on PCL nanosphere surface, and BKC had a higher effect than stearylamine (41.6 vs. $26.4 \mathrm{mg}$ $\mathrm{HA} / \mathrm{mg}$ PCL). Yenice et al. further studied the effects of the PCL/BKC nanospheres with and without HA coating on CsA corneal delivery in healthy rabbits [147]. Both PCL/BKC and HA coated PCL/BKC nanospheres increased CsA corneal delivery 10-15 times more than CsA solution in castor oil. Furthermore, the HA coated PCL/BKC nanospheres delivered much more CsA in the cornea than the uncoated PCL/BKC nanospheres (11.423.0 vs. $5.9-15.5 \mathrm{ng} / \mathrm{mg}$ tissue) $4 \mathrm{~h}$ post-topical administration. Phenylboronic acid that can form a complex with cis-diol groups of sugar units, such as sialic acid, ample in mucin at physiological $\mathrm{pH}[148,149]$, [added comma] was used to functionalize the surface of $\operatorname{poly}(D, L$-lactide)-b-dextran nanoparticles to increase the precorneal residence time of the nanoparticles [150]. The surface functionalization with phenylboronic acid led to change in the size and zeta potential of the nanoparticles from 45.8 to $28.6 \mathrm{~nm}$ and -2.63 to $-31.3 \mathrm{mV}$, respectively; and high mucin binding of the nanoparticles: $1.18 \pm 0.02 \mathrm{mg}$ per $\mathrm{mg}$ nanoparticles, which was much greater that the values attained with other types of mucoadhesive nanoparticles such as chitosan-based nanoparticles $(\sim 0.25 \mathrm{mg}$ per $\mathrm{mg}$ nanoparticles) and thiolated nanoparticles ( $\sim .13 \mathrm{mg}$ per $\mathrm{mg}$ nanoparticles). Slit-lamp examination and histopathology observations in rabbits demonstrated that phenylboronic acid surface functionalized poly $(D, L$-lactide)- $b$-dextran nanoparticles $(\sim 35.6 \mathrm{~nm})$ loaded with and without CsA (12\% loading) did not show any detectable irritation or inflammatory reactions after weekly administration for up to 12 weeks. The nanoparticles sustained release CsA for 5 days in vitro, and the released CsA showed efficacy in treating experimental dry eye-induced mice in vivo: once a week administration of CsA loaded nanoparticles achieved both the elimination of inflammatory infiltrates and recovery of the ocular surface, whereas thrice a day administration of commercial product Restasis ${ }^{\circledR}$ (Cyclosporine Ophthalmic Emulsion, $0.05 \%$ ) only showed clearance of the inflammatory processes [150]. Table 10 summarizes various drug-loaded polyester nanoparticles and their characteristics.

\subsection{EUDRAGIT ${ }^{\circledR}$ polymer nanoparticles}

The hypothesis that the positive charges bearded by nanoparticles can facilitate an effective adhesion to the ocular surface stimulated the development of nanoparticles made of cationic EUDRAGIT ${ }^{\circledR}$ for topical ocular drug delivery [151-160]. EUDRAGIT ${ }^{\circledR}$ is made of poly(ethyl acrylate-methyl methacrylate-chlorotrimethyl-aminoethyl methacrylate) (Table 2) containing quaternary groups between $4.5-6.8 \%$ and $8.8-12 \%$ for RS and RL grades, respectively. Nanoparticles made of EUDRAGIT ${ }^{\circledR}$ were fabricated by nanoprecipitation [155], spontaneous emulsification [156], and quasi-emulsion solvent diffusion techniques $[151,152]$. These EUDRAGIT ${ }^{\circledR}$ nanoparticles demonstrated good ocular tolerance in rabbit eyes, and did not cause any inflammation in the ocular tissues [151, 155, 161, 162]. Pignatello and his group encapsulated ibuprofen and flurbiprofen into EUDRAGIT ${ }^{\circledR}$ RS nanoparticles and tested their effect in preventing miosis caused by surgical traumas [151, 152] and ocular inflammation [151]. Compared with the corresponding eye drops, 
EUDRAGIT $^{\circledR}$ RS nanoparticles enhanced the presence of the loaded drugs (ibuprofen and flurbiprofen) in the aqueous humor of rabbit eyes [151,152]. This increase of drug concentration and prolonged drug persistence in the aqueous humor provided a high capacity to inhibit miosis and inflammation in the anterior segment of the rabbit eye. These results were attributed to the enhanced drug retention at the eye surface due to the encapsulation of the drugs in the nanoparticles, and a progressive and continuous release of the incorporated drugs from the nanoparticles adhered on the cornea surface [151,152]. Amikacin loaded EUDRAGIT ${ }^{\circledR}$ RS 100/RL 100 nanoparticles were prepared and assessed for in vitro, in vivo and pharmacokinetic performances [163]. They had a mean particle size 122-242 nm, and zeta potential $29-46 \mathrm{mV}$ depending on the proportion between RS 100 and RL 10. In vivo ocular retention study by scanning electron microscope revealed that the EUDRAGIT ${ }^{\circledR}$ nanoparticles exhibited corneal adherence for prolonged $6 \mathrm{~h}$ after instillation in healthy male albino rabbits. The EUDRAGIT ${ }^{\circledR}$ nanoparticles significantly increased the $C_{\max } \sim 1.733$ times and $A U C_{0-12 \mathrm{~h}} \sim 2.12$ times of amikacin in aqueous humor more than the commercial ophthalmic solution of amikacin sulfate [163]. Hybrid EUDRAGIT ${ }^{\circledR}$ nanoparticles were prepared using o/w emulsification-solvent evaporation method from mixtures of PLGA and EUDRAGIT ${ }^{\circledR}$ RL with different weight ratios (75:25, 50:50 and 25:75) [139]. The particle size of the obtained hybrid nanoparticles slightly increased with the increase of the PLGA content, and the nanoparticles' zeta potential was positive and was not affected by the EUDRAGIT $^{\circledR}$ RL content. An in vitro uptake study showed that the hybrid EUDRAGIT ${ }^{\circledR}$ nanoparticles (75:25) was taken up by $\mathrm{L} 929$ cells much more than Carbopol ${ }^{\circledR}$ coated PLGA and uncoated PLGA nanoparticles (50\% vs. $40 \%$ and 33.2\%, respectively after 6 h) (Figure 13). An in vivo pharmacokinetic study revealed that the hybrid EUDRAGIT ${ }^{\circledR}$ nanoparticles (75:25) gave the highest $A U C_{0-24 \mathrm{~h}}\left(972.59 \mathrm{ng} \cdot \mathrm{h} \cdot \mathrm{g}^{-1}\right)$ and $C_{\max }\left(366.30 \mathrm{ng} \cdot \mathrm{g}^{-1}\right)$ of cyclosporine $\mathrm{A}$ in the rabbit tear, compared with the Carbopol ${ }^{\circledR}$ coated PLGA $\left(A U C_{0-24} \mathrm{~h}\right.$ $776.57 \mathrm{ng} \cdot \mathrm{h} \cdot \mathrm{g}^{-1}$ and $C_{\max } 211.08 \mathrm{ng} \cdot \mathrm{g}^{-1}$ ) and uncoated PLGA $\left(A U C_{0-24 \mathrm{~h}} 490.42 \mathrm{ng} \cdot \mathrm{h} \cdot \mathrm{g}^{-1}\right.$ and $\left.C_{\max } 126.12 \mathrm{ng} \cdot \mathrm{g}^{-1}\right)$ nanoparticles. The higher bioavailability in case of Hybrid EUDRAGIT $^{\circledR}$ nanoparticles could be the result of ionic interaction between the negatively charged mucin layer at the eye surface and the cationic EUDRAGIT ${ }^{\circledR}$ RL nanoparticles where these interactions offer the possibilities of prolonging the residence time of the formulation in the eye. Hyaluronic acid (HA) was used to coat EUDRAGIT ${ }^{\circledR}$ nanoparticles aiming for prolonged ocular delivery of gatifloxacin/prednisolone biotherapy [156]. These HA coated EUDRAGIT ${ }^{\circledR}$ nanoparticles were prepared by either coating preformed EUDRAGIT ${ }^{\circledR}$ nanoparticles with HA or applying the HA coating during the synthesis of EUDRAGIT $^{\circledR}$ nanoparticles via a spontaneous emulsification method. The latter process produced much larger HA coated nanoparticle $(678.00 \mathrm{~nm})$ compared with the process of coating preformed nanoparticles $(460.30 \mathrm{~nm})$. However, the both types of HA coated nanoparticles had similar zeta potential value of about -45 to $-47 \mathrm{mV}$. The HA-coated nanoparticles provided significantly increased and prolonged drug concentrations in the cornea ( $C_{\max } 5.2$-fold, $A U C_{0-6 \mathrm{~h}} 7.9$-fold) and aqueous humor ( $C_{\max } 1.8$-fold, $\mathrm{AUC}_{0-6 \mathrm{~h}}$ 1.8 -fold) of rabbit eyes in vivo compared with the commercial eye drops. However, no comparison between the coated nanoparticles and uncoated nanoparticles was reported in the paper [156]. Currently, in the literature, there is no report about the limitation of EUDRAGIT $^{\circledR}$ or EUDRAGIT ${ }^{\circledR}$ nanoparticles. Based on general knowledge, one obvious limitation of EUDRAGIT ${ }^{\circledR}$ is that the polymer poly(ethyl acrylate-methyl methacrylate- 
chlorotrimethyl-aminoethyl methacrylate) for making EUDRAGIT ${ }^{\circledR}$ nanoparticles is not biodegradable. Further studies about the efficacy and side effects of EUDRAGIT ${ }^{\circledR}$ nanoparticles are needed in the future. Table 11 summarizes various drug-loaded EUDRAGIT $^{\circledR}$ based nanoparticles and their characteristics.

\subsection{Lipid nanoparticles}

Lipid nanoparticles including solid lipid nanoparticles and nanostructured lipid carriers emerged in the 90s as an alternative drug delivery system to liposomes and polymeric nanoparticles [164, 165]. Lipid nanoparticles have an advantage over liposomes in terms of low cost for large scale production and less storage and drug leakage issues [166, 167]. They have advantages over biodegradable polymeric nanoparticles in terms of less significant toxicity and acidity problems [166, 167]. Lipid nanoparticles have been extensively investigated in the academic and pharmaceutical industry as carriers for dermal, mucosal, oral, intravenous/parenteral, pulmonary and ocular delivery of small molecule drugs, peptides/proteins and genes [168]. Lipid nanoparticles usually have a size in the range of $50 \sim 1000 \mathrm{~nm}$ and remain in a solid state at body temperature. They are synthesized from various lipids such as lipid acid, monoglycerides, diglycerides, triglycerides, glyceride mixtures and waxes using high-pressure homogenization, microemulsion, solvent emulsionevaporation/diffusion, double emulsion, high-speed stirring and/or ultrasonication techniques [168-171].

The application of solid lipid nanoparticles (SLN) for ocular drug delivery traced back to 2002 when Cavalli et al. reported their study on tobramycin-loaded solid lipid nanoparticles [172]. Cavalli et al. synthesized tobramycin-loaded SLN from stearic acid, Epikuron 200, sodium taurocholate and water using a warm o/w microemulsion method. They obtained 70 80 nm SLN that were well tolerated by rabbit eyes and did not cause any ocular irritation. An in vivo ocular pharmacokinetics study showed that the SLN could deliver tobramycin in the aqueous humor of rabbit eyes, and increased tobramycin $C_{\max } 1.5$-fold, $T_{\max } 8$-fold and $A U C_{0-6} \mathrm{~h}$-fold more than an equal dose of commercial tobramycin eye drops (Tobral ${ }^{\circledR}$ ) after topical instillation. This enhanced tobramycin bioavailability by SLN might be attributed to the entrapment and retaining of the SLN in the mucin layer covering the corneal epithelium, and the Epikuron 200 component in the SLN which helped to enhance the corneal penetration of tobramycin. Caramella and her group developed a CsAloaded SLN using Compritol 888 ATO (glyceryl behenate) as a solid lipid, and Poloxamer 188 and Tween 80 as surfactants via a high shear homogenization and ultrasound method $[173,174]$. They obtained optimized Compritol-SLN with a particle size of $225 \mathrm{~nm}$ with and without CsA and a zeta potential value of $-16.9 \mathrm{mV}$ and $-23.6 \mathrm{mV}$ with and without CsA, respectively. The CsA loading efficiency could reach as high as $95.6 \%$. They found that Compritol-SLN nanoparticles sterilized at $110{ }^{\circ} \mathrm{C}$ showed no cytotoxicity to corneal epithelial cells (RCE) in vitro and did not induce ocular irritation in rabbit determined by the Draize test. However, Compritol-SLN sterilized at $120^{\circ} \mathrm{C}$ leaked out surfactant Tween 80 and induced cytotoxicity to REC cells in vitro. Compared with CsA suspension, the Compritol-SLN nanoparticles significantly enhanced the ex vivo penetration of CsA through the porcine cornea 10-fold more. Further, an in vivo study demonstrated that CsA could penetrate into the aqueous humor at a therapeutic level two hours after topical instillation of 
CsA-loaded SLN into rabbit eyes. To improve SLN's interaction with and internalization in corneal cells, Caramella and her group added cationic chitosan during the SLN synthesis [175]. Başaran et al. also [176] reported that octadecylamine-modified cationic SLN with size $248 \pm 0.33 \mathrm{~nm}$ and zeta potential $-50.30 \pm 0.78 \mathrm{mV}$ could continuously release CsA in the aqueous humor and vitreous humor of sheep eye for more than $48 \mathrm{~h}$ after topical instillation. Leonardi et al. fabricated melatonin-encapsulated cationic SLN using Softisan 100 as the main lipid matrix, didecyldimethylammonium bromide as a cationic lipid, stearic acid or palmitic acid as a lipid modifier, tween 80 as a surfactant, and lipid didecyldimethylammonium bromide as a cationic surface lipid [177]. The obtained nanoparticles had a size range of 150 300 nm and polydispersity index (PDI) $<0.35$ with positive surface charge, and caused significant IOP lowering in albino rabbits for about $24 \mathrm{~h}$. Using compritol and palmitic acid as lipid carriers, Poloxamer 188 and soya lecithin as surfactants, and sodium taurocholate as a co-surfactant, Kumar et. al prepared SLN of size range $139-334 \mathrm{~nm}$ and zeta potential $>-30 \mathrm{mV}$ for the ocular delivery of voriconazole [178]. The SLN exhibited sustained release of voriconazole ( $60-80 \%$ in $12 \mathrm{~h}$ vs. $\sim 100 \%$ in $6 \mathrm{~h}$ for drug suspension). Histopathology (Figure 14) studies of excised goat cornea and HET-CAM studies (Figure 15) treated with the SLN, irritant (0.1 N) and control $(0.9 \%$ $\mathrm{NaCl})$ demonstrated that the SLN were non-irritant to the cornea [178]. In vivo pharmacokinetics study of the SLN in rabbits revealed that the SLN significantly increased the bioavailability of voriconazole with $C_{\max } 1.4$-fold, $A U C_{0-12 \mathrm{~h}}$ 2-fold, and mean residence time (MRT) 1.2-fold increases in comparison to the plain drug suspension [178]. Sharma et. al. prepared GMS based SLN and found that the ${ }^{99 \mathrm{~m}}$ Tc labeled SLN greatly prolonged celecoxib retention on the rabbit ocular surface than the drug aqueous suspension ( $20 \%$ vs. $75 \%$ drop in the intensity) by using gamma scintigraphy [179]. The GMS based SLN with size around $200 \mathrm{~nm}$ could significantly increase the permeation of celecoxib across the rabbit cornea compared to celecoxib aqueous suspension (permeation $8.21 \pm 0.67 \%$ vs. $4.61 \pm 0.71 \%)[179]$.

Nanostructured lipid carriers (NLC) appeared in the late 90s and early this century to overcome the limitations associated with solid lipid nanoparticles such as limited drug loading, the risk of gelation and drug leakage during storage [170]. In NLC, spatially incompatible liquid lipids were incorporated into the solid lipids to generate structure defects in the crystals for the accommodation of drugs [180, 181]. In 2008, Pan and his group first reported NLC for ocular drug delivery [182]. They fabricated NLC from Compritol ATO, Gelucire 44/14, stearylamine, Miglyol 812, Transcutol and/or Cremophor EL using a melted-ultrasonic method. They found that the obtained NLC with an average particle size of $69 \mathrm{~nm}$ and a zeta potential value of $+28.9 \mathrm{mV}$ were well tolerated by rabbit eyes, increased the ex vivo permeability of ibuprofen across the rabbit cornea 4.9-fold, and increased the $A U C, C_{\max }$, and $T_{\max }$ of ibuprofen in the rabbit aqueous humor 3.99-, 3.25and 3.2-fold, respectively, compared with conventional ibuprofen eye drops. The enhanced bioavailability in the aqueous humor might be attributed to the Gelucire 44/14 and Transcutol P components in the NLC which enhanced the permeability of ibuprofen across the cornea, and the stearylamine component in the NLC which prolonged the precorneal retention of ibuprofen. Following this pioneering report, more groups devoted effort to the development of NLC for ocular drug delivery including anterior eye segment drug delivery 
[180, 183-190]. One attempt was to further improve the precorneal retention and/or transcorneal penetration of drugs by incorporating mucoadhesive polymers/ligands in NLC. Chitosan oligosaccharides [186], partially deacetylated water-soluble chitosans [188], and N-acetyl-L-cysteine functionalized chitosan (CS-NAC) [191] were used to coat preformed NLC fabricated from Compritol 888 ATO, Gelucire 44/14, and Miglyol 812 using meltedemulsification and ultrasonication techniques. The chitosan coating layer increased the sizes of the NLC $\sim 21-40 \%$ and rendered positive zeta potential to the NLC. The chitosan-coated and uncoated NLC did not cause any irritation to rabbit corneas and conjunctivas. The chitosan coating increased the precorneal retention of drug flurbiprofen and the penetration of the drug across the ex vivo rabbit cornea, depending on the types and amounts of the chitosan coatings $[186,188]$. Mucoadhesive thiolated PEG stearate was also incorporated into a NLC fabricated from Precifac ATO $^{\circledR} 5$ and Miglyol ${ }^{\circledR} 840$ for ocular drug delivery $[185,189]$. The thiolated PEG stearate did not change the NLC's size (around $60 \mathrm{~nm}$ ) and zeta potential $(-12$ to $-18 \mathrm{mV}$ ). CsA loaded NLC with and without thiolated PEG stearate could sustain release of CsA for more than 2 days in vitro and the thiolation of NLC could slightly decrease the in vitro drug release rate [189]. The thiolated NLC were non-irritant to rabbit eyes, increased the residence time of CsA on the rabbit cornea surface to $6 \mathrm{~h}$, at least 1.5 times more than non-thiolated NLC, and delivered $~ 2-15$ times more CsA to the cornea, conjunctiva, iris-ciliary body, aqueous humor and tear fluid than castor oil solution eye drops of CsA, NLC and PEG-NLC $24 \mathrm{~h}$ after instillation into the lower cul-de-sac of rabbit eyes. The CsA loaded thiolated PEG searate NLCs could deliver $934.96 \mathrm{ng} / \mathrm{g}$ of CsA to the irisciliary body of rabbit eyes with good immunomodulation effect. These results suggested a direct correlation between the thiol group composition and the mucoadhesive behavior of the NLC: the thiol group formed disulfide bonds with the cysteine-rich subdomains of mucus glycoproteins to enhance the mucoadhesion of the NLC $[185,192]$. Liu et al. fabricated CSNAC coated NLC that had particle size $\sim 50$ to $88 \mathrm{~nm}$ and zeta potential $\sim-20$ to $+22 \mathrm{mV}$ [191]. They found that the ex vivo and in vivo transcorneal permeability of curcumin was increased in the order of solution eye drops < NLC < chitosan-coated NLC < CS-NACcoated NLC with $C_{\text {max }}, A U C_{0-\infty}$ and $M R T_{0-\infty}$ increasing 3.88-, 5.97- and 1.27-fold, 5.28-, 12.25- and 2.19-fold, and 8.88-, 29.88- and 2.9-fold, respectively, in comparison to the drug eye drops [191]. They explained that the reason for the NLC to increase the transcorneal permeability of curcumin more than the drug solution was attributed to the presence of the lipid matrix in the NLC which could adhere to corneal epithelial cells to increase the residence time of curcumin on the cornea surface. The reason for the chitosan-coated NLC to increase the transcorneal permeability of curcumin more than the NLC alone was attributed to the electrostatic interaction of the positively charged chitosan-coated NLC with the cornea surface which could lead to temporary loosening of tight junctions between corneal epithelial cells to increase the penetration of the drug. The highest permeability observed with the CS-NAC-coated NLC was because of the potential opening of tight junctions between corneal epithelial cells through inhibition of protein tyrosine phosphatase by thiolation and the mucoadhesive nature of the N-acetyl-L-cysteine coated on the NLC. Amphipathic octadecyl-quaternized carboxymethyl chitosan was also used as a cationic material to be coated on NLC to improve the transcorneal permeability and ocular bioavailability of curcumin [193]. The obtained nanoparticles had particle size $158 \mathrm{~nm}$ and zeta potential $+36.5 \mathrm{mV}$, increased the in vivo corneal permeability of curcumin in rabbits

Adv Drug Deliv Rev. Author manuscript; available in PMC 2018 December 01. 
more than the NLC alone with $C_{\max }, A U C_{0-\infty}$ and $M R T_{0-\infty}$ 1.3-,2.4- and 2.3-fold vs. 1.2-, 1.5- and 1.8-fold increase, respectively, in comparison to the drug eye drops. The above results indicated that $\mathrm{N}$-acetyl-L-cysteine functionalized chitosan was more promising than amphipathic octadecyl-quaternized carboxymethyl chitosan to be used as a coating for NLC for improving the ocular bioavailability of curcumin [191, 193].

Ban et al. modified dexamethasone-loaded lipid nanoparticles made of soy/lecithin by chitosan coating and observed that the modification resulted in a 1.5-fold increase in apparent permeability coefficient of the drug dexamethasone [194]. The chitosan-modified lipid nanoparticles increased the dexamethasone ocular bioavailability more than the unmodified ones in rabbits with $C_{\max }, T_{\max }$ and $A U C_{0-24 \mathrm{~h}}$ increasing 2.37-, 12 and 4.69fold vs. 1.17-, 10 and 2.12-fold, respectively in comparison to dexamethasone eye drops [194]. Liu et al. evaluated liquid crystalline nanoparticles (LCNP) for the delivery of tetrandrine using components including glyceryl monoolein, poloxamer 407, water, Gelucire 44/14 and amphipathic octadecyl-quaternized carboxymethyl chitosan [195]. In vitro corneal permeation test demonstrated that LCNP enhanced the apparent permeability coefficient of the drug by 2.03-fold compared to the drug solution. LCNP showed prolonged ocular residence time in vivo, and increased the ocular bioavailability of tetrandrine in rabbits by 2.85-fold compared to the drug solution [195]. Table 12 summarizes various drug-loaded lipid-based nanoparticles and their characteristics.

\subsection{Dendrimers}

Dendrimers are nanoscale, highly branched, and reactive three-dimensional macromolecules with a high degree of molecular uniformity, narrow molecular weight distribution, specific size, and intriguing structural properties such as internal voids and cavities and a highly functional terminal surface formed by amino, carboxyl and/or hydroxyl groups [196, 197]. Since Tomalia et al. first synthesized polyamidoamine (PAMAM) dendrimers in the mid 1980's [198], dendrimers have been extensively studied for drug and gene delivery and diagnosis because drugs, genes, diagnostic agents, and targeting moieties can be encapsulated in the central core or physicochemically conjugated to the terminal surface of the dendrimers [199-202]. However, dendrimers have been explored for ocular drug delivery in only recent ten years [41, 43] since the first report by Vandamme et al. in 2005 [203]. Hydrophilic drugs pilocarpine nitrate [203], carteolol [204], timolol maleate [205, 206] gatifloxacin [207] and hydrophobic drugs tropicamide [203], acetazolamide [208], and brimonidine $[205,206]$ have been loaded into dendrimers for ocular drug delivery. In Vandamme et al.'s study, tropicamide and pilocarpine nitrate-loaded PAMAM dendrimers were evaluated for drug residence time on the cornea, and miotic and mydriatic activities, after instillation in the eyes of New Zealand albino rabbits [203]. The results showed that the dendrimer aqueous solutions had comparable $\mathrm{pH}$, osmotic pressure, viscosity, surface tension, and refractive index as Carbopol $980 \mathrm{NF}(0.2 \%$ w/v) eye drops with no disturbance in the visual acuity. The mean corneal residence times $(\sim 4-5 \mathrm{~h})$ of the aqueous solutions of PAMAM dendrimers with generations $\mathrm{G} 1.5(\mathrm{COOH}), \mathrm{G} 2(\mathrm{OH})$ and $\mathrm{G} 4(\mathrm{OH})$ were comparable to that of the Carbopol $980 \mathrm{NF}(0.2 \% \mathrm{w} / \mathrm{v})$ eye drops, while that of the dendrimer $\mathrm{G} 2(\mathrm{NH} 2)$ was significantly shorter. The PAMAM dendrimers with carboxyl or hydroxyl terminal surface groups increased the miotic effect of pilocarpine nitrate with 
1.39-1.64-fold increase in $A U C$ and the mydriatic activity of tropicamide with 1.13-1.34fold increase in AUC in comparison with $1 \%$ drug in phosphate buffer [203]. Spataro et al. developed phosphorus-containing dendrimers that had one quaternary ammonium salt as core and carboxylic acid as terminal groups for delivering carteolol to treat glaucoma [204]. They loaded carteolol into the dendrimers through ion pair interaction between the amino group of the neutral carteolol and the carboxyl group of the dendrimers. The water solubility of the obtained catanionic (saline) species decreased with increasing the generation of the dendrimers (G0-soluble; G1, G2-poorly soluble). The dendrimers were not irritant to the eyes of albino rabbits for at least $8 \mathrm{~h}$ after installation. Upon instillation, G0 dendrimers did not improve concentration; however, G2 dendrimers caused a 2.5 times increase in the concentration of carteolol in the aqueous humor in comparison with the drug suspension alone [204]. Durairaj et al. reported the development of dendritic polyguanidilyated translocators (DPT) as an ocular drug delivery vehicle to enhance the solubility and permeability of gatifloxacin [207]. The DPT nanoparticles that they synthesized had a size of around $346 \mathrm{~nm}$ and increased the water solubility of gatifloxacin by 4 -fold. The nanoparticles enhanced the ex vivo permeability of gatifloxacin across the bovine sclerachoroid-RPE by $40 \%$, and increased the in vitro killing effect of gatifloxacin to methicillinresistant Staphylococcus aureus by a factor of 3. In comparison with the drug solution alone, the nanoparticles increased the bioavailability of gatifloxacin in the cornea and conjunctiva of New Zealand white rabbits by $\sim 2$ - and $~ 13$-fold, respectively; and prolonged the retention time of gatifloxacin in the rabbit aqueous humor from 2 to $24 \mathrm{~h}$ [207]. Mishra $\mathrm{V}$ et al. investigated the effectiveness of acetazolamide-loaded G5.0 poly(propylene imine) dendrimers in lowering the intraocular pressure in normotensive adult male New Zealand albino rabbits [208]. The dendrimers increased the corneal residence time of acetazolamide by 5-7-fold depending on the dendrimer concentration, and prolonged the IOP-lowering effect of acetazolamide from $2 \mathrm{~h}$ to $\sim 4 \mathrm{~h}$ in comparison with acetazolamide solution alone. Table 13 summarizes various drug-loaded dendrimer nanoparticles and their characteristics.

\subsection{Nanoparticle-based composite systems}

The above discussed nanoparticle systems have proven promising in terms of increasing the drug residence time, improving the ocular penetration of drugs and enhancing the ocular bioavailability of the drugs. However, as nanoparticles have small size and drug release duration is proportional to the particle size [209], to further improve the ocular therapeutic duration and bioavailability of drugs released from nanoparticles, nanoparticles are embedded in a matrix such as hydrogels or hydrogel contact lenses to form a composite drug delivery system [210-213]. In this composite system, a drug needs first to diffuse through the nanoparticle, reach the hydrogel/contact lens matrix and then diffuse through the hydrogel matrix to reach the target [214]. Therefore, the drug release duration of the composition system is longer than the nanoparticles alone and also the hydrogel/contact lens matrix alone [214, 215]. In addition, the composite system can also improve the biocompatibility of the nanoparticle carriers by hiding them within the hydrogel or contact lens matrix, to prevent the nanoparticles from migrating away from their intended target site in vivo, and to minimize the drug metabolism from the enzymes present in the tear/corneal surface [216, 217]. 
Several types of nanoparticles such as liposomes or lipid-based nanoparticles [210, 213, 218-220], micelles [221, 222], polymeric and metal nanoparticles [211, 214, 217] have been loaded in hydrogels/contact lenses to design extended-release composite systems with varying degree of success. Besides contact lenses, other composite systems including implants, fibers or in situ gels were also used to load nanoparticles [218, 223]. Drugs including lidocaine [210], ciprofloxacin [219], levofloxacin [220], CsA [221, 222], timolol [213, 217], silver [224], meloxicam [225], dexamethasone [226-228], loteprednol etabonate [214], hydrocortisone butyrate [223], and resina draconis [218] have been loaded into the nanoparticles to be delivered through the composite systems. Four different methods have been used to prepare nanoparticle-laden therapeutic contact lenses. The first most common method includes two steps: nanoparticles are first prepared and loaded with drugs at size 3$100 \mathrm{~nm}$, and then dispersed in HEMA monomer solution to form nanoparticle embedded contact lens after polymerization of HEMA monomers is initiated [211, 214, 217]. The second method is attained by a single step process with surfactants included during the polymerization process to form micellar aggregates in the polymer hydrogel matrix to incorporate hydrophobic drugs [221,222]. The third method is to load the nanoparticles into prefabricated lenses by soaking the lenses in a solution (e.g. ethanol) containing the nanoparticles [217]. The fourth method is to immobilize drug loaded nanoparticles on the surface of contact lenses [229, 230]. In this Section, we will review liposomes, lipid nanoparticles, micelles, polymeric and metal nanoparticle-based composite systems for drug delivery to the anterior segment.

\subsubsection{Liposome/Lipid nanoparticle-based composite systems-The use of} liposomes for incorporation of the drug within contact lens matrices was explored initially by Chauhan and his associates. Poly(2-hydroxyethyl methacrylate) (PHMEA) contact lenses encapsulated with lidocaine-loaded dimyristoyl phosphatidylcholine liposomes (size $\sim 20$ $\mathrm{nm}$ ) showed a sustained in vitro release of lidocaine with $65 \%$ drug release in 7 days and an initial burst of about 15-30\%, but had slightly lower transparency (80\%) compared with the blank lenses (90\%) [210]. Instead of being dispersed, Danion et al. immobilized levofloxacin loaded liposomes on the surface of commercial contact lenses (Hioxifilcon B) [220, 229]. The lenses that were surface immobilized with 2 layers of liposomes showed drug release for up to $30 \mathrm{~h}$ while those with 10 layers of liposomes released the drug for $120 \mathrm{~h}$. However, the 10 layers of liposomes decreased oxygen and carbon dioxide permeability through the lens. The liposome-laden contact lenses were found to be non-toxic to human corneal epithelial cells, reconstructed human corneas and ex vivo rabbit corneas [220, 229]. Surface entrapment of liposomes did not affect the light transmission of the contact lenses compared with the control lenses without liposomes at the maximal photopic sensitive wavelength, and, in addition, the lens with the liposome layers afforded more eye protection in the ultraviolet spectrum, compared with the control lenses [230]. Jain et al. prepared drugeluting contact lens by coating soft contact lenses with ciprofloxacin-loaded liposomes including unilamellar or multilamellar vesicles [219]. Multilamellar vesicles (mean diameter 338.32)-containing liposomes had a larger size, exhibited higher values of entrapment efficiencies ( 1.5 to 3.9 times), and showed greater sustained action than unilamellar vesicle (mean diameter $120.42 \mathrm{~nm}$ )-containing liposomes. The liposomal formulations were found to be non-toxic and also to lower the toxicity of ciprofloxacin when evaluated by 
lymphocyte toxicity assay and chick embryo inoculation. The contact lens coated with ciprofloxacin-entrapped liposomes showed much longer in vitro residence time than ciprofloxacin eye drops (24 h vs. $2-5 \mathrm{~min}$ ) [219]. Yu et al. prepared a composite system wherein timolol maleate-containing liposomes $(136 \pm 18 \mathrm{~nm})$ were dispersed into deacetylated gellan gum-based ion sensitive in situ gels [213]. The composite system showed more sustained in vitro timolol maleate release than liposome nanoparticles alone ( 24 vs. $2.5 \mathrm{~h}$ for $\sim 90 \%$ release). It also increased timolol maleate's corneal retention time (>10 min retention time vs. clear by $10 \mathrm{~min}$ ) and IOP-lowering capability ( $\sim 5 \mathrm{~h}$ vs. $\sim 3 \mathrm{~h}$ duration of action) in rabbits, compared with timolol maleate eye drops. However, no in vivo studies with timolol maleate-loaded liposomes were reported by the group [213].

Hao et al. explored the potential of a novel hybrid composite system composed of glyceryl monostearate (GMS)-based solid lipid nanoparticles embedded in poloxamer-based thermosensitive hydrogel for ophthalmic delivery using Resina Draconis as a model drug [218]. Incorporation of SLNs into the hydrogel resulted in an increase in the size of the SLNs from 150 to $450 \mathrm{~nm}$, probably due to a sheath around the SLN formed by the poloxamer polymer from the hydrogel that adsorbed on and entangled with the SLN. The size of SLN in hybrid hydrogel system was observed to be stable for 30 days of storage at room temperature. This composite system showed non-irritancy in Hen's Egg Test Chorioallantoic Membrane (HET-CAM) test. It delayed and decreased the penetration of Resina Draconis across the ex vivo albino rabbit cornea in comparison with the SLN alone (lag time 23 vs. $12 \mathrm{~min}$, and permeability coefficient $15.32 \times 10^{6}$ vs. $10.09 \times 10^{6} \mathrm{~cm} \cdot \mathrm{sec}^{-1}$ ) due to the presence of viscosity enhancer poloxamer in the composite system. Confocal microscopy study showed that coumarin C-6-labeled SLN could penetrate through the cornea and reach the inner corneal endothelial layer in $2 \mathrm{~h}$ and continuously accumulated at the endothelial layer till at least $12 \mathrm{~h}$ after instillation of the SLN-embedded hydrogel composition system into albino rabbit eyes [218].

3.7.2. Micelle-based composite systems-The use of micelles for drug incorporation in contact lens has so far demonstrated to be more promising for prolonged drug release while maintaining the desired properties of contact lenses than the use of liposomes. The above discussed liposome/lipid nanoparticles based composition systems have not been reported to show more than 5 days' drug release; while non-ionic Brij ${ }^{\circledR}$ surfactant-laden pHEMA hydrogel contact lenses developed by Chauhan and associates could sustain the release of CsA for up to at least 60 days, depending on the type and concentration of the surfactants, without compromising the mechanical and optical properties of the contact lens [221, 222]. In addition, the sustained release duration depended on drugs' properties too. For example, dexamethasone and dexamethasone 21 acetic acid derivatives could not be brought into sustained release by the same non-ionic Brij (Brij-78) surfactant-laden p-HEMA contact lenses used for the successful sustained release of CsA due to the poor partition of these molecules inside surfactant micellar aggregates [221, 222]. For anionic dexamethasone phosphate, cationic surfactant cetalkonium chloride was used to encapsulate the drug and then this was dispersed in p-HEMA contact lenses with $10 \%$ surfactant loading [226]. The in vitro release of dexamethasone phosphate from the composition system was prolonged for $50 \mathrm{~h}, 24$ times longer than the unmodified p-HEMA contact lenses. Furthermore, the 
composite system also improved the wettability and protein binding of the contact lens [226]. For dexamethasone acetate, silica shell crosslinked methoxy PEG-block-PCL micelles (rod-like morphology) were used to encapsulate the drug and then this was dispersed in poly(2-hydroxyethyl methacrylate-methacrylic acid-ethylene glycol dimethacrylate) hydrogel soft contact lenses. This composite system could sustain the release of dexamethasone acetate for up to 30 days while maintaining the optical transparency of the contact lens [227].

3.7.3. Polymeric or other nanoparticle-based composite systems-Researchers have focused on polymeric nanoparticles using biodegradable and nonbiodegradable polymers such as poly(ethylene glycol dimethacrylate) (PEGDMA), poly(propoxylated glyceryl triacylate) (PPGT), pullulan, PCL, chitosan and albumin to develop therapeutic contact lenses to treat eye diseases [211, 214, 217, 228, 231]. Jung et al. reported a p-HEMA contact lens containing timolol-covalently-bound nanoparticles (size- $3.5 \mathrm{~nm}$ ) made of EGDMA and PGT. The contact lens could slowly release timolol for 2-4 weeks due to the hydrolytic cleavage of the ester bond between timolol and the nanoparticles [211]. The timolol release was found to be first order kinetics with rate constant depending on temperature ranging from $25-100{ }^{\circ} \mathrm{C}$; the rate constant varied directly with temperature, as expected from the Arrhenius equation. Under refrigerated storage conditions, the kinetic rate constant was observed to be low, so that timolol was retained in the lens to prevent premature timolol release before the contact lens was put in. Despite these promising results, the composite system had drawbacks of decreasing the water content and oxygen permeability of the contact lens with increasing the loading of the nanoparticles and could not be used for extended wear [211]. In a similar way, Jung et al. reported that timololloaded PGT nanoparticles dispersed in extended wear silicone hydrogel contact lens could sustain the release of timolol for one month in vitro and the release kinetics depended on temperature; but negative impact on the critical properties of the lens was observed, including water content, modulus, and ion and oxygen permeability with increasing the loading of the nanoparticles in the contact lens [217]. Preliminary in vivo studies in beagle dogs demonstrated safety and efficacy of this composite system in lowering IOP for 4 days. However, no in vitro release or in vivo studies with either drug-loaded contact lenses or drug-loaded nanoparticles were reported by the group [217]. Garhwal et al. prepared ciprofloxacin-loaded core-shell nanospheres made of copolymer of pullulan and PCL; and then incorporated the nanospheres into a conventional, transparent p-HEMA contact lens for the potential treatment of bacterial keratitis [231]. Depending on their thickness, the lenses exhibited antibacterial activity for up to 3 days due to sustained release of ciprofloxacin that efficiently inhibited the proliferation of bacteria Staphylococcus aureus and Pseudomonas aeruginosa [231]. Hashemi Nasra et al. synthesized hybrid nanoparticles by crosslinking PCL (hydrophobic inner core), HEMA (hydrophobic inner shell), and PEG-diacrylate (hydrophilic outer shell) and encapsulated the anti-inflammatory drug loteprednol etabonate into the nanoparticles [214]. The drug-loaded nanoparticles were dispersed in poly(HEMA$c o-\mathrm{N}$-vinylpyrrolidone) hydrogel contact lenses. The drug release rate of the composite system $(\sim 56 \%$ in $290 \mathrm{~h})$ is slower than those of the drug-loaded nanoparticles ( $52 \%$ in 100 h) and hydrogel containing free drug $(\sim 55 \%$ in $50 \mathrm{~h})$, indicating that incorporating nanoparticles in the hydrogel further retards the drug release. The hybrid system was found 
to be nontoxic in vitro on rabbit corneal epithelial cells [214]. Behl et al. incorporated negatively charged dexamethasone drug into positively charged chitosan (low MW 20300cP) nanoparticles, and then loaded them into PHEMA based contact lenses [228]. The obtained composite system had similar optical property as the blank contact lens without the nanoparticles, and showed sustained release of dexamethasone in vitro with $~ 56 \%$ drug release in 22 days and $\sim 10 \%$ initial burst [228].

Besides the above mentioned drug-loaded nanoparticles, some other nanoparticles, such as silver nanoparticles and meloxicam nanocrystals, which directly showed therapeutic effects, were incorporated in contact lenses to fabricate therapeutic contact lenses. Bazzaz et al. reported that silver nanoparticles, loaded into a proxy for soft hydrogel contact lenses, showed excellent antimicrobial activity against $P$. aeruginosa, relative to the $S$. aureus, and exhibited the activity over a period of $6-72 \mathrm{~h}$ depending on the concentration of the silver nanoparticles and bacterial species. They envisioned that the contact lenses prepared from the silver nanoparticle-laden hydrogel material could provide enough antibacterial effect to lower the risk of microbial-related unfavorable events for lens wearers. However, no study about the effects of nanoparticle-loading on the properties of the contact lens was reported by the group [224]. Zhang et al. prepared meloxicam nanocrystals-laden contact lenses for the treatment of post-cataract endophthalmitis [225]. Aggregates of meloxicam nanocrystals at $\sim 100 \mathrm{~nm}$ were first coated with bovine serum albumin to lower the ocular irritancy of meloxicam and then dispersed in p-HEMA contact lenses to achieve 5 day release of meloxicam. The meloxicam release kinetics depended on the thickness and degree of crosslinking of the contact lenses. In vivo ocular irritation studies using the Draize test on New Zealand white albino rabbits demonstrated that the meloxicam nanocrystals-laden contact lenses were significantly less irritating to the ocular tissues when compared with the marketed drug solution alone [225]. Yavuz et al. prepared CsA-loaded PLGA 85:15 or PCL (MW 30,000-70,000 Da) nanoparticles by o/w emulsification solvent evaporation technique and then incorporated the nanoparticles into PCL implant/fiber composite system by electrospinning/implant molding method [232]. They found that CsA was present in cornea, sclera and lens even 90 days after subconjunctival implantation of the composite systems in dry-eye induced mice. The fiber composite system had better CsA tissue concentration and healing process than the implant composite system [232]. Yang et. al. also used PLGA nanoparticles (113-187 nm) to load hydrocortisone butyrate and then suspended them in a thermosensitive PLGA-PEG-PLGA gel. They used surfactants chitosan, pluronic and PVA to prepare the nanoparticles, and found that the nanoparticles made of chitosan (187 nm size and $+31 \mathrm{mV}$ zeta potential) were taken up by human corneal epithelial cells more than the nanoparticles made of PVA (164 nm size and $-20 \mathrm{mV}$ zeta potential) or pluronic (113 nm size and $-26.9 \mathrm{mV}$ zeta potential). The nanoparticle-laden gel composite system released hydrocortisone slower ( 100\% in 28-32 days, no burst) than both the drug-loaded nanoparticles ( $70 \%$ in 1 day and $\sim 80 \%$ in 16 days) and gel ( $45 \%$ in $24 \mathrm{~h}$ and $\sim 85 \%$ in 24 days). Ibrahim et al. evaluated the stability of nanoparticles made of chitosan, sodium alginate, PCL, PLA and PLGA under accelerated stability testing methods [110]. They found that the nanoparticles made of chitosan and PCL were more stability and had longer shelf life than the nanoparticles made of the other materials. They also found that when composite systems made of two types of nanoparticles dispersed in HPMC gel could 
significantly increase the ocular bioavailability of celecoxib by $\sim 4.8-29.7$-fold in comparison to the drug suspension. The chitosan-nanoparticles-laden gel increased the ocular bioavailability of celecoxib more than the PCL-nanoparticles laden gel due to the stronger mucoadhesive and penetration enhancement properties of chitosan [110].

Table 14 summarizes various nanoparticle-based composite systems and their characteristics.

\section{Safety and regulatory considerations}

Nanomaterials can exhibit altered physical or chemical properties or biological effects (dimension-dependent properties or phenomena) in comparison to their corresponding largescale materials with the same chemical composition [233-236]. Each class of nanoparticles is unique, and "FDA believes that evaluations of safety, effectiveness, public health impact, or regulatory status of nanotechnology products should consider any unique properties and behaviors that the application of nanotechnology may impart" [233]. There are three major considerations related to the products based on nanotechnology, which includes characterization, safety, and environmental impact [237]. Various essential parameters that need to be considered during the characterization of nanoparticles are: critical physical and chemical properties and their impact on quality and performance of the product, reliable and reproducible in vitro and in vivo characterization tools or techniques and their sensitivity, short/long-term stability issues in various environments, critical quality attributes, critical processing variables, overall chemistry, manufacturing, and controls (CMC), and the forms in which they were presented to the host [237]. The important parameters that need to be considered for evaluating the in vivo safety of nanoparticles are: tissue penetration/ distribution and pharmacokinetics parameters (ADME profile) of the nanomaterials compared to their large-scale counterparts, tissue retention, transient and/or permanent bioeffects in vivo, clearance mechanisms, and potential variation in effects in different tissues [238]. The major parameters that need to be considered for assessing the environmental impact of the nanomaterials are: potential implications of the releasing of these materials into the environment post use, and methodologies to detect and quantify the environmental effects [237]. In June 2014, FDA released the final guidance for industry related to nanomaterials - "Considering Whether an FDA-Regulated Product Involves the Application of Nanotechnology" [233]. Currently, FDA addresses the issues related to the safety, effectiveness, and public health impact of nanotechnology products on a case by case basis, and advises industry to consult with the agency in the early development process [233]. Therefore, any entity that plans to develop a nanoparticle product for ophthalmic applications, should follow the FDA guidelines and communicate with FDA as early as possible when it is feasible to learn the regulatory requirements and implement appropriate steps for bringing the product into the market.

\section{Summary and perspectives}

Nanoparticles have shown significant promise for effective drug delivery to the anterior segment of the eye. Over the past few years, increasing progress has been occurring in this area and can be further improved for clinical translation. Current development of 
nanoparticles for anterior eye segment drug delivery mainly focuses on the route of topical administration. Although the subconjunctival administration is well studied for implants, it is scarcely studied for nanoparticles in the literature. In addition, systemic and especially intracameral administrations were viewed as possibilities for drug delivery to the anterior eye segment. To date, there is no nanoparticle that has been designed to utilize these two routes of administration. We think that more studies on the effectiveness of subconjunctival, intracameral and systemic routes for delivering drugs to the anterior segment of the eye are needed and will be beneficial. Most of the nanoparticles for drug delivery to the anterior eye segment are evaluated in terms of their physicochemical properties, drug loading capability, in vitro drug release and toxicity, and comparatively simple in vivo tests. With the array of polymers and fabrication techniques currently available, nanoparticles can be engineered in a way that makes them safer, more stable, and easier to prepare; provide better control over drug release; and have the capability to deliver not only small molecules, but also peptides/ proteins and genes. With the choice of a wide range of biodegradable polymers that have emerged, there is an increased versatility in the formulation of nanoparticles in terms of category and function. We envision that more innovative, functional, practical and safe nanoparticles, including nanoparticle-loaded therapeutic contact lenses, will be continuously developed to effectively deliver drugs to the anterior segment of the eye. During future nanoparticle development, several key areas of study need to be addressed, including active targeting, pharmacokinetic/pharmacodynamics (PK/PD), safety and toxicology, stability, and production scale-up.

Active targeting based on the binding affinity between a targeting ligand attached on the nanoparticle surface and the targeted units of diseased tissue (receptors/antigens) has been considered as an effective approach for improving the efficacy of nanoparticle-based delivery systems [239, 240]. Since polymers can offer many reactive functional groups, active targeting to specific tissues in the anterior eye segment is expected to be developed by conjugating targeting moieties onto the polymeric nanoparticle surface in the near future. However, as the active targeting is a complex process, the following factors need to be considered thoroughly during the nanoparticle development for active targeting: targeted unit localization and expression in the diseased site, targeting ligand selection, conjugation chemistry, ligand density, nanoparticle size and architecture, accessibility of the nanoparticles to the target site, non-specific binding, and in vivo stability of the ligandconjugated nanoparticles [239, 241-244].

As nanoparticles can alter the PK/PD properties of a free drug and subsequently the therapeutic effect and toxicity of the drug [182], there is an unmet need to critically assess the PK, and local and systemic biodistribution of the nanoparticles after administration into the eye. It is also in need to study how ocular diseases affect anterior segment nanodelivery. In addition to the PK/PD studies, more focused emphasis should be given to study the acute and long-term toxicity of the nanoparticles, and the ultimate fate of the nanoparticles or their degradants after the release of the drug at the intended site. More deeply, it is important to understand and measure the effects of nanoparticulate parameters (size, charge, architecture, surface chemistry, active targeting) on the in vivo fate including clearance, and the long-term toxicity of the nanoparticles. Future studies can also be performed in evaluating the specific interactions of the nanoparticles with cells, tissues and/or human organs, and the 
mechanisms for the nanoparticles to enhance the drug bioavailability in the anterior eye segment.

Physical and chemical stability is another key aspect that needs to be considered during the pharmaceutical development of drug-nanoparticulate systems to ensure the safety, efficacy, and quality of the products $[165,245]$. Currently, there are scarce studies about the stability of nanoparticles for ocular applications. In the future development, the physical, chemical, biological, and microbiological stability of nanoparticles should be evaluated according to regulatory guidelines. Most of the reported methods for fabricating nanoparticles for ocular applications are at lab scale and not suitable for industry mass production. In the future, cost-effective technology needs to be developed to scale up nanoparticle production for clinical translation. During scale up, special consideration should also be given to the techniques for sterilizing the nanoparticles such as aseptic processing/sterile filtration or terminal sterilization by autoclaving. Besides the above discussed key areas of future studies, the evaluation of the environmental impacts of the nanomaterials needs to be carried out in the future [237]. As nanoparticles are still in their early development stage for treating diseases in the anterior segment of the eye, there is no clinical trial for nanoparticle systems yet. With the further development of nanoparticles for drug delivery and other treatments for the anterior eye segment, appropriately designed clinical trials for the nanoparticle systems are expected to be conducted in the future.

\title{
Acknowledgments
}

The work was supported by the NIH R21EY017145 and NIH R01EY023853.

\author{
Abbreviations \\ PACA poly(alkyl cyanoacrylate) \\ CsA cyclosporine A \\ IOBA-NHC spontaneously immortalized epithelial cell line from normal human \\ conjunctiva \\ HCE human corneal epithelial cells \\ FITC fluorescein isothiocyanate \\ HA hyaluronic acid \\ IOP intraocular pressure \\ CH cholesterol \\ CS-CH chlolesterol modified chitosan \\ PLA poly(lactide) \\ PLGA poly(lactide-co-glycoside) \\ PCL $\quad \operatorname{poly}(\varepsilon$-caprolactone)
}




\begin{tabular}{|c|c|}
\hline GRGDS & Gly-Arg-Gly-Asp-Ser \\
\hline RGD & Arg-Gly-Asp \\
\hline BXD & boxed molecular dynamics \\
\hline BKC & benzalkonium chloride \\
\hline SLN & solid lipid nanoparticles \\
\hline RCE & corneal epithelial cells \\
\hline GMS & glyceryl monosterate \\
\hline HET-CAM & Hen's Egg Test - Chorioallantoic Membrane \\
\hline NLC & nanosctructured lipid carriers \\
\hline PAMAM & polyamidoamine \\
\hline DPT & dendritic polyguanidilyated translocators \\
\hline PHEMA & poly(2-hydroxyethyl methacrylate) \\
\hline PEG & poly(ethylene glycol) \\
\hline EGDMA & ethylene glycol dimethacrylate \\
\hline PEGDMA & poly(ethylene glycol dimethacrylate) \\
\hline PPGT & poly(propoxylated glyceryl triacylate) \\
\hline VEGF & vascular endothelial growth \\
\hline PDI & polydispersity index \\
\hline $\boldsymbol{A U C}$ & the area under curve \\
\hline$M R T$ & mean residence time \\
\hline CS-NAC & N-acetyl-L-cysteine functionalized chitosan \\
\hline PK & pharmacokinetics \\
\hline PD & pharmacodynamics \\
\hline HEC & human corneal epithelial cells \\
\hline
\end{tabular}

\section{References}

1. Cunha-Vaz JG. The blood-ocular barriers: Past, present, and future. Documenta Ophthalmologica. 1997; 93:149-157. [PubMed: 9476613]

2. Wadhwa S, Paliwal R, Vyas SP. Nanocarriers in ocular drug delivery: An update review. Current Pharmaceutical Design. 2009; 15:2724-2750. [PubMed: 19689343]

3. Gaudana R, Ananthula HK, Parenky A, Mitra AK. Ocular drug delivery. The AAPS journal. 2010; 12:348-360. [PubMed: 20437123]

Adv Drug Deliv Rev. Author manuscript; available in PMC 2018 December 01. 
4. Bucolo C, Drago F, Salomone S. Ocular drug delivery: a clue from nanotechnology. Frontiers in pharmacology. 2012; 3:188. [PubMed: 23125835]

5. Ghate D, Edelhauser HF. Ocular drug delivery. Expert Opinion on Drug Delivery. 2006; 3:275-287. [PubMed: 16506953]

6. Lee VHL, Robinson JR. Review: Topical ocular drug delivery: Recent developments and future challenges. Journal of Ocular Pharmacology. 1986; 2:67-108. [PubMed: 3332284]

7. Urtti A. Challenges and obstacles of ocular pharmacokinetics and drug delivery. Advanced drug delivery reviews. 2006; 58:1131-1135. [PubMed: 17097758]

8. Worakul N, Robinson JR. Ocular pharmacokinetics/pharmacodynamics. European journal of pharmaceutics and biopharmaceutics. 1997; 44:71-83.

9. Klyce SD, Crosson CE. Transport processes across the rabbit corneal epithelium: a review. Current eye research. 1985; 4:323-331. [PubMed: 3893897]

10. Ahmed I, Patton TF. Importance of the noncorneal absorption route in topical ophthalmic drug delivery. Investigative Ophthalmology and Visual Science. 1985; 26:584-587. [PubMed: 3884542]

11. Watsky MA, Jablonski MM, Edelhauser HF. Comparison of conjunctival and corneal surface areas in rabbit and human. Current Eye Research. 1988; 7:483-486. [PubMed: 3409715]

12. Sasaki H, Igarashi Y, Nagano T, Nishida K, Nakamura J. Different effects of absorption promoters on corneal and conjunctival penetration of ophthalmic beta-blockers. Pharmaceutical Research. 1995; 12:1146-1150. [PubMed: 7494826]

13. Ahmed I, Gokhale RD, Shah MV, Patton Th F. Physicochemical determinants of drug diffusion across the conjunctiva, sclera, and cornea. Journal of Pharmaceutical Sciences. 1987; 76:583-586. [PubMed: 11002815]

14. Ahmed I, Gokhale RD, Shah MV, Patton TF. Physicochemical determinants of drug diffusion across the conjunctiva, sclera, and cornea. Journal of pharmaceutical sciences. 1987; 76:583-586. [PubMed: 11002815]

15. Prausnitz MR, Noonan JS. Permeability of cornea, sclera, and conjunctiva: a literature analysis for drug delivery to the eye. Journal of pharmaceutical sciences. 1998; 87:1479-1488. [PubMed: 10189253]

16. She SC, Steahly LP, Moticka EJ. Intracameral injection of allogeneic lymphocytes enhances corneal graft survival. Investigative Ophthalmology and Visual Science. 1990; 31:1950-1956. [PubMed: 2210990]

17. Lane SS, Osher RH, Masket S, Belani S. Evaluation of the safety of prophylactic intracameral moxifloxacin in cataract surgery. J Cataract Refract Surg. 2008; 34:1451-1459. [PubMed: 18721703]

18. Braga-Mele R, Chang DF, Henderson BA, Mamalis N, Talley-Rostov A, Vasavada A, Comm ACC. Intracameral antibiotics: Safety, efficacy, and preparation. J Cataract Refract Surg. 2014; 40:21342142. [PubMed: 25465691]

19. Ho JW, Afshari NA. Advances in cataract surgery: preserving the corneal endothelium. Current Opinion in Ophthalmology. 2015; 26:22-27. [PubMed: 25415300]

20. Vazirani J, Basu S. Role of topical, subconjunctival, intracameral, and irrigative antibiotics in cataract surgery. Current Opinion in Ophthalmology. 2013; 24:60-65. [PubMed: 23080014]

21. Qureshi K, Kashani S, Kelly SP. Intracameral bevacizumab for rubeotic glaucoma secondary to retinal vein occlusion. International ophthalmology. 2009; 29:537-539. [PubMed: 18791852]

22. Maris PJ Jr, Correnti AJ, Donnenfeld ED. Intracameral triamcinolone acetonide as treatment for endothelial allograft rejection after penetrating keratoplasty. Cornea. 2008; 27:847-850. [PubMed: 18650676]

23. Shsrma A, Kuppermann B. New and in-development treatments for diabetic macular edema. Retinal physician. 2008; 5:20-30.

24. Barar J, Javadzadeh AR, Omidi Y. Ocular novel drug delivery: impacts of membranes and barriers. Expert opinion on drug delivery. 2008; 5:567-581. [PubMed: 18491982]

25. Fresta M, Fontana G, Bucolo C, Cavallaro G, Giammona G, Puglisi G. Ocular tolerability and in vivo bioavailability of poly (ethylene glycol)(PEG)- coated polyethyl- 2-cyanoacrylate nanosphere- encapsulated acyclovir. Journal of pharmaceutical sciences. 2001; 90:288-297. [PubMed: 11170022]

Adv Drug Deliv Rev. Author manuscript; available in PMC 2018 December 01. 
26. Peng CC, Burke MT, Carbia BE, Plummer C, Chauhan A. Extended drug delivery by contact lenses for glaucoma therapy. Journal of controlled release. 2012; 162:152-158. [PubMed: 22721817]

27. El-Kamel A. In vitro and in vivo evaluation of Pluronic F127-based ocular delivery system for timolol maleate. International journal of pharmaceutics. 2002; 241:47-55. [PubMed: 12086720]

28. Nagarwal RC, Kumar R, Pandit J. Chitosan coated sodium alginate-chitosan nanoparticles loaded with 5-FU for ocular delivery: in vitro characterization and in vivo study in rabbit eye. European Journal of Pharmaceutical Sciences. 2012; 47:678-685. [PubMed: 22922098]

29. Li R, Chow A, Yip M, Raymond K. Basic concepts of bioavailability. HONG KONG MEDICAL JOURNAL. 1995; 1:63-63.

30. Kakkar S, Karuppayil SM, Raut JS, Giansanti F, Papucci L, Schiavone N, Kaur IP. Lipidpolyethylene glycol based nano-ocular formulation of ketoconazole. International journal of pharmaceutics. 2015; 495:276-289. [PubMed: 26325312]

31. Toutain P-L, BOUSQUET-MÉLOU A. Bioavailability and its assessment. Journal of veterinary pharmacology and therapeutics. 2004; 27:455-466. [PubMed: 15601440]

32. Nagarwal RC, Kant S, Singh P, Maiti P, Pandit J. Polymeric nanoparticulate system: a potential approach for ocular drug delivery. Journal of Controlled Release. 2009; 136:2-13. [PubMed: 19331856]

33. Ludwig A. The use of mucoadhesive polymers in ocular drug delivery. Advanced Drug Delivery Reviews. 2005; 57:1595-1639. [PubMed: 16198021]

34. Keister J, Cooper E, Missel P, Lang J, Hager D. Limits on optimizing ocular drug delivery. Journal of pharmaceutical sciences. 1991; 80:50-53. [PubMed: 2013850]

35. Sahoo SK, Dilnawaz F, Krishnakumar S. Nanotechnology in ocular drug delivery. Drug discovery today. 2008; 13:144-151. [PubMed: 18275912]

36. Davies NM. Biopharmaceutical considerations in topical ocular drug delivery. Clinical and experimental pharmacology and physiology. 2000; 27:558-562. [PubMed: 10874518]

37. Barbu E, Verestiuc L, Nevell TG, Tsibouklis J. Polymeric materials for ophthalmic drug delivery: trends and perspectives. Journal of Materials Chemistry. 2006; 16:3439-3443.

38. Sahoo SK, Dilnawaz F, Krishnakumar S. Nanotechnology in ocular drug delivery. Drug Discovery Today. 2008; 13:144-151. [PubMed: 18275912]

39. Mudgil M, Gupta N, Nagpal M, Pawar P. Nanotechnology: A new approach for ocular drug delivery system. International Journal of Pharmacy and Pharmaceutical Sciences. 2012; 4:105112 .

40. Goyal G, Garg T, Rath G, Goyal AK. Current Nanotechnological Strategies for Treating Glaucoma. Critical Reviews in Therapeutic Drug Carrier Systems. 2014; 31:365-405. [PubMed: 25271557]

41. Kambhampati SP, Kannan RM. Dendrimer Nanoparticles for Ocular Drug Delivery. Journal of Ocular Pharmacology and Therapeutics. 2013; 29:151-165. [PubMed: 23410062]

42. Suresh PK, Sah AK. Nanocarriers for ocular delivery for possible benefits in the treatment of anterior uveitis: focus on current paradigms and future directions. Expert Opinion on Drug Delivery. 2014; 11:1747-1768. [PubMed: 25007007]

43. Yavuz B, Pehlivan SB, Unlu N. Dendrimeric Systems and Their Applications in Ocular Drug Delivery. Scientific World Journal. 2013

44. Zhou HY, Hao JL, Wang S, Zheng Y, Zhang WS. Nanoparticles in the ocular drug delivery. International Journal of Ophthalmology. 2013; 6:390-396. [PubMed: 23826539]

45. Diebold Y, Calonge M. Applications of nanoparticles in ophthalmology. Progress in retinal and eye research. 2010; 29:596-609. [PubMed: 20826225]

46. Cai X, Conley S, Naash M. Nanoparticle applications in ocular gene therapy. Vision research. 2008; 48:319-324. [PubMed: 17825344]

47. ThassuD, , ChaderGJ. Ocular drug delivery systems: barriers and application of nanoparticulate systemsCRC Press; 2012

48. Hillaireau H, Couvreur P. Nanocarriers' entry into the cell: relevance to drug delivery. Cellular and Molecular Life Sciences. 2009; 66:2873-2896. [PubMed: 19499185]

Adv Drug Deliv Rev. Author manuscript; available in PMC 2018 December 01. 
49. Zhang Y, Chen J, Zhang Y, Pan Y, Zhao J, Ren L, Liao M, Hu Z, Kong L, Wang J. A novel PEGylation of chitosan nanoparticles for gene delivery. Biotechnology and applied biochemistry. 2007; 46:197-204. [PubMed: 17147512]

50. Walsh M, Tangney M, O’Neill M, Larkin J, Soden D, McKenna SL, Darcy R, O'sullivan G, O'Driscoll C. Evaluation of cellular uptake and gene transfer efficiency of pegylated poly-L-lysine compacted DNA: implications for cancer gene therapy. Molecular pharmaceutics. 2006; 3:644653. [PubMed: 17140252]

51. Tong YC, Chang SF, Liu CY, Kao WWY, Huang CH, Liaw J. Eye drop delivery of nanopolymeric micelle formulated genes with cornea- specific promoters. The journal of gene medicine. 2007; 9:956-966. [PubMed: 17724775]

52. Contreras-Ruiz L, de la Fuente M, Párraga JE, López-García A, Fernández I, Seijo B, Sánchez A, Calonge M, Diebold Y. Intracellular trafficking of hyaluronic acid-chitosan oligomer-based nanoparticles in cultured human ocular surface cells. 2011

53. De la Fuente M, Seijo B, Alonso M. Bioadhesive hyaluronan-chitosan nanoparticles can transport genes across the ocular mucosa and transfect ocular tissue. Gene therapy. 2008; 15:668-676. [PubMed: 18305575]

54. Zimmer A, Kreuter J, Robinson JR. Studies on the transport pathway of PBCA nanoparticles in ocular tissues. Journal of Microencapsulation. 1991; 8:497-504. [PubMed: 1798020]

55. Agarwal P, Rupenthal ID. In vitro and ex vivo corneal penetration and absorption models. Drug delivery and translational research. 2016; 6:634-647. [PubMed: 26762419]

56. Hiratani H, Alvarez-Lorenzo C. Timolol uptake and release by imprinted soft contact lenses made of N, N-diethylacrylamide and methacrylic acid. Journal of Controlled Release. 2002; 83:223-230. [PubMed: 12363448]

57. Peng CC, Chauhan A. Extended cyclosporine delivery by silicone-hydrogel contact lenses. Journal of Controlled Release. 2011; 154:267-274. [PubMed: 21745507]

58. Yuan X, Marcano DC, Shin CS, Hua X, Isenhart LC, Pflugfelder SC, Acharya G. Ocular drug delivery nanowafer with enhanced therapeutic efficacy. ACS nano. 2015; 9:1749-1758. [PubMed: 25585134]

59. Coursey TG, Henriksson JT, Marcano DC, Shin CS, Isenhart LC, Ahmed F, De Paiva CS, Pflugfelder SC, Acharya G. Dexamethasone nanowafer as an effective therapy for dry eye disease. Journal of Controlled Release. 2015; 213:168-174. [PubMed: 26184051]

60. Shin C, Marcano D, Henriksson J, Acharya G, Pflugfelder SC. Nanowafer Drug Delivery for Restoration of Healthy Ocular Surface in Dry Eye Condition. Investigative Ophthalmology \& Visual Science. 2015; 56:321-321.

61. Acharya G. The dexamethasone nanowafer: A novel method of drug delivery for dry eye disease. 2015

62. Vauthier C, Dubernet C, Fattal E, Pinto-Alphandary H, Couvreur P. Poly(alkylcyanoacrylates) as biodegradable materials for biomedical applications. Advanced Drug Delivery Reviews. 2003; 55:519-548. [PubMed: 12706049]

63. Wood RW, Li VHK, Kreuter J, Robinson JR. Ocular disposition of poly-hexyl-2-cyano[314C] acrylate nanoparticles in the albino rabbit. Int J Pharm. 1985; 23:175-183.

64. Harmia T, Kreuter J, Speiser P. Enhancement of the myotic response of rabbits with pilocarpineloaded polybutylcyanoacrylate nanoparticles. Int J Pharm. 1986; 33:187-193.

65. Fitzgerald P, Hadgraft J, Kreuter J, Wilson CG. A $\gamma$-scintigraphic evaluation of microparticulate ophthalmic delivery systems: liposomes and nanoparticles. Int J Pharm. 1987; 40:81-84.

66. Diepold R, Kreuter J, Guggenbuhl P, Robinson JR. Distribution of poly-hexyl-2-cyano-[314C] acrylate nanoparticles in healthy and chronically inflamed rabbit eyes. Int J Pharm. 1989; 54:149-153.

67. Marchal-Heussler L, Maincent P, Hoffman M, Spittler J, Couvreur P. Antiglaucomatous activity of betaxolol chlorhydrate sorbed onto different isobutylcyanoacrylate nanoparticle preparations. Int $\mathrm{J}$ Pharm. 1990; 58:115-122.

68. Losa C, Calvo P, Castro E, Vila-Jato JL, Alonso MJ. Improvement of ocular penetration of amikacin sulphate by association to poly(butylcyanoacrylate) nanoparticles. Journal of Pharmacy and Pharmacology. 1991; 43:548-552. [PubMed: 1681069] 
69. Mann A, Kiefer M, Leuenberger H. Ocular tolerability and in vivo bioavailability of poly(ethylene glycol) (PEG)-coated polyethyl-2-cyanoacrylate nanosphere-encapsulated acyclovir. Journal of Pharmaceutical Sciences. 2001; 90:288-297. [PubMed: 11170022]

70. Lee JW, Park JH, Robinson JR. Bioadhesive-based dosage forms: The next generation. Journal of Pharmaceutical Sciences. 2000; 89:850-866. [PubMed: 10861586]

71. Lemarchand C, Gref R, Couvreur P. Polysaccharide-decorated nanoparticles. European Journal of Pharmaceutics and Biopharmaceutics. 2004; 58:327-341. [PubMed: 15296959]

72. Robinson JR, Mlynek GM. Bioadhesive and phase-change polymers for ocular drug delivery. Advanced Drug Delivery Reviews. 1995; 16:45-50.

73. Nemoto E, Ueda H, Akimoto M, Natsume H, Morimoto Y. Ability of poly-L-arginine to enhance drug absorption into aqueous humor and vitreous body after instillation in rabbits. Biological and Pharmaceutical Bulletin. 2007; 30:1768-1772. [PubMed: 17827737]

74. Dodane V, Amin Khan M, Merwin JR. Effect of chitosan on epithelial permeability and structure. International Journal of Pharmaceutics. 1999; 182:21-32. [PubMed: 10332071]

75. Majumdar S, Hippalgaonkar K, Repka MA. Effect of chitosan, benzalkonium chloride and ethylenediaminetetraacetic acid on permeation of acyclovir across isolated rabbit cornea. Int J Pharm. 2008; 348:175-178. [PubMed: 17897799]

76. Zambito Y, Zaino C, Di Colo G. Effects of N-trimethylchitosan on transcellular and paracellular transcorneal drug transport. European Journal of Pharmaceutics and Biopharmaceutics. 2006; 64:16-25. [PubMed: 16527472]

77. Bernkop-Schnürch A. Thiomers: A new generation of mucoadhesive polymers. Advanced Drug Delivery Reviews. 2005; 57:1569-1582. [PubMed: 16176846]

78. Lang JC. Ocular drug delivery conventional ocular formulations. Advanced Drug Delivery Reviews. 1995; 16:39-43.

79. Felt O, Furrer P, Mayer JM, Plazonnet B, Buri P, Gurny R. Topical use of chitosan in ophthalmology: tolerance assessment and evaluation of precorneal retention. Int J Pharm. 1999; 180:185-193. [PubMed: 10370189]

80. de Salamanca AE, Diebold Y, Calonge M, García-Vazquez C, Callejo S, Vila A, Alonso MJ. Chitosan Nanoparticles as a Potential Drug Delivery System for the Ocular Surface: Toxicity, Uptake Mechanism and In Vivo Tolerance. Invest Ophthalmol Vis Sci. 2006; 47:1416-1425. [PubMed: 16565375]

81. HiranoSHS, , AkiyamaI, , NonakaI. Chitosan: a biocompatible material for oral and intravenous administrationPlenum Press; New York: 1990

82. Artursson P, Lindmark T, Davis SS, Illum L. Effect of chitosan on the permeability of monolayers of intestinal epithelial cells (Caco-2). Pharm Res. 1994; 11:1358-1361. [PubMed: 7816770]

83. Takeuchi H, Yamamoto H, Niwa T, Hino T, Kawashima Y. Enteral absorption of insulin in rats from mucoadhesive Chitosan-Coated liposomes. Pharm Res. 1996; 13:896-901. [PubMed: 8792429]

84. Liu Z, Jiao Y, Wang Y, Zhou C, Zhang Z. Polysaccharides-based nanoparticles as drug delivery systems. Advanced Drug Delivery Reviews. 2008; 60:1650-1662. [PubMed: 18848591]

85. Alonso MJ, Sánchez A. The potential of chitosan in ocular drug delivery. Journal of Pharmacy and Pharmacology. 2003; 55:1451-1463. [PubMed: 14713355]

86. Agnihotri SA, Mallikarjuna NN, Aminabhavi TM. Recent advances on chitosan-based micro- and nanoparticles in drug delivery. Journal of Controlled Release. 2004; 100:5-28. [PubMed: 15491807]

87. Nagpal K, Singh SK, Mishra DN. Chitosan Nanoparticles: A Promising System in Novel Drug Delivery. Chemical and Pharmaceutical Bulletin. 2010; 58:1423-1430. [PubMed: 21048331]

88. Stefania Racovita SV, Popa Marcel, Luca Cornelia. Polysaccharides based on micro- and nanoparticles obtained by ionic gelation and their applications as drug delivery systems. Revue Roumaine de Chimie. 2009; 59:709-718.

89. Costa J, Silva N, Sarmento B, Pintado M. Potential chitosan-coated alginate nanoparticles for ocular delivery of daptomycin. European Journal of Clinical Microbiology \& Infectious Diseases. 2015; 34:1255-1262. [PubMed: 25754770] 
90. De Campos AM, Sanchez A, Alonso MJ. Chitosan nanoparticles: a new vehicle for the improvement of the delivery of drugs to the ocular surface. Application to cyclosporin A. Int J Pharm. 2001; 224:159-168. [PubMed: 11472825]

91. Papadimitriou S, Bikiaris D, Avgoustakis K, Karavas E, Georgarakis M. Chitosan nanoparticles loaded with dorzolamide and pramipexole. Carbohydrate Polymers. 2008; 73:44-54.

92. Katiyar S, Pandit J, Mondal RS, Mishra AK, Chuttani K, Aqil M, Ali A, Sultana Y. In situ gelling dorzolamide loaded chitosan nanoparticles for the treatment of glaucoma. Carbohydrate Polymers. 2014; 102:117-124. [PubMed: 24507263]

93. Rajendran NRNN, Kumar RS, Selvaraj S. Acyclovir-loaded chitosan nanoparticles for ocular delivery. Asian J Pharm. 2010; 4:220-226.

94. Nagarwal RC, Nath Singh P, Kant S, Maiti P, Pandit JK. Chitosan Nanoparticles of 5-Fluorouracil for Ophthalmic Delivery: Characterization, characterization, in-vitro and in-vivo study. Chemical and Pharmaceutical Bulletin. 2011; 59:272-278. [PubMed: 21297311]

95. Motwani SK, Chopra S, Talegaonkar S, Kohli K, Ahmad FJ, Khar RK. Chitosan-sodium alginate nanoparticles as submicroscopic reservoirs for ocular delivery: formulation, optimisation and in vitro characterisation. European Journal of Pharmaceutics and Biopharmaceutics. 2008; 68:513525. [PubMed: 17983737]

96. Attia Shafie M, Mohammed Fayek H. Formulation and Evaluation of Betamethasone Sodium Phosphate Loaded Nanoparticles for Ophthalmic Delivery. J Clin Exp Ophthalmol. 2013; 4:2.

97. de la Fuente M, Seijo B, Alonso MJ. Novel hyaluronic acid-chitosan nanoparticles for ocular gene therapy. Invest Ophthalmol Vis Sci. 2008; 49:2016-2024. [PubMed: 18436835]

98. Kao HJ, Lo YL, Lin HR, Yu SP. Characterization of pilocarpine-loaded chitosan/Carbopol nanoparticles. Journal of Pharmacy and Pharmacology. 2006; 58:179-186. [PubMed: 16451745]

99. Mahmoud AA, El-Feky GS, Kamel R, Awad GEA. Chitosan/sulfobutylether-beta-cyclodextrin nanoparticles as a potential approach for ocular drug delivery. Int J Pharm. 2011; 413:229-236. [PubMed: 21540097]

100. Bhatta RS, Chandasana H, Chhonker YS, Rathi C, Kumar D, Mitra K, Shukla PK. Mucoadhesive nanoparticles for prolonged ocular delivery of natamycin: In vitro and pharmacokinetics studies. International Journal of Pharmaceutics. 2012; 432:105-112. [PubMed: 22569234]

101. Chhonker YS, Prasad YD, Chandasana H, Vishvkarma A, Mitra K, Shukla PK, Bhatta RS. Amphotericin-B entrapped lecithin/chitosan nanoparticles for prolonged ocular application. International journal of biological macromolecules. 2014

102. Ibrahim MM, Abd-Elgawad AEH, Soliman OAE, Jablonski MM. Natural bioadhesive biodegradable nanoparticles-based topical ophthalmic formulations for sustained celecoxib release: In vitro Study. journal of Pharmaceutical Technology and Drug Research. 2013; 2:7.

103. Wadhwa S, Paliwal R, Paliwal SR, Vyas SP. Hyaluronic acid modified chitosan nanoparticles for effective management of glaucoma: development, characterization, and evaluation. Journal of Drug Targeting. 2010; 18:292-302. [PubMed: 19943753]

104. Sharma R, Ahuja M, Kaur H. Thiolated pectin nanoparticles: Preparation, characterization and ex vivo corneal permeation study. Carbohydr Polym. 2012; 87:1606-1610.

105. Yadav M, Ahuja M. Preparation and evaluation of nanoparticles of gum cordia, an anionic polysaccharide for ophthalmic delivery. Carbohydrate Polymers. 2010; 81:871-877.

106. Horvát G, Budai-Szúcs M, Berkó S, Szabó-Révész P, Soós J, Facskó A, Maroda M, Mori M, Sandri G, Bonferoni MC. Comparative study of nanosized cross-linked sodium-, linear sodiumand zinc-hyaluronate as potential ocular mucoadhesive drug delivery systems. International journal of pharmaceutics. 2015; 494:321-328. [PubMed: 26319587]

107. Kaur H, Ahuja M, Kumar S, Dilbaghi N. Carboxymethyl tamarind kernel polysaccharide nanoparticles for ophthalmic drug delivery. Int J Biol Macromol. 2012; 50:833-839. [PubMed: 22138451]

108. Lehr CM, Bouwstra JA, Schacht EH, Junginger HE. In vitro evaluation of mucoadhesive properties of chitosan and some other natural polymers. Int J Pharm. 1992; 78:43-48.

109. Hassan EE, Gallo JM. A simple rheological method for the in vitro assessment of mucin-polymer bioadhesive bond strength. Pharm Res. 1990; 7:491-495. [PubMed: 1694990] 
110. Ibrahim MM, Abd-Elgawad AEH, Soliman OAE, Jablonski MM. Stability and Ocular Pharmacokinetics of Celecoxib-Loaded Nanoparticles Topical Ophthalmic Formulations. Journal of pharmaceutical sciences. 2016; 105:3691-3701. [PubMed: 27789031]

111. De Campos AM, Sánchez A, Alonso MJ. Chitosan nanoparticles: A new vehicle for the improvement of the delivery of drugs to the ocular surface. Application to cyclosporin A. International Journal of Pharmaceutics. 2001; 224:159-168. [PubMed: 11472825]

112. De Campos AM, Diebold Y, Carvalho ELS, Sánchez A, Alonso MJ. Chitosan nanoparticles as new ocular drug delivery systems: In vitro stability, in vivo fate, and cellular toxicity. Pharm Res. 2004; 21:803-810. [PubMed: 15180338]

113. Ali J, Bhatnagar A, Kumar N, Ali A. Chitosan nanoparticles amplify the ocular hypotensive effect of cateolol in rabbits. International journal of biological macromolecules. 2014; 65:479-491. [PubMed: 24530326]

114. Fathalla ZM, Khaled KA, Hussein AK, Alany RG, Vangala A. Formulation and corneal permeation of ketorolac tromethamine-loaded chitosan nanoparticles. Drug Development and Industrial Pharmacy. 2016; 42:514-524. [PubMed: 26407208]

115. de la Fuente M, Raviña M, Paolicelli P, Sanchez A, Seijo B, Alonso MJ. Chitosan-based nanostructures: A delivery platform for ocular therapeutics. Advanced Drug Delivery Reviews. 2010; 62:100-117. [PubMed: 19958805]

116. Kao HJ, Lin HR, Lo YL, Yu SP. Characterization of pilocarpine-loaded chitosan/Carbopol nanoparticles. Journal of Pharmacy and Pharmacology. 2006; 58:179-186. [PubMed: 16451745]

117. Chaiyasan W, Srinivas SP, Tiyaboonchai W. Mucoadhesive Chitosan-Dextran Sulfate Nanoparticles for Sustained Drug Delivery to the Ocular Surface. Journal of Ocular Pharmacology and Therapeutics. 2013; 29:200-207. [PubMed: 23356788]

118. YuanX-b, LiH, , YuanY-b. Preparation of cholesterol-modified chitosan self-aggregated nanoparticles for delivery of drugs to ocular surface. Carbohydrate Polymers. 2006; 65:337-345.

119. Maestrelli F, Garcia-Fuentes M, Mura P, Alonso MJ. A new drug nanocarrier consisting of chitosan and hydoxypropylcyclodextrin. European Journal of Pharmaceutics and Biopharmaceutics. 2006; 63:79-86. [PubMed: 16527473]

120. Sonvico F, Cagnani A, Rossi A, Motta S, Di Bari M, Cavatorta F, Alonso M, Deriu A, Colombo P. Formation of self-organized nanoparticles by lecithin/chitosan ionic interaction. International journal of pharmaceutics. 2006; 324:67-73. [PubMed: 16973314]

121. Zhu X, Su M, Tang S, Wang L, Liang X, Meng F, Hong Y, Xu Z. Synthesis of thiolated chitosan and preparation nanoparticles with sodium alginate for ocular drug delivery. Molecular Vision. 2012; 18:1973-1982. [PubMed: 22876124]

122. Contreras-Ruiz L, De La Fuente M, García-Vázquez C, Sáez V, Seijo B, Alonso MJ, Calonge M, Diebold Y. Ocular tolerance to a topical formulation of hyaluronic acid and chitosan-based nanoparticles. Cornea. 2010; 29:550-558. [PubMed: 20335805]

123. De Campos AM, Sánchez A, Alonso MaJ. Chitosan nanoparticles: a new vehicle for the improvement of the delivery of drugs to the ocular surface. Application to cyclosporin A. International journal of pharmaceutics. 2001; 224:159-168. [PubMed: 11472825]

124. Yuan XB, Yuan YB, Jiang W, Liu J, Tian EJ, Shun HM, Huang DH, Yuan XY, Li H, Sheng J. Preparation of rapamycin-loaded chitosan/PLA nanoparticles for immunosuppression in corneal transplantation. Int J Pharm. 2008; 349:241-248. [PubMed: 17919859]

125. Chaiyasan W, Praputbut S, Kompella UB, Srinivas SP, Tiyaboonchai W. Penetration of mucoadhesive chitosan-dextran sulfate nanoparticles into the porcine cornea. Colloids and Surfaces B: Biointerfaces. 2017; 149:288-296. [PubMed: 27771493]

126. Szejtli J. Introduction and general overview of cyclodextrin chemistry. Chemical Reviews. 1998; 98:1743-1753. [PubMed: 11848947]

127. Hirayama F, Uekama K. Cyclodextrin-based controlled drug release system. Adv Drug Deliv Rev. 1999; 36:125-141. [PubMed: 10837712]

128. Ogunjimi AT, Melo SM, Vargas-Rechia CG, Emery FS, Lopez RF. Hydrophilic polymeric nanoparticles prepared from Delonix galactomannan with low cytotoxicity for ocular drug delivery. Carbohydrate Polymers. 2017; 157:1065-1075. [PubMed: 27987808] 
129. Barbault-Foucher S, Gref R, Russo P, Guechot J, Bochot A. Design of poly-e-caprolactone nanospheres coated with bioadhesive hyaluronic acid for ocular delivery. Journal of Controlled Release. 2002; 83:365-375. [PubMed: 12387945]

130. Yoncheva K, Vandervoort J, Ludwig A. Development of mucoadhesive poly(lactide-co-glycolide) nanoparticles for ocular application. Pharmaceutical Development and Technology. 2011; 16:2935. [PubMed: 20038266]

131. De Campos AM, Sánchez A, Gref R, Calvo P, Alonso MJ. The effect of a PEG versus a chitosan coating on the interaction of drug colloidal carriers with the ocular mucosa. European Journal of Pharmaceutical Sciences. 2003; 20:73-81. [PubMed: 13678795]

132. Araújo J, Vega E, Lopes C, Egea MA, Garcia ML, Souto EB. Effect of polymer viscosity on physicochemical properties and ocular tolerance of FB-loaded PLGA nanospheres. Colloids and Surfaces B: Biointerfaces. 2009; 72:48-56. [PubMed: 19403277]

133. Giannavola C, Bucolo C, Maltese A, Paolino D, Vandelli MA, Puglisi G, Lee VHL, Fresta M. Influence of preparation conditions on acyclovir-loaded poly-d,l-lactic acid nanospheres and effect of PEG coating on ocular drug bioavailability. Pharmaceutical Research. 2003; 20:584590. [PubMed: 12739765]

134. Vega E, Gamisans F, García ML, Chauvet A, Lacoulonche F, Egea MA. PLGA nanospheres for the ocular delivery of flurbiprofen: Drug release and interactions. Journal of Pharmaceutical Sciences. 2008; 97:5306-5317. [PubMed: 18425815]

135. Ibrahim MM, Abd-Elgawad AH, Soliman OA, Jablonski MM. Novel Topical Ophthalmic Formulations for Management of Glaucoma. Pharmaceutical Research. 2013; 30:2818-2831. [PubMed: 23771565]

136. Calvo P, Vila-Jato JL, Alonso MJ. Evaluation of cationic polymer-coated nanocapsules as ocular drug carriers. International Journal of Pharmaceutics. 1997; 153:41-50.

137. Gupta H, Aqil M, Khar RK, Ali A, Bhatnagar A, Mittal G. Sparfloxacin-loaded PLGA nanoparticles for sustained ocular drug delivery, Nanomedicine: Nanotechnology. Biology, and Medicine. 2010; 6:324-333.

138. Chandasana H, Prasad YD, Chhonker YS, Chaitanya TK, Mishra NN, Mitra K, Shukla PK, Bhatta RS. Corneal targeted nanoparticles for sustained natamycin delivery and their PK/PD indices: An approach to reduce dose and dosing frequency. International journal of pharmaceutics. 2014; 477:317-325. [PubMed: 25455776]

139. Aksungur P, Demirbilek M, Denkbaş EB, Vandervoort J, Ludwig A, Ünlü N. Development and characterization of Cyclosporine A loaded nanoparticles for ocular drug delivery: Cellular toxicity, uptake, and kinetic studies. Journal of Controlled Release. 2011; 151:286-294. [PubMed: 21241752]

140. Calvo P, Thomas C, Alonso MJ, Vila-Jato JL, Robinson JR. Study of the mechanism of interaction of poly( $\varepsilon$-caprolactone) nanocapsules with the cornea by confocal laser scanning microscopy. Int J Pharm. 1994; 103:283-291.

141. Qaddoumi MG, Ueda H, Yang J, Davda J, Labhasetwar V, Lee VHL. The characteristics and mechanisms of uptake of PLGA nanoparticles in rabbit conjunctival epithelial cell layers. Pharmaceutical Research. 2004; 21:641-648. [PubMed: 15139521]

142. Agnihotri SM, Vavia PR. Diclofenac-loaded biopolymeric nanosuspensions for ophthalmic application. Nanomedicine: nanotechnology, biology and medicine. 2009; 5:90-95.

143. Lu J, Shi M, Shoichet MS. Click chemistry functionalized polymeric nanoparticles target corneal epithelial cells through RGD-cell surface receptors. Bioconjugate Chemistry. 2009; 20:87-94. [PubMed: 19099361]

144. Jithan V, Uday B. In vitro delivery of nano-and micro-particles to human retinal pigment epithelial (ARPE-19) cells. Drug Dev Deliv. 2002; 2:50-57.

145. Losa C, Marchal-Heussler L, Orallo F, Vila Jato JL, Alonso MJ. Design of new formulations for topical ocular administration: Polymeric nanocapsules containing metipranolol. Pharmaceutical Research. 1993; 10:80-87. [PubMed: 8094245]

146. Jain GK, Pathan SA, Akhter S, Jayabalan N, Talegaonkar S, Khar RK, Ahmad FJ. Microscopic and spectroscopic evaluation of novel PLGA-chitosan Nanoplexes as an ocular delivery system. Colloids and Surfaces B: Biointerfaces. 2011; 82:397-403. [PubMed: 20940097] 
147. Yenice I, Mocan MC, Palaska E, Bochot A, Bilensoy E, Vural I, Irkeç M, Atilla Hincal A. Hyaluronic acid coated poly- $\varepsilon$-caprolactone nanospheres deliver high concentrations of cyclosporine A into the cornea. Experimental Eye Research. 2008; 87:162-167. [PubMed: 18675411]

148. Liu A, Peng S, Soo JC, Kuang M, Chen P, Duan H. Quantum dots with phenylboronic acid tags for specific labeling of sialic acids on living cells. Analytical chemistry. 2010; 83:1124-1130. [PubMed: 21182248]

149. Matsumoto A, Sato N, Kataoka K, Miyahara Y. Noninvasive sialic acid detection at cell membrane by using phenylboronic acid modified self-assembled monolayer gold electrode. Journal of the American Chemical Society. 2009; 131:12022-12023. [PubMed: 19663494]

150. Liu S, Chang CN, Verma MS, Hileeto D, Muntz A, Stahl U, Woods J, Jones LW, Gu FX. Phenylboronic acid modified mucoadhesive nanoparticle drug carriers facilitate weekly treatment of experimentallyinduced dry eye syndrome. Nano Research. 2015; 8:621-635.

151. Bucolo C, Maltese A, Puglisi G, Pignatello R. Enhanced ocular anti-inflammatory activity of ibuprofen carried by an eudragit RS100® nanoparticle suspension. Ophthalmic Research. 2002; 34:319-323. [PubMed: 12381895]

152. Pignatello R, Bucolo C, Ferrara P, Maltese A, Puleo A, Puglisi G. Eudragit RS100® nanosuspensions for the ophthalmic controlled delivery of ibuprofen. European Journal of Pharmaceutical Sciences. 2002; 16:53-61. [PubMed: 12113891]

153. Bucolo C, Maltese A, Maugeri F, Busà B, Puglisi G, Pignatello R. Eudragit RL100 nanoparticle system for the ophthalmic delivery of cloricromene. Journal of Pharmacy and Pharmacology. 2004; 56:841-846. [PubMed: 15233861]

154. Pignatello R, Ricupero N, Bucolo C, Maugeri F, Maltese A, Puglisi G. Preparation and characterization of eudragit retard nanosuspensions for the ocular delivery of cloricromene. AAPS PharmSciTech [electronic resource]. 2006; 7

155. Das S, Suresh PK, Desmukh R. Design of Eudragit RL 100 nanoparticles by nanoprecipitation method for ocular drug delivery, Nanomedicine: Nanotechnology. Biology, and Medicine. 2010; 6:318-323.

156. Ibrahim HK, El-Leithy IS, Makky AA. Mucoadhesive nanoparticles as carrier systems for prolonged ocular delivery of gatifloxacin/prednisolone bitherapy. Molecular Pharmaceutics. 2010; 7:576-585. [PubMed: 20163167]

157. Mandal B, Alexander KS, Riga AT. Sulfacetamide loaded eudragit RL100 nanosuspension with potential for ocular delivery. Journal of Pharmacy and Pharmaceutical Sciences. 2010; 13:510 523. [PubMed: 21486528]

158. Zimmer AK, Chetoni P, Saettone MF, Zerbe H, Kreuter J. Evaluation of pilocarpine-loaded albumin particles as controlled drug delivery systems for the eye. II. Co-administration with bioadhesive and viscous polymers. Journal of Controlled Release. 1995; 33:31-46.

159. Zimmer AK, Maincent P, Thouvenot P, Kreuter J. Hydrocortisone delivery to healthy and inflamed eyes using a micellar polysorbate 80 solution or albumin nanoparticles. International Journal of Pharmaceutics. 1994; 110:211-222.

160. Zimmer AK, Zerbe H, Kreuter J. Evaluation of pilocarpine-loaded albumin particles as drug delivery systems for controlled delivery in the eye. I. In vitro and in vivo characterisation. Journal of Controlled Release. 1994; 32:57-70.

161. Das S, Suresh PK. Nanosuspension: A new vehicle for the improvement of the delivery of drugs to the ocular surface. Application to amphotericin B. Nanomedicine: Nanotechnology, Biology, and Medicine. 2011; 7:242-247.

162. Pignatello R, Bucolo C, Puglisi G. Ocular tolerability of Eudragit RS100® and RL100® nanosuspensions as carriers for ophthalmic controlled drug delivery. Journal of Pharmaceutical Sciences. 2002; 91:2636-2641. [PubMed: 12434408]

163. Sharma UK, Verma A, Prajapati SK, Pandey H, Pandey AC. In vitro, in vivo and pharmacokinetic assessment of amikacin sulphate laden polymeric nanoparticles meant for controlled ocular drug delivery. Applied Nanoscience. 2014:1-13.

Adv Drug Deliv Rev. Author manuscript; available in PMC 2018 December 01. 
164. Müller RH, Shegokar R, Keck CM. 20 years of lipid nanoparticles (SLN \& NLC): Present state of development \& industrial applications. Current Drug Discovery Technologies. 2011; 8:207-227. [PubMed: 21291409]

165. Mandal B, Bhattacharjee H, Mittal N, Sah H, Balabathula P, Thoma LA, Wood GC. Core-shelltype lipid-polymer hybrid nanoparticles as a drug delivery platform, Nanomedicine: Nanotechnology. Biology and Medicine. 2013; 9:474-491.

166. Mehnert W, Mäder K. Solid lipid nanoparticles: Production, characterization and applications. Advanced Drug Delivery Reviews. 2001; 47:165-196. [PubMed: 11311991]

167. Müller RH, Mäder K, Gohla S. Solid lipid nanoparticles (SLN) for controlled drug delivery - A review of the state of the art. European Journal of Pharmaceutics and Biopharmaceutics. 2000; 50:161-177. [PubMed: 10840199]

168. Wissing SA, Kayser O, Müller RH. Solid lipid nanoparticles for parenteral drug delivery. Advanced Drug Delivery Reviews. 2004; 56:1257-1272. [PubMed: 15109768]

169. Wong HL, Bendayan R, Rauth AM, Li Y, Wu XY. Chemotherapy with anticancer drugs encapsulated in solid lipid nanoparticles. Advanced Drug Delivery Reviews. 2007; 59:491-504. [PubMed: 17532091]

170. Seyfoddin A, Shaw J, Al-Kassas R. Solid lipid nanoparticles for ocular drug delivery. Drug Delivery. 2010; 17:467-489. [PubMed: 20491540]

171. Attama AA, Reichl S, Müller-Goymann CC. Diclofenac sodium delivery to the eye: in vitro evaluation of novel solid lipid nanoparticle formulation using human cornea construct. International journal of pharmaceutics. 2008; 355:307-313. [PubMed: 18242022]

172. Cavalli R, Gasco MR, Chetoni P, Burgalassi S, Saettone MF. Solid lipid nanoparticles (SLN) as ocular delivery system for tobramycin. International Journal of Pharmaceutics. 2002; 238:241245. [PubMed: 11996827]

173. Gökçe EH, Sandri G, Eğrilmez S, Bonferoni MC, Güneri T, Caramella C. Cyclosporine a-loaded solid lipid nanoparticles: Ocular tolerance and in vivo drug release in rabbit eyes. Current Eye Research. 2009; 34:996-1003. [PubMed: 19958116]

174. Gokce EH, Sandri G, Bonferoni MC, Rossi S, Ferrari F, Güneri T, Caramella C. Cyclosporine A loaded SLNs: evaluation of cellular uptake and corneal cytotoxicity. International journal of pharmaceutics. 2008; 364:76-86. [PubMed: 18725276]

175. Sandri G, Bonferoni MC, Gkçe EH, Ferrari F, Rossi S, Patrini M, Caramella C. Chitosanassociated SLN: In vitro and ex vivo characterization of cyclosporine A loaded ophthalmic systems. Journal of Microencapsulation. 2010; 27:735-746. [PubMed: 21034366]

176. Başaran E, Demirel M, Sirmagül B, Yazan Y. Cyclosporine-A incorporated cationic solid lipid nanoparticles for ocular delivery. Journal of Microencapsulation. 2010; 27:37-47. [PubMed: 19545226]

177. Leonardi A, Bucolo C, Drago F, Salomone S, Pignatello R. Cationic solid lipid nanoparticles enhance ocular hypotensive effect of melatonin in rabbit. International journal of pharmaceutics. 2014

178. Kumar R, Sinha VR. Solid lipid nanoparticle: an efficient carrier for improved ocular permeation of voriconazole. Drug development and industrial pharmacy. 2016; 42:1956-1967. [PubMed: 27143048]

179. Sharma AK, Sahoo PK, Majumdar DK, Sharma N, Sharma RK, Kumar A. Fabrication and evaluation of lipid nanoparticulates for ocular delivery of a COX-2 inhibitor. Drug delivery. 2016; 23:3364-3373. [PubMed: 27128623]

180. Araújo J, Gonzalez-Mira E, Egea MA, Garcia ML, Souto EB. Optimization and physicochemical characterization of a triamcinolone acetonide-loaded NLC for ocular antiangiogenic applications. International Journal of Pharmaceutics. 2010; 393:167-175. [PubMed: 20362042]

181. Souto EB, Wissing SA, Barbosa CM, Müller RH. Evaluation of the physical stability of SLN and NLC before and after incorporation into hydrogel formulations. European Journal of Pharmaceutics and Biopharmaceutics. 2004; 58:83-90. [PubMed: 15207541]

182. Li X, Nie Sf, Kong J, Li N, Ju Cy, Pan Ws. A controlled-release ocular delivery system for ibuprofen based on nanostructured lipid carriers. International Journal of Pharmaceutics. 2008; 363:177-182. [PubMed: 18706987] 
183. Gonzalez-Mira E, Nikolić S, Calpena AC, Egea MA, Souto EB, García ML. Improved and safe transcorneal delivery of flurbiprofen by NLC and NLC-based hydrogels. Journal of Pharmaceutical Sciences. 2012; 101:707-725. [PubMed: 22012873]

184. Tártara LI, Quinteros DA, Saino V, Allemandi DA, Palma SD. Improvement of acetazolamide ocular permeation using ascorbyl laurate nanostructures as drug delivery system. Journal of Ocular Pharmacology and Therapeutics. 2012; 28:102-109. [PubMed: 22060001]

185. Shen J, Wang Y, Ping Q, Xiao Y, Huang X. Mucoadhesive effect of thiolated PEG stearate and its modified NLC for ocular drug delivery. Journal of controlled release. 2009; 137:217-223. [PubMed: 19393270]

186. Luo Q, Zhao J, Zhang X, Pan W. Nanostructured lipid carrier (NLC) coated with Chitosan Oligosaccharides and its potential use in ocular drug delivery system. International Journal of Pharmaceutics. 2011; 403:185-191. [PubMed: 20951778]

187. Araújo J, Nikolic S, Egea MA, Souto EB, Garcia ML. Nanostructured lipid carriers for triamcinolone acetonide delivery to the posterior segment of the eye. Colloids and Surfaces B: Biointerfaces. 2011; 88:150-157. [PubMed: 21764568]

188. Tian B, Luo Q, Song S, Liu D, Pan H, Zhang W, He L, Ma S, Yang X, Pan W. Novel surfacemodified nanostructured lipid carriers with partially deacetylated water-soluble chitosan for efficient ocular delivery. Journal of Pharmaceutical Sciences. 2012; 101:1040-1049. [PubMed: 22102480]

189. Shen J, Deng Y, Jin X, Ping Q, Su Z, Li L. Thiolated nanostructured lipid carriers as a potential ocular drug delivery system for cyclosporine A: Improving in vivo ocular distribution. International Journal of Pharmaceutics. 2010; 402:248-253. [PubMed: 20934499]

190. González-Mira E, Nikolić S, García ML, Egea MA, Souto EB, Calpena AC. Potential use of nanostructured lipid carriers for topical delivery of flurbiprofen. Journal of Pharmaceutical Sciences. 2011; 100:242-251. [PubMed: 20575052]

191. Liu D, Li J, Pan H, He F, Liu Z, Wu Q, Bai C, Yu S, Yang X. Potential advantages of a novel chitosan-N-acetylcysteine surface modified nanostructured lipid carrier on the performance of ophthalmic delivery of curcumin. Scientific Reports. 2016; 6

192. Leitner VM, Walker GF, Bernkop-Schnürch A. Thiolated polymers: evidence for the formation of disulphide bonds with mucus glycoproteins. European Journal of Pharmaceutics and Biopharmaceutics. 2003; 56:207-214. [PubMed: 12957634]

193. Liu R, Wang S, Sun L, Fang S, Wang J, Huang X, You Z, He X, Liu C. A novel cationic nanostructured lipid carrier for improvement of ocular bioavailability: Design, optimization, in vitro and in vivo evaluation. Journal of Drug Delivery Science and Technology. 2016; 33:28-36.

194. Ban J, Zhang Y, Huang X, Deng G, Hou D, Chen Y, Lu Z. corneal permeation properties of a charged lipid nanoparticle carrier containing dexamethasone. International Journal of Nanomedicine. 2017; 12:1329. [PubMed: 28243093]

195. Liu R, Wang S, Fang S, Wang J, Chen J, Huang X, He X, Liu C. Liquid Crystalline Nanoparticles as an Ophthalmic Delivery System for Tetrandrine: Development, Characterization, and In Vitro and In Vivo Evaluation. Nanoscale research letters. 2016; 11:254. [PubMed: 27188974]

196. Matthews OA, Shipway AN, Stoddart JF. Dendrimers - Branching out from curiosities into new technologies. Prog Polym Sci. 1998; 23:1-56.

197. Liu MJ, Frechet JMJ. Designing dendrimers for drug delivery. Pharm Sci Technol To. 1999; 2:393-401.

198. Tomalia DA, Fréchet JM. Discovery of dendrimers and dendritic polymers: A brief historical perspective*. Journal of Polymer Science Part A: Polymer Chemistry. 2002; 40:2719-2728.

199. Bai S, Thomas C, Rawat A, Ahsan F. Recent progress in dendrimer-based nanocarriers. Crit Rev Ther Drug Carrier Syst. 2006; 23:437-495. [PubMed: 17425500]

200. Soliman GM, Sharma A, Maysinger D, Kakkar A. Dendrimers and miktoarm polymers based multivalent nanocarriers for efficient and targeted drug delivery, Chemical communications (Cambridge. England). 2011; 47:9572-9587.

201. Jain NK, Gupta U. Application of dendrimer-drug complexation in the enhancement of drug solubility and bioavailability. Expert opinion on drug metabolism \& toxicology. 2008; 4:10351052. [PubMed: 18680439] 
202. Najlah M, D’Emanuele A. Synthesis of dendrimers and drug-dendrimer conjugates for drug delivery. Current opinion in drug discovery \& development. 2007; 10:756-767. [PubMed: 17987527]

203. Vandamme TF, Brobeck L. Poly (amidoamine) dendrimers as ophthalmic vehicles for ocular delivery of pilocarpine nitrate and tropicamide. Journal of controlled release. 2005; 102:23-38. [PubMed: 15653131]

204. Spataro G, Malecaze F, Turrin CO, Soler V, Duhayon C, Elena PP, Majoral JP, Caminade AM. Designing dendrimers for ocular drug delivery. European journal of medicinal chemistry. 2010; 45:326-334. [PubMed: 19889480]

205. Holden CA, Tyagi P, Thakur A, Kadam R, Jadhav G, Kompella UB, Yang H. Polyamidoamine dendrimer hydrogel for enhanced delivery of antiglaucoma drugs. Nanomedicine: nanotechnology, biology and medicine. 2012; 8:776-783.

206. Yang H, Leffler CT. Hybrid dendrimer hydrogel/poly (lactic-co-glycolic acid) nanoparticle platform: an advanced vehicle for topical delivery of antiglaucoma drugs and a likely solution to improving compliance and adherence in glaucoma management. Journal of Ocular Pharmacology and Therapeutics. 2013; 29:166-172. [PubMed: 23249385]

207. Durairaj C, Kadam RS, Chandler JW, Hutcherson SL, Kompella UB. Nanosized dendritic polyguanidilyated translocators for enhanced Solubility, Permeability, and delivery of Gatifloxacin. Investigative Ophthalmology and Visual Science. 2010; 51:5804-5816. [PubMed: 20484584]

208. Mishra V, Jain N. Acetazolamide encapsulated dendritic nano-architectures for effective glaucoma management in rabbits. International journal of pharmaceutics. 2014; 461:380-390. [PubMed: 24291772]

209. Win KY, Feng SS. Effects of particle size and surface coating on cellular uptake of polymeric nanoparticles for oral delivery of anticancer drugs. Biomaterials. 2005; 26:2713-2722. [PubMed: 15585275]

210. Gulsen D, Li CC, Chauhan A. Dispersion of DMPC liposomes in contact lenses for ophthalmic drug delivery. Current eye research. 2005; 30:1071-1080. [PubMed: 16354620]

211. Jung HJ, Chauhan A. Temperature sensitive contact lenses for triggered ophthalmic drug delivery. Biomaterials. 2012; 33:2289-2300. [PubMed: 22182750]

212. Guzman-Aranguez A, Colligris B, Pintor J. Contact lenses: promising devices for ocular drug delivery. Journal of Ocular Pharmacology and Therapeutics. 2013; 29:189-199. [PubMed: 23215541]

213. Yu S, Wang QM, Wang X, Liu D, Zhang W, Ye T, Yang X, Pan W. Liposome incorporated ion sensitive in situ gels for opthalmic delivery of timolol maleate. International journal of pharmaceutics. 2015; 480:128-136. [PubMed: 25615987]

214. Hashemi Nasra F, Khoee S, Dehghan MM, Sadeghian Chaleshtori S, Shafiee A. Preparation and Evaluation of Contact Lenses Embedded with Polycaprolactone-based Nanoparticles, for Ocular Drug Delivery. Biomacromolecules. 2016

215. Maulvi FA, Soni TG, Shah DO. Extended release of timolol from ethyl cellulose microparticles laden hydrogel contact lenses. Open Pharmaceutical Sciences Journal. 2015; 2

216. Maulvi FA, Soni TG, Shah DO. A review on therapeutic contact lenses for ocular drug delivery. Drug delivery. 2016:1-10. [PubMed: 24758139]

217. Jung HJ, Abou-Jaoude M, Carbia BE, Plummer C, Chauhan A. Glaucoma therapy by extended release of timolol from nanoparticle loaded silicone-hydrogel contact lenses. Journal of controlled release. 2013; 165:82-89. [PubMed: 23123188]

218. Hao J, Wang X, Bi Y, Teng Y, Wang J, Li F, Li Q, Zhang J, Guo F, Liu J. Fabrication of a composite system combining solid lipid nanoparticles and thermosensitive hydrogel for challenging ophthalmic drug delivery. Colloids and Surfaces B: Biointerfaces. 2014; 114:111120. [PubMed: 24176890]

219. Jain RL, Shastri J. Study of ocular drug delivery system using drug-loaded liposomes. International journal of pharmaceutical investigation. 2011; 1:35. [PubMed: 23071918]

Adv Drug Deliv Rev. Author manuscript; available in PMC 2018 December 01. 
220. Danion A, Arsenault I, Vermette P. Antibacterial activity of contact lenses bearing surfaceimmobilized layers of intact liposomes loaded with levofloxacin. Journal of pharmaceutical sciences. 2007; 96:2350-2363. [PubMed: 17541976]

221. Kapoor Y, Thomas JC, Tan G, John VT, Chauhan A. Surfactant-laden soft contact lenses for extended delivery of ophthalmic drugs. Biomaterials. 2009; 30:867-878. [PubMed: 19010533]

222. Kapoor Y, Chauhan A. Drug and surfactant transport in Cyclosporine A and Brij 98 laden pHEMA hydrogels. Journal of colloid and interface science. 2008; 322:624-633. [PubMed: 18359493]

223. Yang X, Trinh HM, Agrahari V, Sheng Y, Pal D, Mitra AK. Nanoparticle-based topical ophthalmic gel formulation for sustained release of hydrocortisone butyrate. AAPS PharmSciTech. 2016; 17:294-306. [PubMed: 26085051]

224. Bazzaz BSF, Khameneh B, Jalili-Behabadi M-m, Malaekeh-Nikouei B, Mohajeri SA. Preparation, characterization and antimicrobial study of a hydrogel (soft contact lens) material impregnated with silver nanoparticles. Contact Lens and Anterior Eye. 2014; 37:149-152. [PubMed: 24121010]

225. Zhang W, Zu D, Chen J, Peng J, Liu Y, Zhang H, Li S, Pan W. Bovine serum albumin-meloxicam nanoaggregates laden contact lenses for ophthalmic drug delivery in treatment of postcataract endophthalmitis. International journal of pharmaceutics. 2014; 475:25-34. [PubMed: 25158220]

226. Bengani LC, Chauhan A. Extended delivery of an anionic drug by contact lens loaded with a cationic surfactant. Biomaterials. 2013; 34:2814-2821. [PubMed: 23337326]

227. Lu C, Yoganathan RB, Kociolek M, Allen C. Hydrogel containing silica shell cross-linked micelles for ocular drug delivery. Journal of pharmaceutical sciences. 2013; 102:627-637. [PubMed: 23203974]

228. Behl G, Iqbal J, O’Reilly NJ, McLoughlin P, Fitzhenry L. Synthesis and characterization of poly (2-hydroxyethylmethacrylate) contact lenses containing chitosan nanoparticles as an ocular delivery system for dexamethasone sodium phosphate. Pharmaceutical research. 2016; 33:16381648. [PubMed: 26964548]

229. Danion A, Brochu H, Martin Y, Vermette P. Fabrication and characterization of contact lenses bearing surface- immobilized layers of intact liposomes. Journal of Biomedical Materials Research Part A. 2007; 82:41-51. [PubMed: 17265438]

230. Danion A, Doillon CJ, Giasson CJ, Djouahra S, Sauvageau P, Paradis R, Vermette P. Biocompatibility and light transmission of liposomal lenses. Optometry \& Vision Science. 2007; 84:954-961. [PubMed: 18049361]

231. Garhwal R, Shady SF, Ellis EJ, Ellis JY, Leahy CD, McCarthy SP, Crawford KS, Gaines P. Sustained ocular delivery of ciprofloxacin using nanospheres and conventional contact lens materials. Investigative ophthalmology \& visual science. 2012; 53:1341-1352. [PubMed: 22266514]

232. Yavuz B, Bozdağ Pehlivan S, Kaffashi A, Çalamak S, Ulubayram K, Palaska E, Çakmak HB, Ünlü N. In vivo tissue distribution and efficacy studies for cyclosporin A loaded nano-decorated subconjunctival implants. Drug delivery. 2016; 23:3279-3284. [PubMed: 27027148]

233. U. FDAGuidance for Industry: Considering Whether an FDA-Regulated Product Involves the Application of NanotechnologyJun, 2014

234. Kunzmann A, Andersson B, Thurnherr T, Krug H, Scheynius A, Fadeel B. Toxicology of engineered nanomaterials: focus on biocompatibility, biodistribution and biodegradation. Biochimica et Biophysica Acta (BBA)-General Subjects. 2011; 1810:361-373. [PubMed: 20435096]

235. U. FDANanotechnology Task Force Report 20072007

236. C.o.C. Academics. Report of the Expert Panel on Nanotechnology. Small is Different: A Science Perspective on the Regulatory Challenges of the Nanoscale. 2008

237. SadriehN. FDA Considerations For Regulation Of Nanomaterial Containing ProductsCDER, FDA; 2006

238. Prow TW. Toxicity of nanomaterials to the eye. Wiley Interdisciplinary Reviews: Nanomedicine and Nanobiotechnology. 2010; 2:317-333. [PubMed: 20077524] 
239. Bertrand N, Wu J, Xu X, Kamaly N, Farokhzad OC. Cancer nanotechnology: the impact of passive and active targeting in the era of modern cancer biology. Advanced drug delivery reviews. 2014; 66:2-25. [PubMed: 24270007]

240. Davis ME, Shin DM. Nanoparticle therapeutics: an emerging treatment modality for cancer. Nature reviews Drug discovery. 2008; 7:771-782. [PubMed: 18758474]

241. Bertrand N, Leroux JC. The journey of a drug-carrier in the body: an anatomo-physiological perspective. Journal of Controlled Release. 2012; 161:152-163. [PubMed: 22001607]

242. Gu F, Zhang L, Teply BA, Mann N, Wang A, Radovic-Moreno AF, Langer R, Farokhzad OC. Precise engineering of targeted nanoparticles by using self-assembled biointegrated block copolymers. Proceedings of the National Academy of Sciences. 2008; 105:2586-2591.

243. Jiang W, Kim BY, Rutka JT, Chan WC. Nanoparticle-mediated cellular response is sizedependent. Nature nanotechnology. 2008; 3:145-150.

244. Valencia PM, Hanewich-Hollatz MH, Gao W, Karim F, Langer R, Karnik R, Farokhzad OC. Effects of ligands with different water solubilities on self-assembly and properties of targeted nanoparticles. Biomaterials. 2011; 32:6226-6233. [PubMed: 21658757]

245. Wu L, Zhang J, Watanabe W. Physical and chemical stability of drug nanoparticles. Advanced drug delivery reviews. 2011; 63:456-469. [PubMed: 21315781]

246. Losa C, Calvo P, Castro E, VILA-JATO J, Alonso M. Improvement of ocular penetration of amikacin sulphate by association to poly (butylcyanoacrylate) nanoparticles. Journal of pharmacy and pharmacology. 1991; 43:548-552. [PubMed: 1681069]

247. Calvo P, Thomas C, Alonso MJ, Vila-Jato J, Robinson JR. Study of the mechanism of interaction of poly (-caprolactone) nanocapsules with the cornea by confocal laser scanning microscopy. International journal of pharmaceutics. 1994; 103:283-291.

248. Calvo P, Vila-Jato JL, Alonso MaJ. Evaluation of cationic polymer-coated nanocapsules as ocular drug carriers. International Journal of Pharmaceutics. 1997; 153:41-50.

249. Pignatello R, Bucolo C, Ferrara P, Maltese A, Puleo A, Puglisi G. Eudragit RS100® nanosuspensions for the ophthalmic controlled delivery of ibuprofen. European Journal of Pharmaceutical Sciences. 2002; 16:53-61. [PubMed: 12113891]

250. Cavalli R, Gasco MR, Chetoni P, Burgalassi S, Saettone MF. Solid lipid nanoparticles (SLN) as ocular delivery system for tobramycin. International journal of pharmaceutics. 2002; 238:241245. [PubMed: 11996827]

251. Raviña M, Cubillo E, Olmeda D, Novoa-Carballal R, Fernandez-Megia E, Riguera R, Sánchez A, Cano A, Alonso MJ. Hyaluronic acid/chitosan-g-poly (ethylene glycol) nanoparticles for gene therapy: an application for pDNA and siRNA delivery. Pharmaceutical research. 2010; 27:25442555. [PubMed: 20857179]

252. Diebold Y, Jarrín M, Sáez V, Carvalho EL, Orea M, Calonge M, Seijo B, Alonso MJ. Ocular drug delivery by liposome-chitosan nanoparticle complexes (LCS-NP). Biomaterials. 2007; 28:15531564. [PubMed: 17169422]

253. Yuan XB, Yuan YB, Jiang W, Liu J, Tian EJ, Shun HM, Huang DH, Yuan XY, Li H, Sheng J. Preparation of rapamycin-loaded chitosan/PLA nanoparticles for immunosuppression in corneal transplantation. International journal of pharmaceutics. 2008; 349:241-248. [PubMed: 17919859]

254. Li N, Zhuang C, Wang M, Sun X, Nie S, Pan W. Liposome coated with low molecular weight chitosan and its potential use in ocular drug delivery. International journal of pharmaceutics. 2009; 379:131-138. [PubMed: 19559775]

255. Leonardi A, Bucolo C, Drago F, Salomone S, Pignatello R. Cationic solid lipid nanoparticles enhance ocular hypotensive effect of melatonin in rabbit. International journal of pharmaceutics. 2015; 478:180-186. [PubMed: 25448580]

256. De Campos AM, Diebold Y, Carvalho EL, Sánchez A, José Alonso M. Chitosan nanoparticles as new ocular drug delivery systems: in vitro stability, in vivo fate, and cellular toxicity. Pharmaceutical research. 2004; 21:803-810. [PubMed: 15180338]

257. Mitra RN, Han Z, Merwin M, Al Taai M, Conley SM, Naash MI. Synthesis and characterization of glycol chitosan DNA nanoparticles for retinal gene delivery. ChemMedChem. 2014; 9:189196. [PubMed: 24203490]

Adv Drug Deliv Rev. Author manuscript; available in PMC 2018 December 01. 
258. Dilbaghi N, Kaur H, Ahuja M, Kumar S. Evaluation of tropicamide-loaded tamarind seed xyloglucan nanoaggregates for ophthalmic delivery. Carbohydrate polymers. 2013; 94:286-291. [PubMed: 23544540]

259. Shinde U, Ahmed MH, Singh K. Development of dorzolamide loaded 6-O-carboxymethyl chitosan nanoparticles for open angle glaucoma. Journal of drug delivery. 2013; 2013

260. Ibrahim MM, Abd-Elgawad AEH, Soliman OA, Jablonski MM. Novel topical ophthalmic formulations for management of glaucoma. Pharmaceutical research. 2013; 30:2818-2831. [PubMed: 23771565]

261. Sharma UK, Verma A, Prajapati SK, Pandey H, Pandey AC. In vitro, in vivo and pharmacokinetic assessment of amikacin sulphate laden polymeric nanoparticles meant for controlled ocular drug delivery. Applied Nanoscience. 2015; 5:143-155.

262. Jain K, Suresh Kumar R, Sood S, Dhyanandhan G. Betaxolol hydrochloride loaded chitosan nanoparticles for ocular delivery and their anti-glaucoma efficacy. Current drug delivery. 2013; 10:493-499. [PubMed: 23410069]

263. Vega E, Gamisans F, Garcia M, Chauvet A, Lacoulonche F, Egea M. PLGA nanospheres for the ocular delivery of flurbiprofen: drug release and interactions. Journal of Pharmaceutical Sciences. 2008; 97:5306-5317. [PubMed: 18425815]

264. Cheng Y, Qu H, Ma M, Xu Z, Xu P, Fang Y, Xu T. Polyamidoamine (PAMAM) dendrimers as biocompatible carriers of quinolone antimicrobials: an in vitro study. European journal of medicinal chemistry. 2007; 42:1032-1038. [PubMed: 17336426]

265. Fayek HH. Formulation and evaluation of betamethasone sodium phosphate loaded nanoparticles for ophthalmic delivery. 2011

266. Taylor MJ, Tanna S, Sahota T. In vivo study of a polymeric glucose- sensitive insulin delivery system using a rat model. Journal of pharmaceutical sciences. 2010; 99:4215-4227. [PubMed: 20737629]

267. Katiyar S, Pandit J, Mondal RS, Mishra AK, Chuttani K, Aqil M, Ali A, Sultana Y. In situ gelling dorzolamide loaded chitosan nanoparticles for the treatment of glaucoma. Carbohydrate polymers. 2014; 102:117-124. [PubMed: 24507263]

268. Moya-Ortega MD, Alves TF, Alvarez-Lorenzo C, Concheiro A, Stefánsson E, Thorsteinsdóttir M, Loftsson T. Dexamethasone eye drops containing $\gamma$-cyclodextrin-based nanogels. International journal of pharmaceutics. 2013; 441:507-515. [PubMed: 23149258]

269. Kim J, Conway A, Chauhan A. Extended delivery of ophthalmic drugs by silicone hydrogel contact lenses. Biomaterials. 2008; 29:2259-2269. [PubMed: 18289662]

270. Nagai N, Ito Y, Okamoto N, Shimomura Y. A nanoparticle formulation reduces the corneal toxicity of indomethacin eye drops and enhances its corneal permeability. Toxicology. 2014; 319:53-62. [PubMed: 24598350]

271. Kalam MA. Development of chitosan nanoparticles coated with hyaluronic acid for topical ocular delivery of dexamethasone. International journal of biological macromolecules. 2016; 89:127136. [PubMed: 27126165]

272. De Salamanca AE, Diebold Y, Calonge M, García-Vazquez C, Callejo S, Vila A, Alonso MJ. Chitosan nanoparticles as a potential drug delivery system for the ocular surface: toxicity, uptake mechanism and in vivo tolerance. Investigative ophthalmology \& visual science. 2006; 47:14161425. [PubMed: 16565375]

273. Kalam MA. The potential application of hyaluronic acid coated chitosan nanoparticles in ocular delivery of dexamethasone. International journal of biological macromolecules. 2016; 89:559568. [PubMed: 27164496]

274. Yenice İ, Mocan MC, Palaska E, Bochot A, Bilensoy E, Vural İ, İrkeç M, Hıncal AA. Hyaluronic acid coated poly--caprolactone nanospheres deliver high concentrations of cyclosporine A into the cornea. Experimental eye research. 2008; 87:162-167. [PubMed: 18675411]

275. Harmia T, Kreuter J, Speiser P, Boye T, Gurny R, Kubi A. Enhancement of the myotic response of rabbits with pilocarpine-loaded polybutylcyanoacrylate nanoparticles. International journal of pharmaceutics. 1986; 33:187-193.

Adv Drug Deliv Rev. Author manuscript; available in PMC 2018 December 01. 
276. Marchal-Heussler L, Maincent P, Hoffman M, Spittler J, Couvreur P. Antiglaucomatous activity of betaxolol chlorhydrate sorbed onto different isobutylcyanoacrylate nanoparticle preparations. International journal of pharmaceutics. 1990; 58:115-122.

277. Rajendran N, Natrajan R, Kumar R, Selvaraj S. Acyclovir-loaded chitosan nanoparticles for ocular delivery. Asian Journal of Pharmaceutics. 2010; 4:220.

278. Zhu X, Su M, Tang S, Wang L, Liang X, Meng F, Hong Y, Xu Z. Synthesis of thiolated chitosan and preparation nanoparticles with sodium alginate for ocular drug delivery. 2012

279. Wadhwa S, Paliwal R, Paliwal SR, Vyas S. Hyaluronic acid modified chitosan nanoparticles for effective management of glaucoma: development, characterization, and evaluation. Journal of drug targeting. 2010; 18:292-302. [PubMed: 19943753]

280. Zhang P, Liu X, Hu W, Bai Y, Zhang L. Preparation and evaluation of naringenin-loaded sulfobutylether- $\beta$-cyclodextrin/chitosan nanoparticles for ocular drug delivery. Carbohydrate polymers. 2016; 149:224-230. [PubMed: 27261746]

281. Upadhayay P, Kumar M, Pathak K. Norfloxacin Loaded pH Triggered Nanoparticulate in-situ Gel for Extraocular Bacterial Infections: Optimization, Ocular Irritancy and Corneal Toxicity. Iranian Journal of Pharmaceutical Research: IJPR. 2016; 15:3.

282. Gulsen D, Chauhan A. Ophthalmic drug delivery through contact lenses. Investigative ophthalmology \& visual science. 2004; 45:2342-2347. [PubMed: 15223815]

283. Nasr FH, Khoee S, Dehghan MM, Chaleshtori SS, Shafiee A. Preparation and evaluation of contact lenses embedded with polycaprolactone-based nanoparticles for ocular drug delivery. Biomacromolecules. 2016; 17:485-495. [PubMed: 26652301] 


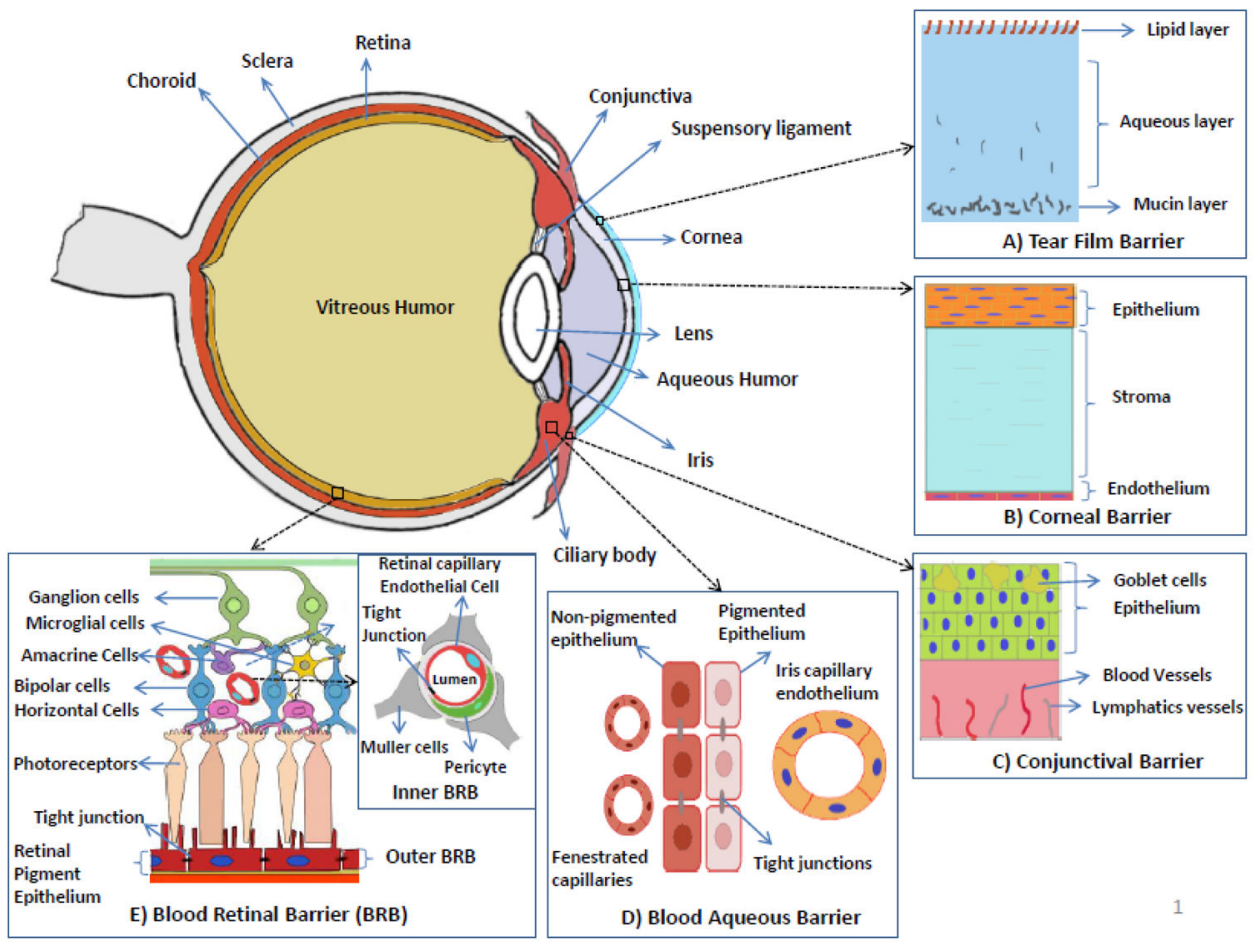

Fig. 1.

Structure of the anterior segment of the eye and ocular barriers for drug delivery. A) Tear film barrier: Main components of the tear film include mucins, water and lipid, and acts a defensive barrier to the foreign-object access to the cornea and conjunctiva. B) Corneal barrier: avascular and comprised of three major layers which are epithelium (multiple layers stacked on each other), stroma and endothelium (single layer). Acts as a barrier preventing the drug absorption from the lacrimal fluid into the anterior chamber after the topical administration. C) Conjunctival barrier: mucous membrane consisting of conjunctival epithelium (2-3 layers thick), and an underlying vascularized connective tissue. Acts a barrier to the topically administered drugs and relatively in-efficient compared to the corneal barrier. D) Blood-aqueous barrier: located in the anterior segment of the eye. Formed by the capillary endothelium in the iris, and the ciliary epithelium which both contain tight junctions. The barrier is relatively inefficient compared to the blood retinal barrier and small molecules can reach the aqueous humor by permeation through fenestrated capillaries in the ciliary processes. E) Blood-retinal barrier (BRB): located in the poster segment of the eye. Formed by the retinal pigment epithelium (outer BRB) and the endothelial membrane of the retinal blood vessels (inner BRB), both contain tight junctions. The tight junctions restrict the entry of the drugs from the blood (systemic) into the retina/aqueous humor. 


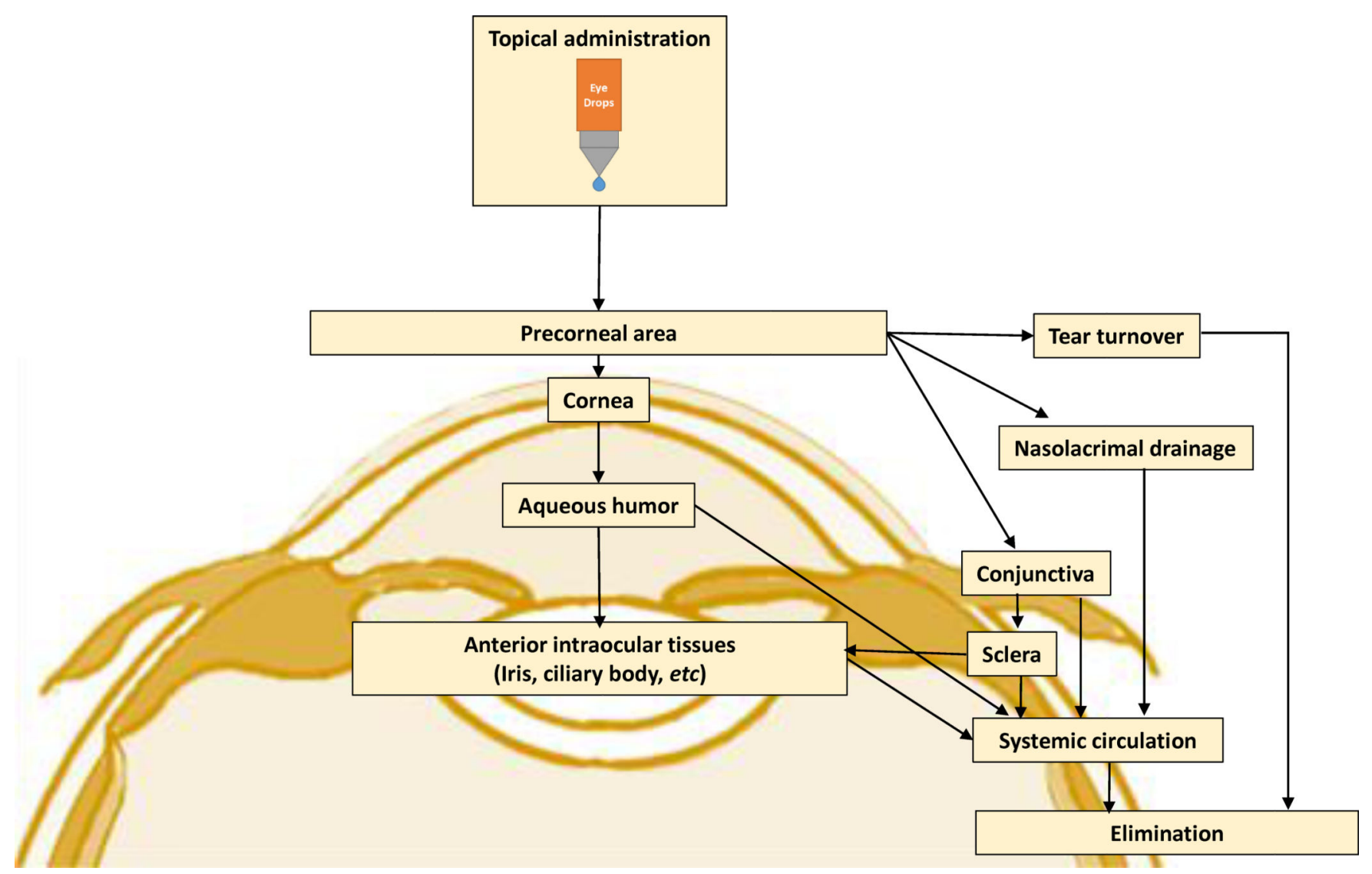

Fig. 2.

Flow chart presenting the drug pathway into the eye after topical application. 

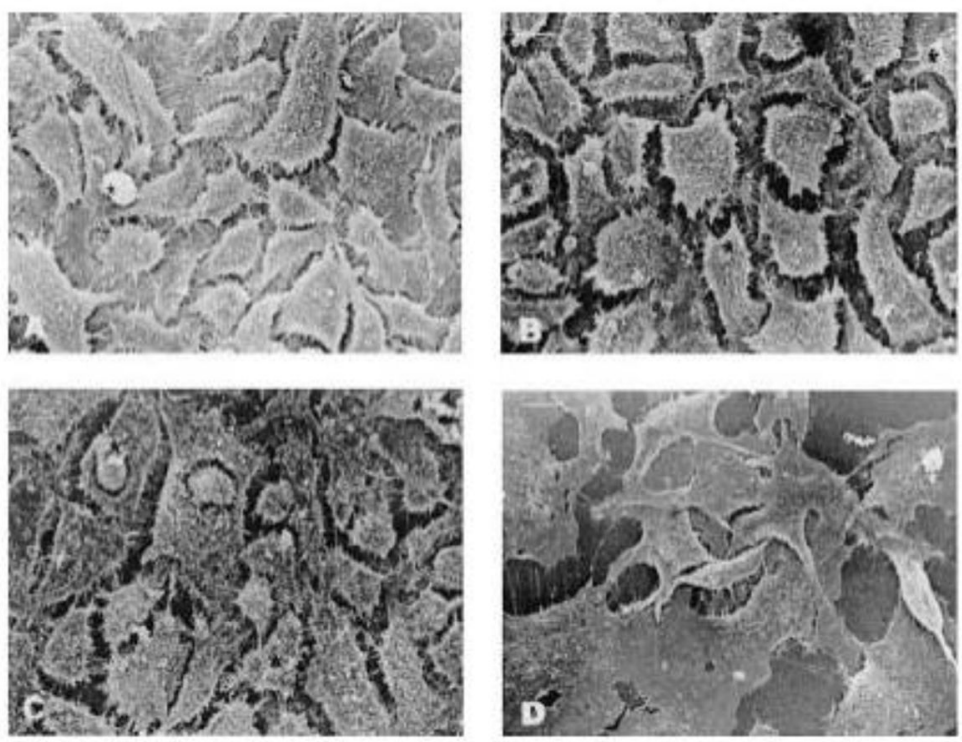

Fig. 3.

Scanning electron microphotographs of Chang cells exposed to culture medium, chitosan nanoparticles, and benzalkonium chloride for $24 \mathrm{~h}$. (A) (Negative control, culture medium) Cells showing abundant microvilli and intact membrane details. (B) (Chitosan nanoparticles, $0.25 \mathrm{mg} / \mathrm{ml}$ ) cells showing well-preserved morphology, an intact cell surface, and abundant microvilli, as expected for an epithelial cell. (C) (Chitosan nanoparticles, $1 \mathrm{mg} / \mathrm{ml}$ ). (D) (Positive control, benzalkonium chloride) Cells were flat and showed absence of microvilli and broken membrane. Magnification $\times 750$ (bar $=15 \mu \mathrm{m})$. [Reprinted from "Pharmaceutical research, Chitosan nanoparticles as new ocular drug delivery systems: in vitro stability, in vivo fate, and cellular toxicity, 21.5 (2004): 803-810. De Campos, A. M., Diebold, Y., Carvalho, E. L., Sánchez, A., \& José Alonso, M. c 2004 Plenum Publishing Corporation" With permission of Springer.] 

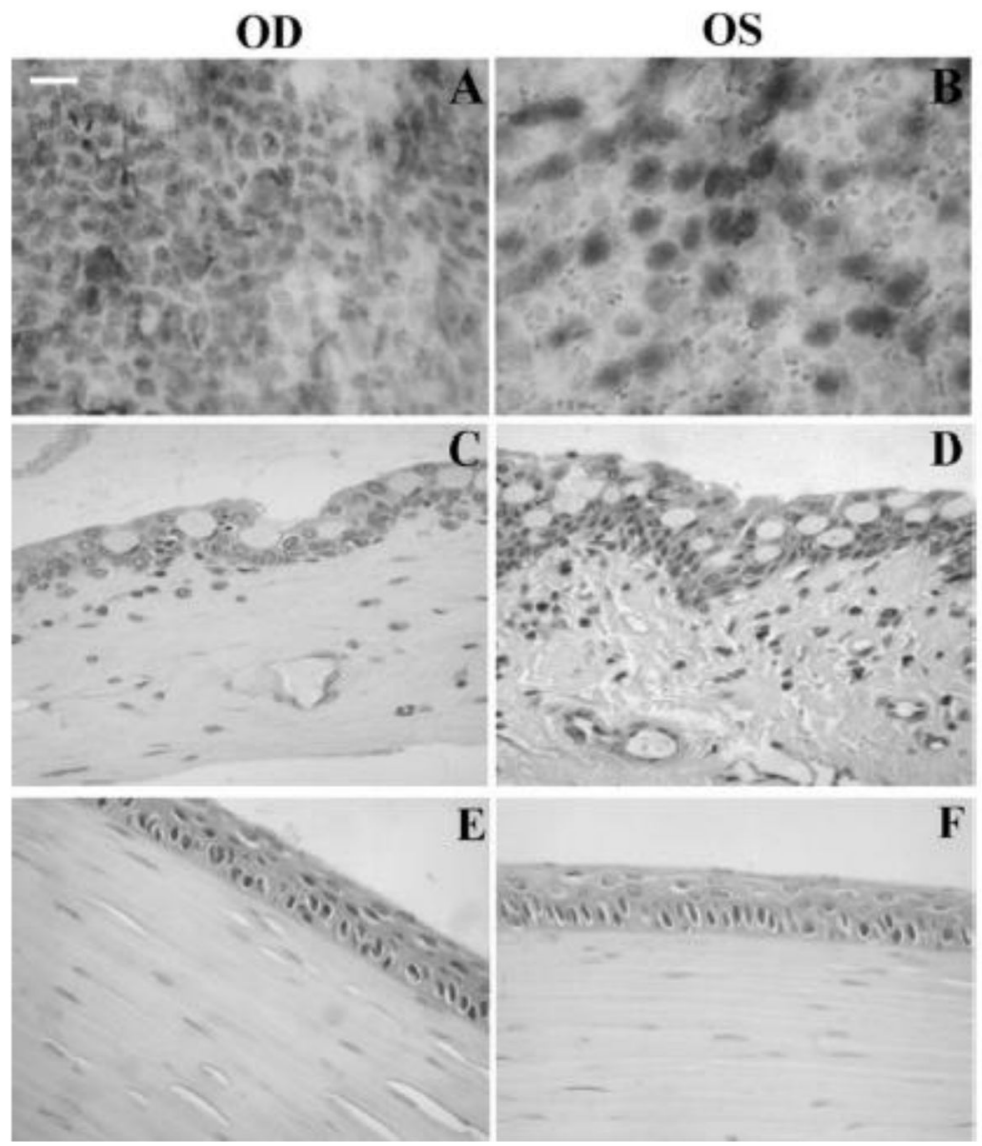

Fig. 4.

Ocular surface structures of CSNP-treated (OD) and control (OS) rabbit eyes. Rabbits were exposed to CSNPs for 24 hours. Representative conjunctival impression cytology (A, B) and conjunctival (C, D) and corneal (E, F) sections are shown. Conjunctival and corneal epithelia from both control and treated eyes displayed normal cell layers and morphology. No signs of tissue edema were observed in any structure studied after exposure to CSNPs compared with controls. Scale bar, $100 \mu \mathrm{m}$. [Reprinted from De Salamanca, Amalia Enríquez, et al.

"Chitosan nanoparticles as a potential drug delivery system for the ocular surface: toxicity, uptake mechanism and in vivo tolerance." Investigative ophthalmology \& visual science 47.4 (2006): 1416-1425. Copyright (C Association for Research in Vision and Ophthalmology] 
C

$$
\begin{aligned}
& \text { No visible } \\
& \text { fluorescence }
\end{aligned}
$$

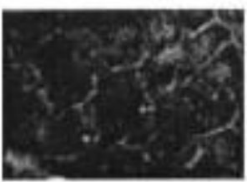

No visible fluoreseence

\section{B}

No visible

fluorescence
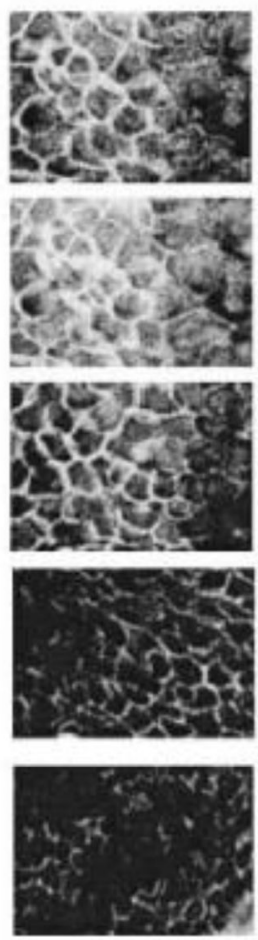

A
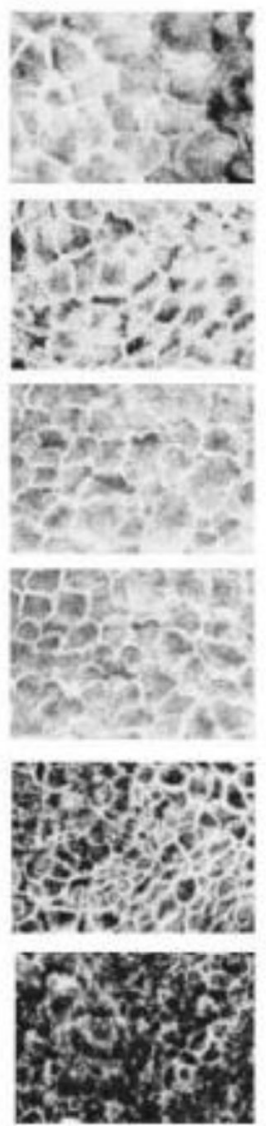

Fig. 5.

Confocal fluorescence images at different levels from the rabbit corneal epithelium (5 $\mu \mathrm{m}$ sequential cross sections from the corneal surface) at $1 \mathrm{~h}$ postinstillation of (C) Chitosanfluorescein nanoparticles, (B) Chitosan-fluorescein solution. (A) image of cross section of a nontreated cornea. Round fluorescent spots corresponding to the chitosan-fluorescein nanoparticles were observed inside the cells. [Reprinted from "Pharmaceutical research, Chitosan nanoparticles as new ocular drug delivery systems: in vitro stability, in vivo fate, and cellular toxicity, 21.5 (2004): 803-810. De Campos, A. M., Diebold, Y., Carvalho, E. L., Sánchez, A., \& José Alonso, M. c 2004 Plenum Publishing Corporation" With permission of Springer.] 


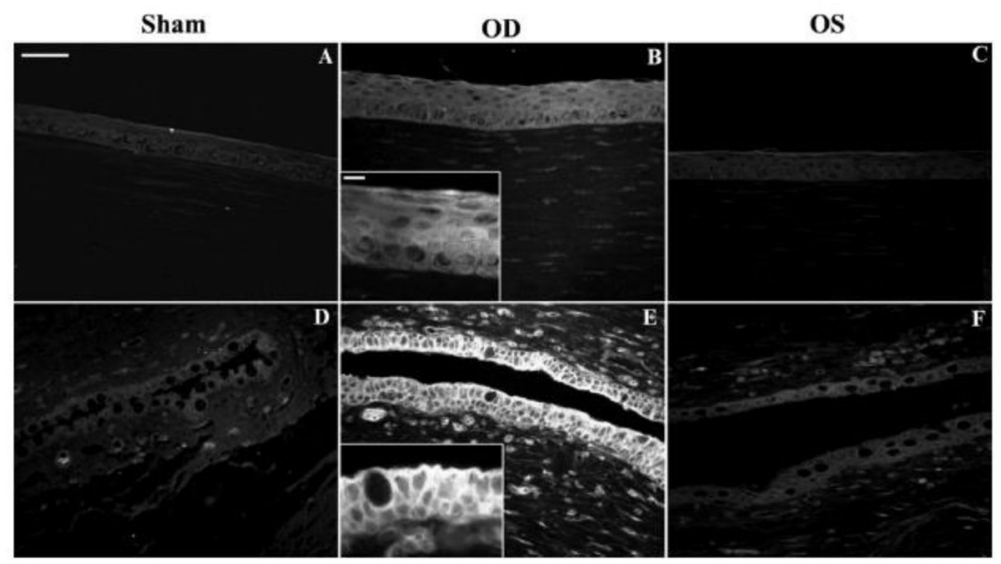

Fig. 6.

Chitosan nanoparticle in vivo uptake. Fluorescence microscopy of ocular surface structures of sham-treated (A, D), CSNP-treated (B, E), and contralateral control (C, F) rabbit eyes. Representative corneal (A-C) and conjunctival (D-F) sections are shown. No fluorescence was detected in sham control corneas (A) or conjunctivas (D). (B) Corneal epithelial cells of CSNP-treated rabbits were uniformly fluorescent. (B, inset): enlargement showing a detail of corneal epithelial fluorescence pattern. (E) Fluorescence in conjunctival epithelial cells was intense in apical cell membranes and positive along the basolateral cell membrane. (E, inset) Enlargement showing the basolateral membrane fluorescence staining in goblet and non-goblet cells. (C, F) Some fluorescence was detected in corneal and conjunctival epithelial cells from contralateral control eye (OS), although much less intense than in the treated (OD) eye. Scale bar (A-F) $50 \mu \mathrm{m}$; insets: $10 \mu \mathrm{m}$ ). The in vivo uptake by conjunctival and corneal epithelia was confirmed from these fluorescence microscopy images of eyeball and lids sections confirmed. [Reprinted from De Salamanca, Amalia Enrique, et al.

"Chitosan nanoparticles as a potential drug delivery system for the ocular surface: toxicity, uptake mechanism and in vivo tolerance." Investigative ophthalmology \& visual science 47.4 (2006): 1416-1425. Copyright (C) Association for Research in Vision and Ophthalmology.] 
$37^{\circ} \mathrm{C}$

A)

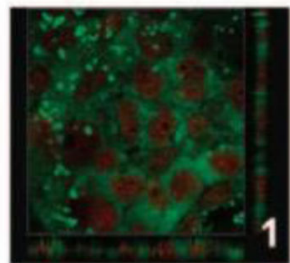

B)

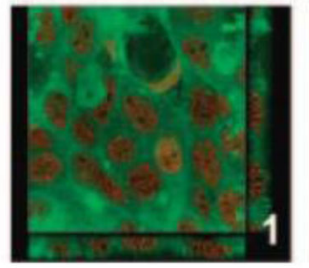

$4^{\circ} \mathrm{C}$
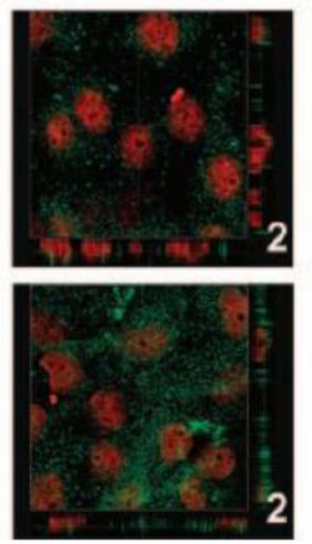

$4^{\circ} \mathrm{C}+\mathrm{Ab}$
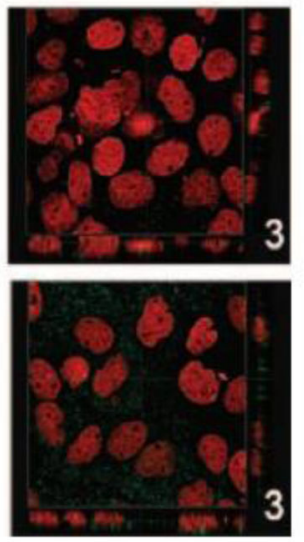

Fig. 7.

Confocal images showing the internalization of (A) Hyaluronic acid: Chitosan oligomer (mass ratio, 1:2), and (B) Hyaluronic acid: Chitosan (mass ratio, 2:1) NP in HCE cells. The NPs were incubated at $37^{\circ} \mathrm{C}(1), 4^{\circ} \mathrm{C}(2)$, and $4^{\circ} \mathrm{C}$ after blocking of the $\mathrm{CD} 44$ receptor with the monoclonal antibody Hermes-1 (3). The images show cross sections in the $\mathrm{x}-\mathrm{y}$ and $\mathrm{x}-\mathrm{Z}$ axes of the series. HA was labeled with fluoresceinamine (green), and the cell nuclei were stained with propidium iodide (red). Magnification, 63. An extensive internalization of nanoparticles was observed at $37^{\circ} \mathrm{C}$ evidencing endocytic uptake of the nanoparticles into the cells (B1). The observed minor cell association at $4^{\circ} \mathrm{C}$ could be attributed to the receptormediated uptake of the particles (B2). However, when the study was repeated at $4^{\circ} \mathrm{C}$ in presence of CD44 receptor blocker (monoclonal antibody Hermes-1), a negligible uptake of nanoparticles was observed (B3), thus concluding the internationalization mechanism as CD44 receptor-mediated endocytosis. [Reprinted from de la Fuente, Maria, Begona Seijo, and Maria J. Alonso. "Novel hyaluronic acid-chitosan nanoparticles for ocular gene therapy." Investigative ophthalmology \& visual science 49.5 (2008): 2016-2024. Copyright (C) Association for Research in Vision and Ophthalmology] 
A

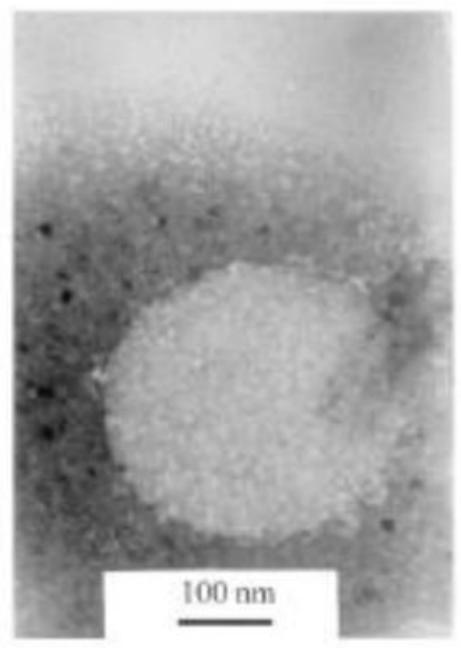

C

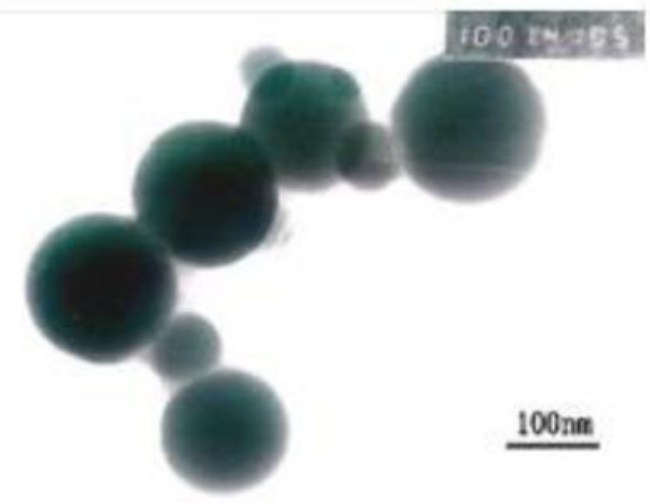

B

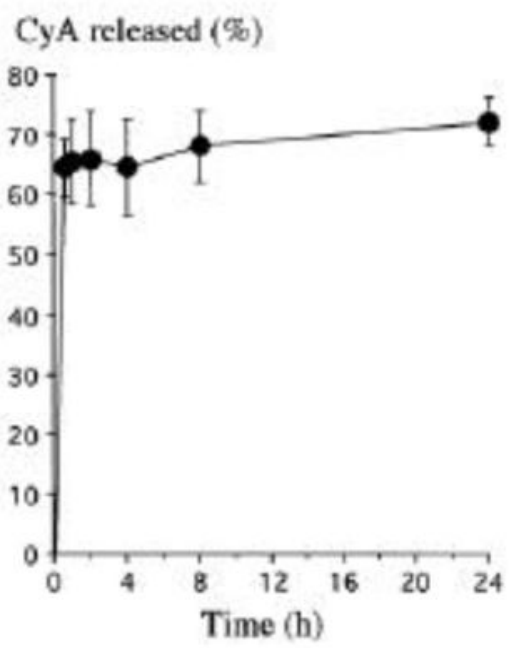

D

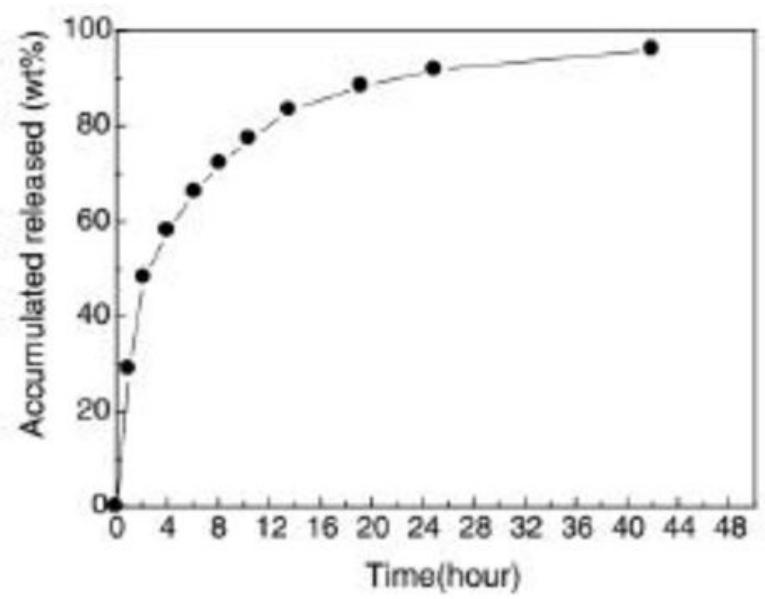

Fig. 8.

Transmission electron micrograph of the CsA-loaded CS nanoparticles (prepared by ionotropic gelation method) (A). In vitro CsA release profile from CyA-loaded CS nanoparticles (B) [Reprinted from International journal of pharmaceutics, 224(1), (2001). De Campos, A. M., Sánchez, A., \& Alonso, M. J. Chitosan nanoparticles: a new vehicle for the improvement of the delivery of drugs to the ocular surface. Application to cyclosporine A. 159-168. Copyright (C) 2001 Elsevier Science B.V., with permission from Elsevier]. TEM micrographs of cholesterol modified chitosan nanoparticles (formed by self-aggregation) (C). In vitro $\mathrm{CsA}$ release from $\mathrm{CS}-\mathrm{CH}$ self-aggregated nanoparticles (D) [Reprinted from Carbohydrate Polymers 65.3 (2006). Yuan, X. B., Li, H., \& Yuan, Y. B. Preparation of cholesterol-modified chitosan self-aggregated nanoparticles for delivery of drugs to ocular surface. 337-345 Copyright (C) 2006 Elsevier Ltd, with permission from Elsevier.] 

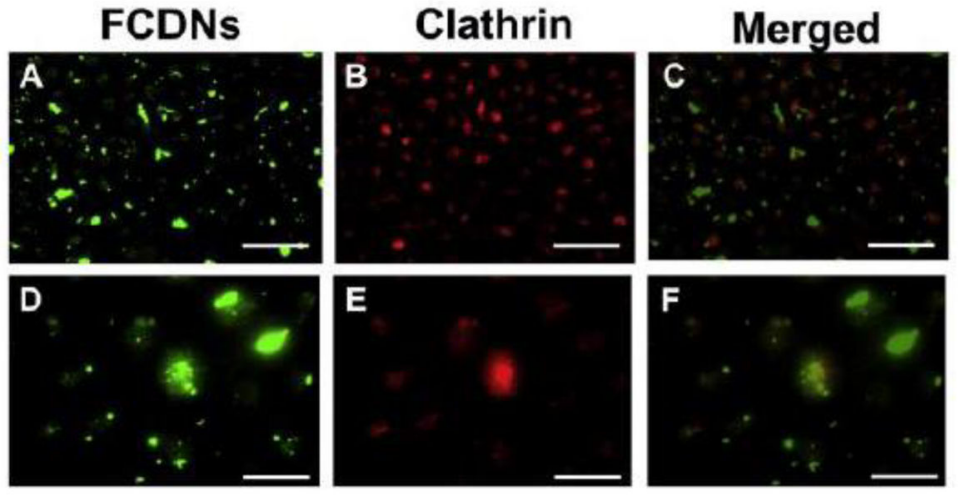

Fig. 9.

Uptake of FITC labeled Chitosan-Dextran sulfate Nanoparticles (FCDNs) occurs by a clathrin-dependent mechanism. (A, D) Images taken incubation with of cells FCDNs for 30 min at $37 \mathrm{C}$. (B, E) Cells are stained with clathrin (red). (C, F) Merged image showing the colocalization of FCDNs and clathrin (i.e., yellow spots). Magnification: $(A-C) \times 20$; (D-F) $\times 63$. Scale bar: $(A-C)=50 \mu \mathrm{m} ;(D-F)=20 \mu \mathrm{m}$. Images shown are typical results after 3 independent trials. [Reprinted from Colloids and Surfaces B: Biointerfaces 149 (2017). Chaiyasan, W., Praputbut, S., Kompella, U. B., Srinivas, S. P., \& Tiyaboonchai, W. Penetration of mucoadhesive chitosan-dextran sulfate nanoparticles into the porcine cornea. 288-296. (C) 2016 Elsevier B.V. with permission from Elsevier.] 


\section{Phase contrast}

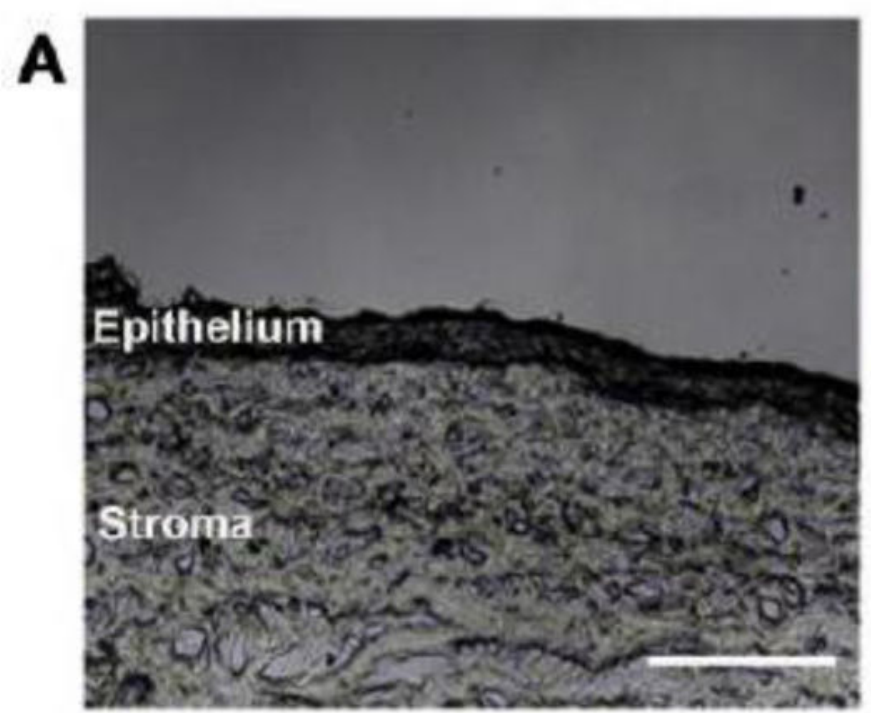

B

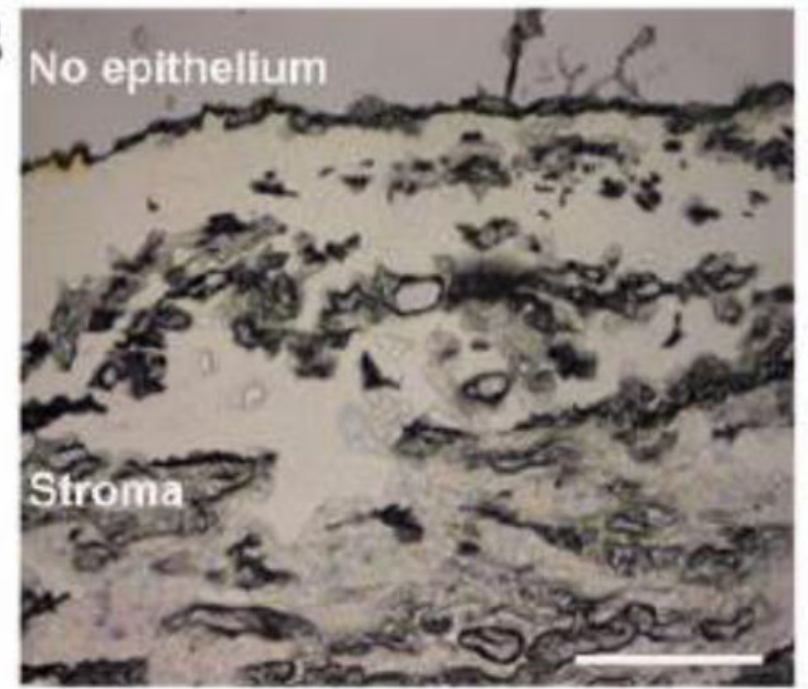

Fluorescence
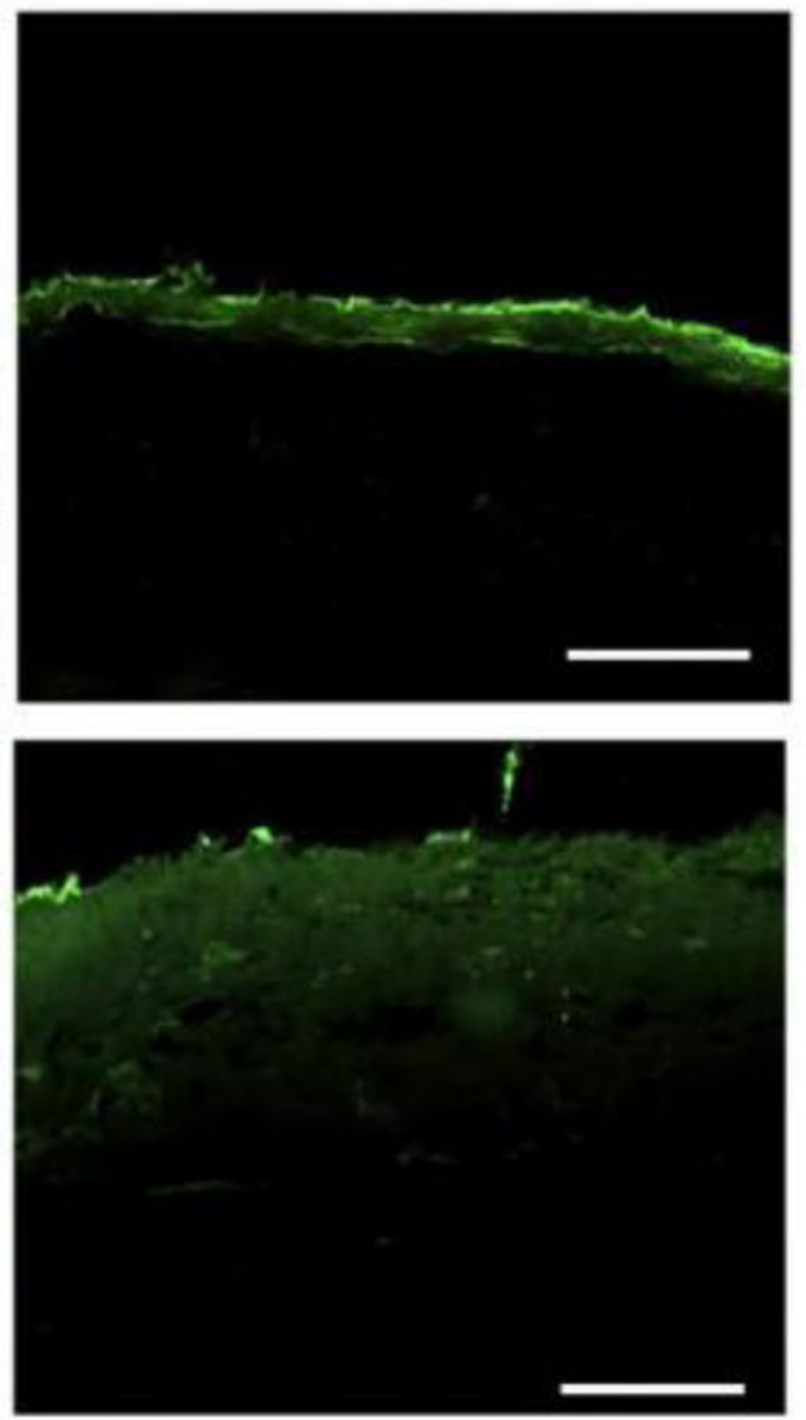

Fig. 10.

Cross section of the porcine cornea after its exposure to FCDNs for 3 h. (A) Intact corneal epithelium. (B) After removal of the corneal epithelium. Scale bar $=250 \mu \mathrm{m}$. Images shown are typical results after 3 independent trials. [Reprinted from Colloids and Surfaces B: Biointerfaces 149 (2017). Chaiyasan, W., Praputbut, S., Kompella, U. B., Srinivas, S. P., \& Tiyaboonchai, W. Penetration of mucoadhesive chitosan-dextran sulfate nanoparticles into the porcine cornea. 288-296. (C) 2016 Elsevier B.V. with permission from Elsevier.] 

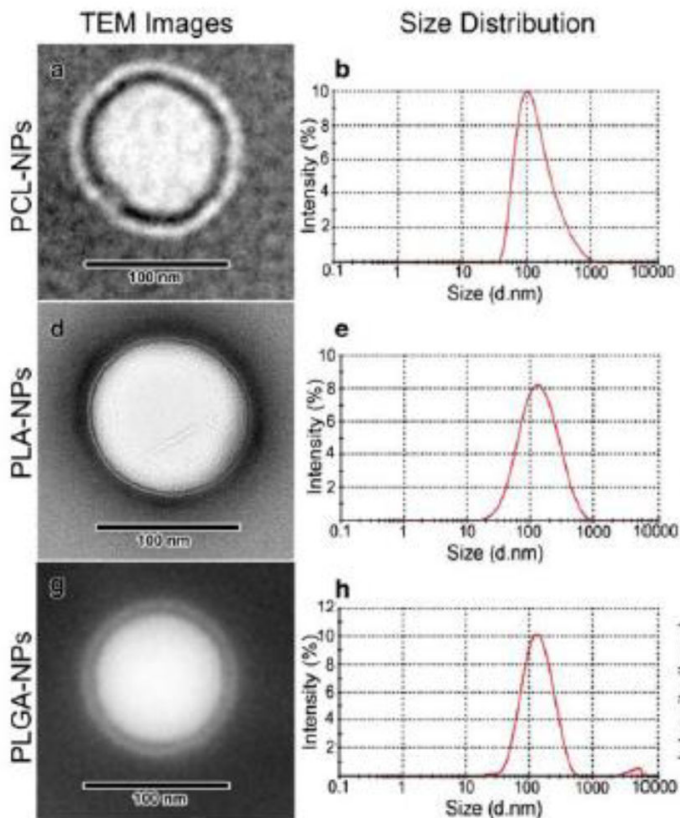

\section{Zeta Potential Distribution}

c
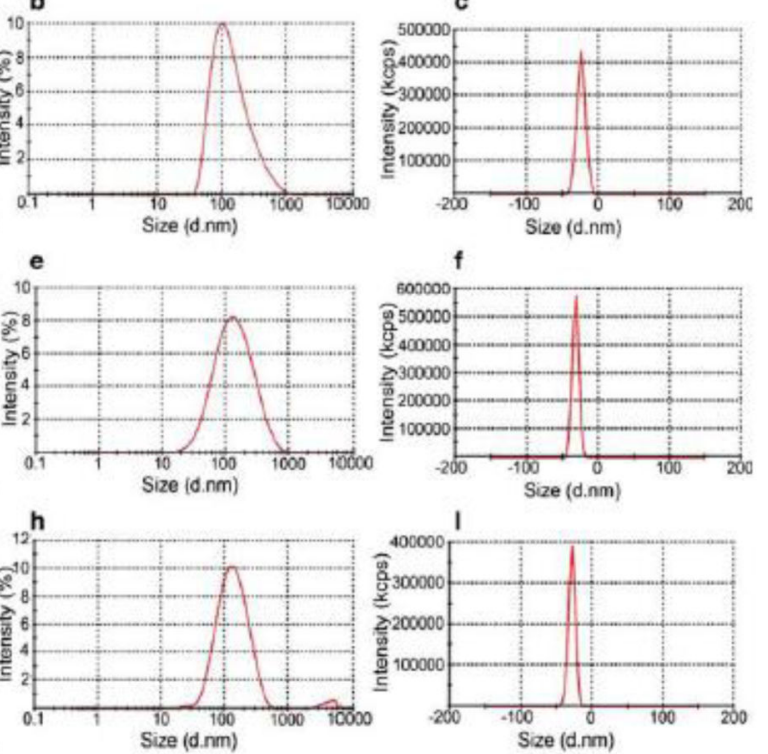

Fig. 11.

TEM images, size and zeta potential distribution curves for brimonidine-loaded nanoparticles. TEM image (a), size distribution curve (b) and zeta potential distribution curve (c) of PCL-nanoparticles, TEM image (d), size distribution curve (e) and zeta potential distribution curve (f) of PLA-nanoparticles, TEM image (g), size distribution curve (h) and zeta potential distribution curve (i) of PLGA-nanoparticles. [Reprinted permission "Pharmaceutical research. Novel topical ophthalmic formulations for management of glaucoma. 30.11 (2013): 2818-2831. Ibrahim, M. M., Abd-Elgawad, A. E. H., Soliman, O. A., \& Jablonski, M. M. () Springer Science+Business Media New York 2013.” With permission of Springer] 

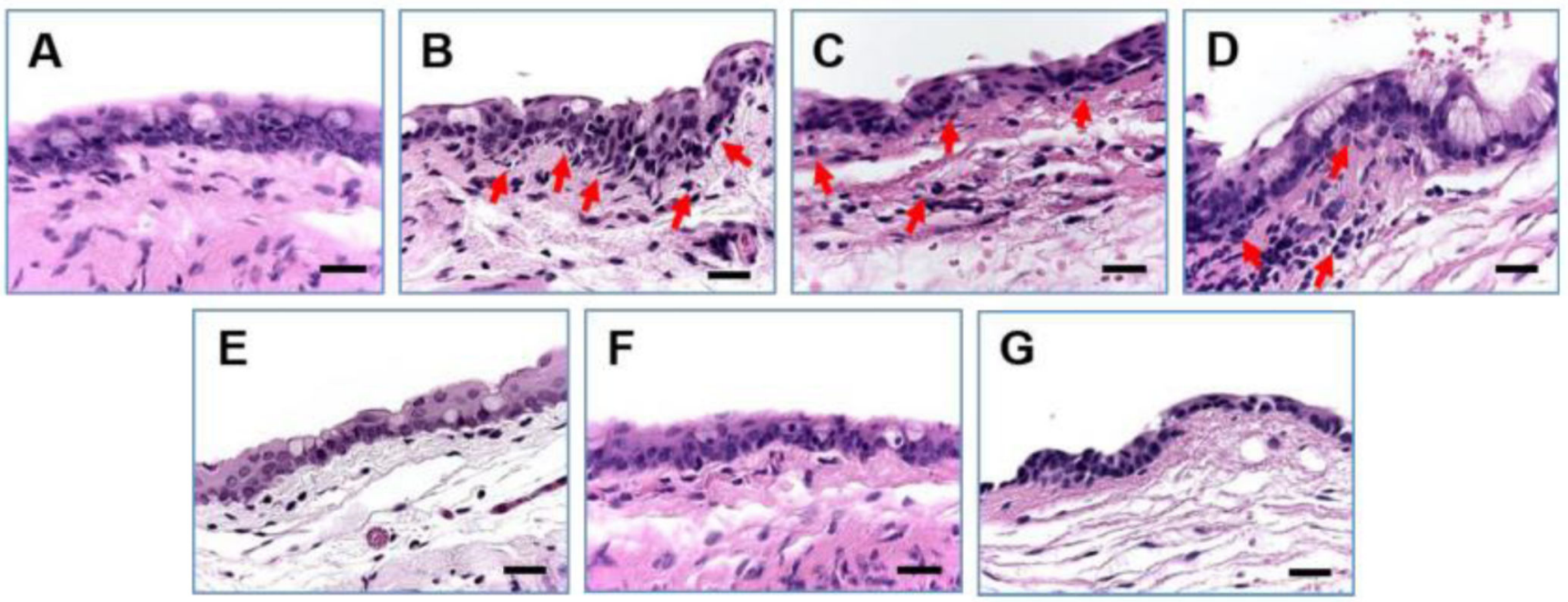

Fig. 12.

Histopathology analysis of ocular tissues of mice after seven different treatment types: (a) healthy, experimental dry eye treated with (b) Saline (x1/week), (c) Saline (x2/week), (d) Blank nanoparticles, (e) nanoparticle+ CsA (x1/week), (f) nanoparticle+CsA (x2/week), and (g) RESTASIS (x3/day). The scale bars (black) are $300 \mu$ min length. The arrows (red) represent some of the inflammatory infiltrates such as lymphocytes, polymorphonuclears and eosinophils observed. [Reprinted from "Pharmaceutical research, Phenylboronic acid modified mucoadhesive nanoparticle drug carriers facilitate weekly treatment of experimentally induced dry eye syndrome, 8(2), (2015) 621-635. Liu, S., Chang, C. N., Verma, M. S., Hileeto, D., Muntz, A., Stahl, U., Woods, J., Jones, W., Gu, F. X. () Tsinghua University Press and Springer-Verlag Berlin Heidelberg 2014" With permission of Springer.]

Adv Drug Deliv Rev. Author manuscript; available in PMC 2018 December 01. 
A
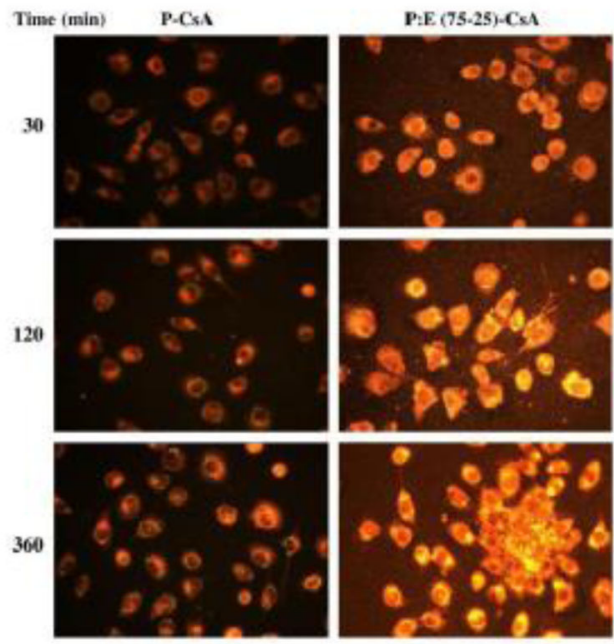

B

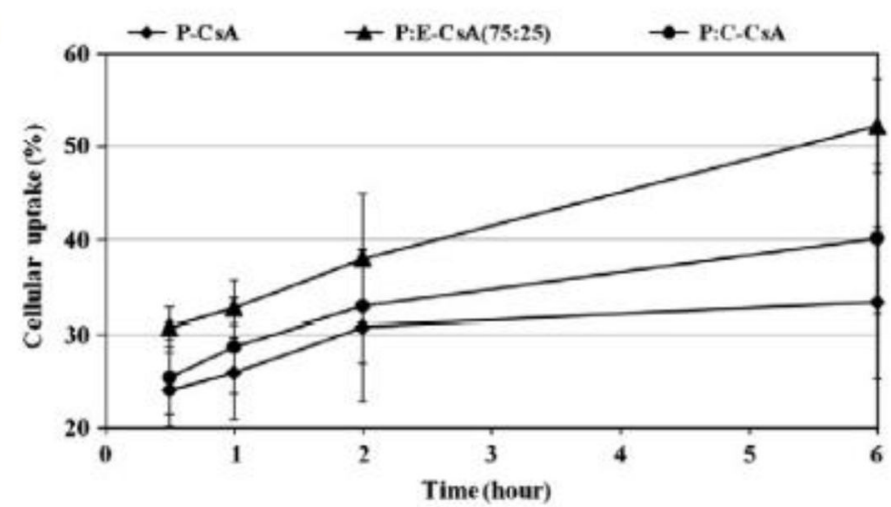

Fig. 13.

Time-dependent nanoparticles uptake by L929 (mouse fibroblast) cells. Photographs of representative series of cells exposed to $0.5 \mathrm{mg} / \mathrm{mL}$ NPs for 30, 120 and $360 \mathrm{~min}$. Original magnification 400× (A). Cellular uptake efficiency (\%) of Nile red labeled nanoparticles (B). [Reprinted from Journal of controlled release 151.3 (2011). Aksungur, P., Demirbilek, M., Denkbaş, E. B., Vandervoort, J., Ludwig, A., \& Unlu, N. Development and characterization of Cyclosporine A loaded nanoparticles for ocular drug delivery: Cellular toxicity, uptake, and kinetic studies. 286-294. Copyright () 2011 Elsevier B.V. with permission from Elsevier.] 


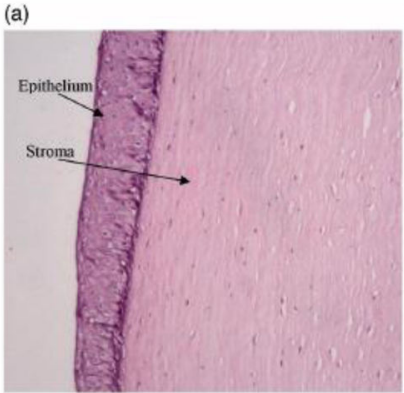

(b)

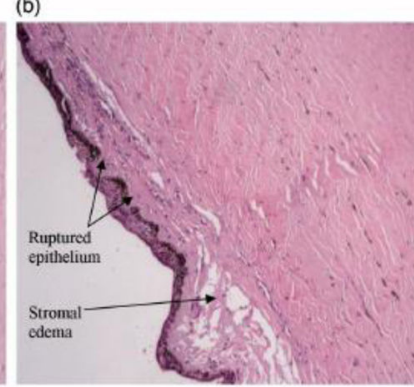

(c)

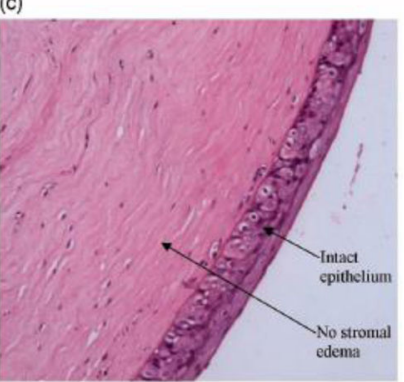

Fig. 14.

Images of histopathology of isolated cornea after treatment with (a) Control $(0.9 \% \mathrm{NaCl})$, (b) Irritant $(0.1 \mathrm{~N} \mathrm{NaOH})$ and (c) SLN. Irritant treated cornea showed a severely injured epithelial layer, which was separated from the Bowman's layer at various regions of the cornea, and showed a significant corneal swelling. The control and SLN-treated cornea displayed minimal or insignificant swelling. [Reprinted form Kumar, Rakesh, and Vivek Ranjan Sinha. "Solid lipid nanoparticle: an efficient carrier for improved ocular permeation of voriconazole." Drug development and industrial pharmacy 42.12 (2016): 1956-1967. () 2016 Informa UK Limited, trading as Taylor \& Francis Group. With permission from Taylor $\&$ Francis Ltd (www.tandfonline.com)] 


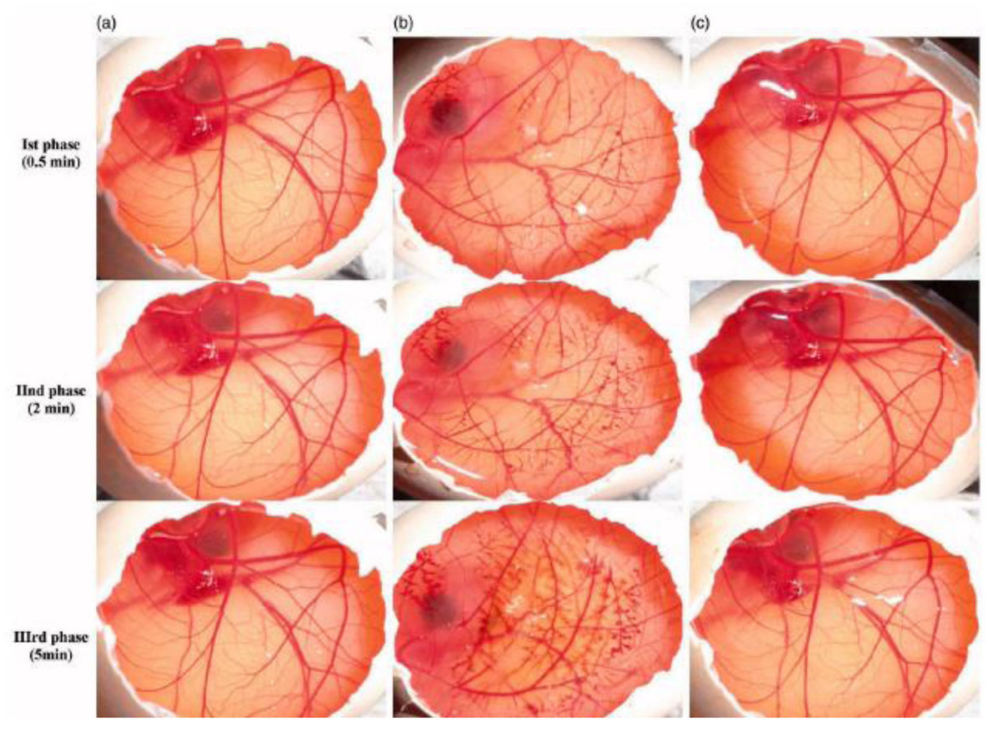

Fig. 15.

Images of HETCAM assay after treatment with (a) Control $(0.9 \% \mathrm{NaCl})$, (b) Irritant $(0.1 \mathrm{~N}$ $\mathrm{NaOH}$ ) and (c) SLN. Irritant treated showed a significant lysis, hemorrhage, and coagulation while the control and SLN-treated displayed non-irritant property. [Reprinted from Kumar, Rakesh, and Vivek Ranjan Sinha. "Solid lipid nanoparticle: an efficient carrier for improved ocular permeation of voriconazole." Drug development and industrial pharmacy 42.12 (2016): 1956-1967. (C) 2016 Informa UK Limited, trading as Taylor \& Francis Group. With permission from Taylor \& Francis Ltd (www.tandfonline.com)] 


\section{Table 1}

Administration routes for delivering drugs to the anterior segment of the eye.

\begin{tabular}{|c|c|c|}
\hline Route of administration & Advantages & Limitation \\
\hline Topical & $\begin{array}{l}\text { simple, convenient, self-administrable and noninvasive } \\
\text { administration; avoiding the blood-aqueous barrier; no first- } \\
\text { pass metabolism }\end{array}$ & $\begin{array}{l}\text { short contact time of drug on the ocular surface; } \\
\text { low efficiency and low bioavailability due to } \\
\text { corneal and conjunctival barriers, tear clearance, } \\
\text { and nasolacrimal drainage }\end{array}$ \\
\hline Intracameral & $\begin{array}{l}\text { avoiding the cornea, conjunctiva and blood-aqueous barrier; } \\
\text { no first-pass metabolism; high efficacy; high bioavailability }\end{array}$ & $\begin{array}{l}\text { usually need reconstitution; correct dosing and } \\
\text { preparation are critical. }\end{array}$ \\
\hline Subconjunctival & $\begin{array}{l}\text { easy and minimally invasive administration; avoiding the } \\
\text { cornea and blood-aqueous barrier; no first-pass metabolism; } \\
\text { good efficacy; good bioavailability; sustained release }\end{array}$ & conjunctival blood and lymphatic clearance \\
\hline Systemic & $\begin{array}{l}\text { convenient to deliver a large dose of the drug; noninvasive; } \\
\text { avoiding the cornea }\end{array}$ & $\begin{array}{l}\text { low bioavailability due to systemic absorption and } \\
\text { blood-aqueous barrier; first-pass metabolism }\end{array}$ \\
\hline
\end{tabular}




\section{Table 2}

Summary of the techniques used for physicochemical and in vitro and in vivo characterizations of nanoparticles for anterior ocular delivery.

\begin{tabular}{|c|c|}
\hline Property & Characterization Method \\
\hline Yield & Gravimetric [53] \\
\hline \multirow[t]{6}{*}{ Particle size/morphology } & $\begin{array}{l}\text { Dynamic light scattering }[25,53,91,95,118,119,123,142,150,171,174,178,185 \text {, } \\
193,194,210,213,246-255]\end{array}$ \\
\hline & $\begin{array}{l}\text { Transmission electron microscopy (TEM) }[30,91,102,113,118,119,174,178,191 \text {, } \\
193,194,208,248,256-260]\end{array}$ \\
\hline & Scanning electron microscopy (SEM) $[117,119,218,251,253,261,262]$ \\
\hline & Field Emission Scanning Electron Microscope [210] \\
\hline & Thermomicroscopy [263] \\
\hline & Atomic microscopy [228] \\
\hline Zeta potential & $\begin{array}{l}\text { Laser Doppler anemometry (LDA)/electrophoretic mobility }[25,95,119,123,142,174 \text {, } \\
185,248,249,252-255]\end{array}$ \\
\hline \multirow[t]{4}{*}{ Chemical characterization } & $\begin{array}{l}\text { Fourier Transform Infrared (FTIR) spectroscopy }[91,95,113,117,142,208,259,261, \\
263-265]\end{array}$ \\
\hline & Nuclear magnetic resonance (NMR) spectroscopy $[203,208,259,264]$ \\
\hline & Mass spectrometry [266] \\
\hline & Elemental analysis $[251,264]$ \\
\hline \multirow[t]{4}{*}{ Solid-state characterization } & Infrared spectrophotometry $[142,261,263]$ \\
\hline & $\mathrm{X}$-ray diffractometry $[91,113,142,171,263,267]$ \\
\hline & $\begin{array}{l}\text { Differential scanning calorimetry (DSC) }[91,95,113,142,171,174,191,193,218, \\
259,263,267]\end{array}$ \\
\hline & Thermal gravimetric analysis (TGA) [261] \\
\hline Molecular weight characterization & Size exclusion chromatography [203] \\
\hline Surface tension & Tensiometer [203] \\
\hline Viscosity of solution & Rheometer/viscometer $[185,203,260,265,268]$ \\
\hline \multirow[t]{5}{*}{ Drug loading and Entrapment efficiency } & HPLC $[25,89,171,174,193,194,208,221,249,250,254]$ \\
\hline & $\begin{array}{l}\text { UV-Vis spectroscopy }[91,102,113,117-119,178,210,253,255,258,260,262,264 \text {, } \\
\text { 267] }\end{array}$ \\
\hline & Liquid scintillation counter for radio labelled[123] \\
\hline & bioassay [252] \\
\hline & $\begin{array}{l}\text { GPC and UV [213] } \\
\text { (drug separated by dialysis or centrifugation or membrane filtration) }\end{array}$ \\
\hline \multirow[t]{4}{*}{ In vitro drug release } & Sample and Separate $[25,95,117,119,123,142,150,210,269]$ \\
\hline & $\begin{array}{l}\text { Dialysis system }[102,113,150,178,191,193-195,208,246-249,253,255,259-263 \text {, } \\
265,267]\end{array}$ \\
\hline & Diffusion cell $[171,213,268]$ \\
\hline & $\begin{array}{l}\text { (drug analysis by spectrofluorometric or UV-Vis or HPLC or LC-MS/MS or liquid } \\
\text { scintillation counting) }\end{array}$ \\
\hline Drug stability & HPLC $[208,210,218,265,270]$ \\
\hline \multirow[t]{2}{*}{ Enzyme stability } & $\begin{array}{l}\text { Incubation with Mucin ad Lysozyme and analyze for particle size, Zeta and drug } \\
\text { leakage }[117,256]\end{array}$ \\
\hline & Incubation in simulated tear fluid and analyze for particle size [252] \\
\hline Storage stability & $\begin{array}{l}\text { Incubation at appropriate conditions and analyze for particle size, poly-dispersity index, } \\
\text { zeta potential and/or drug leakage }[30,142,179,208,255,261,262,265,271]\end{array}$ \\
\hline
\end{tabular}

Adv Drug Deliv Rev. Author manuscript; available in PMC 2018 December 01. 


\begin{tabular}{|c|c|}
\hline Property & Characterization Method \\
\hline \multirow[t]{5}{*}{ In vitro cytotoxicity } & MTT colorimetric assay or XTT colorimetric assay $[102,230,252][30]$ \\
\hline & SEM [256] \\
\hline & Neutral red (NR) assay[174] \\
\hline & Erythrocyte morphology \& Hemolysis/spectrophotometer[208] \\
\hline & Resazurin assay[258] \\
\hline In vitro irritancy test & $\begin{array}{l}\text { Hen's Egg Test - Chorioallantoic Membrane (HET-CAM) test [113, 178, 218, 258, 259, } \\
262,267,268]\end{array}$ \\
\hline \multirow[t]{2}{*}{ Mucoadhesive capacity } & $\begin{array}{l}\text { Mucin-particle method (incubating with mucin-centrifugation-analyze free mucin by } \\
\text { colorimetric method) }[91,113,185,258,259,267]\end{array}$ \\
\hline & Ex vivo study by fluorescence microscopy [117] \\
\hline In vitro ocular permeability & $\begin{array}{l}\text { Cell Monolayers - Transwell Inserts [171, 174] } \\
\text { (drug analysis by HPLC or LC-MS/MS) }\end{array}$ \\
\hline Ex vivo ocular permeability & $\begin{array}{l}\text { Excised cornea and a diffusion apparatus [30, 178, 191, 193-195, 213, 254] } \\
\text { (drug analysis by HPLC or LC-MS/MS) }\end{array}$ \\
\hline Ex vivo tissue hydration & Gravimetric -Wet and dry weight (desiccation) $[178,213]$ \\
\hline \multirow[t]{4}{*}{$\begin{array}{l}\text { Cellular internalization or uptake (in vitro or } e x \\
\text { vivo) }\end{array}$} & $\begin{array}{l}\text { Immunofluorescence Assays by confocal laser scanning microscope }[113,174,178 \text {, } \\
218,247,252,256,272]\end{array}$ \\
\hline & Spectrofluorimetry $[256,272]$ \\
\hline & Fluorescence microscopy $[230,272]$ \\
\hline & Flow cytometry [128] \\
\hline \multirow[t]{3}{*}{ In vivo ocular tolerability } & Modified Draize Test using a slit-lamp[25, 142, 193, 273] \\
\hline & Histopathology $[30,150,194]$ \\
\hline & $\begin{array}{l}\text { Slit lamp examination of clinical observations and ocular reactions (such as swelling } \\
\text { and redness, conjunctival chemosis, discharge, iris and corneal lesions)-ocular irritation } \\
\text { scoring testing system }[150,185,203,208,213,252,254,255,272]\end{array}$ \\
\hline \multirow[t]{5}{*}{ In vivo precorneal retention } & HPLC [254] \\
\hline & LC-MS/MS analysis $[268,274]$ \\
\hline & $\begin{array}{l}\text { Slit-lamp examination of fluorescence or Fluorescence imaging system using dye [191, } \\
193,195,203,208,213,250]\end{array}$ \\
\hline & SEM [261] \\
\hline & Gamma scintigraphy $[113,179,267]$ \\
\hline \multirow[t]{6}{*}{ In vivo ocular drug distribution/ocular bioavailability } & Polarization fluoroimmunoanalysis [246] \\
\hline & Radio labelled - liquid scintillation counter $[118,123,248]$ \\
\hline & HPLC $[25,178,191,193,194,249,250,263,273]$ \\
\hline & UV-Vis [261] \\
\hline & single photon emission computed tomography image analysis [118] \\
\hline & microdialysis [195] \\
\hline
\end{tabular}

Adv Drug Deliv Rev. Author manuscript; available in PMC 2018 December 01. 


\section{Table 3}

Chemical structures of synthetic polymers used for drug delivery to the anterior segment of the eye.

\begin{tabular}{lll}
\hline Name & Chemical Structures \\
\hline Poly(alkyl-cyanoacrylate) &
\end{tabular}

Poly(lactide) (PLA)<smiles>CC(C)OC(C)C(=O)C(C)(C)C</smiles>

Poly(lactic-co-glycolic acid) (PLGA)

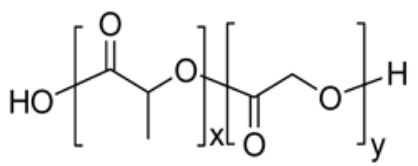

Poly( $\varepsilon$-caprolactone) (PCL)

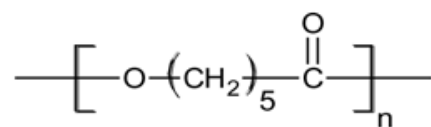

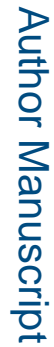

Poly(ethylene glycol) (PEG)

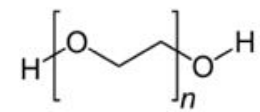

Poly(L-lysine $)$

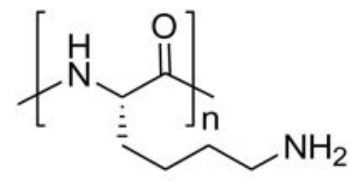

Carbopol $^{\circledR}$

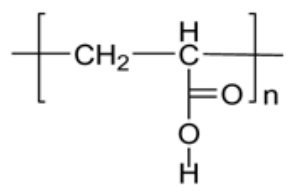




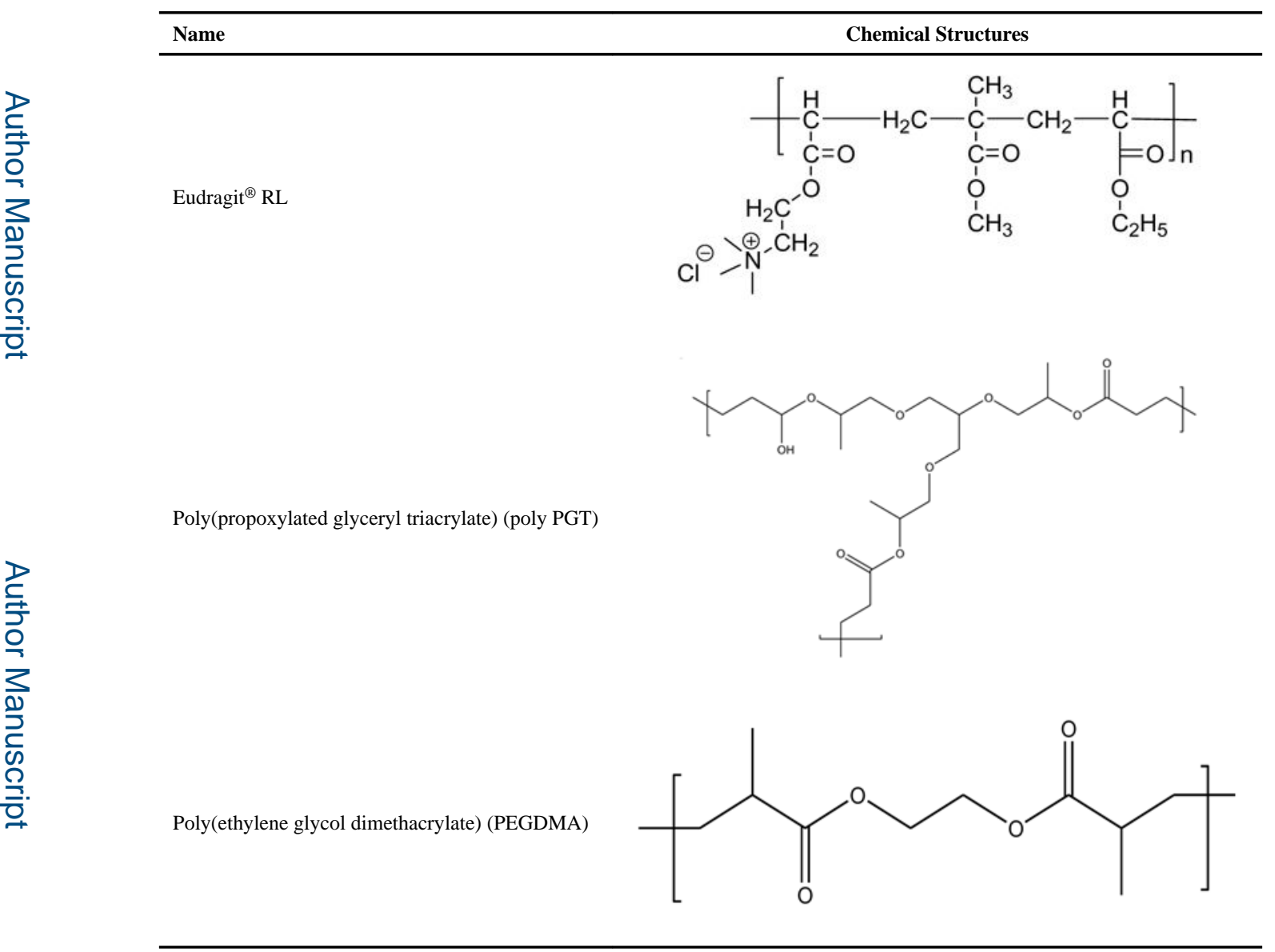

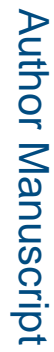

롬

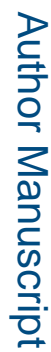


Table 4

Chemical structures of polysaccharides used for drug delivery to the anterior segment of the eye.

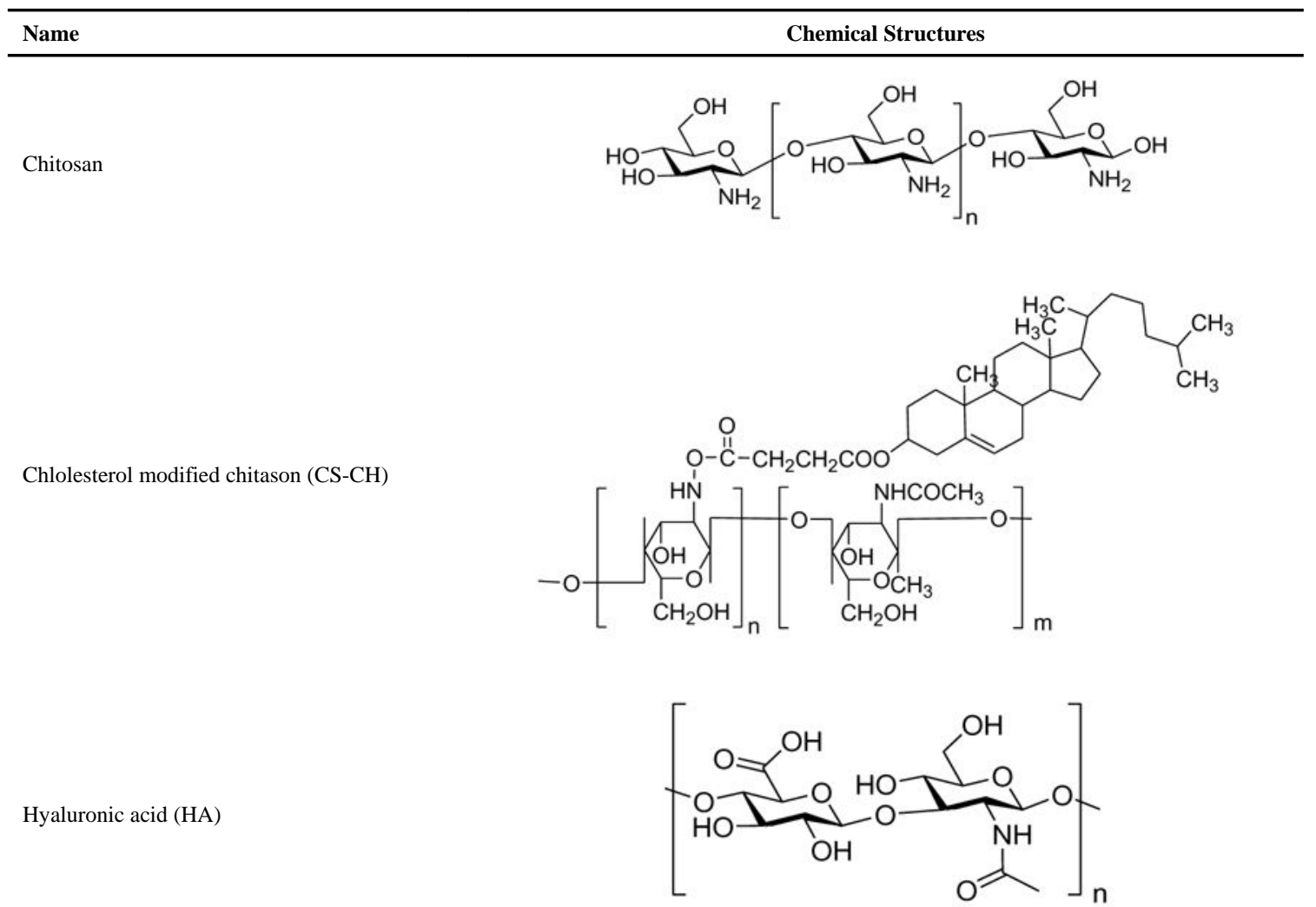

Sodium alginate

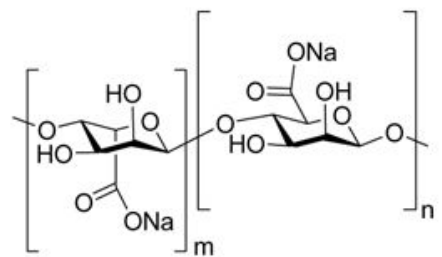

Sulfobutylether-cyclodextrin

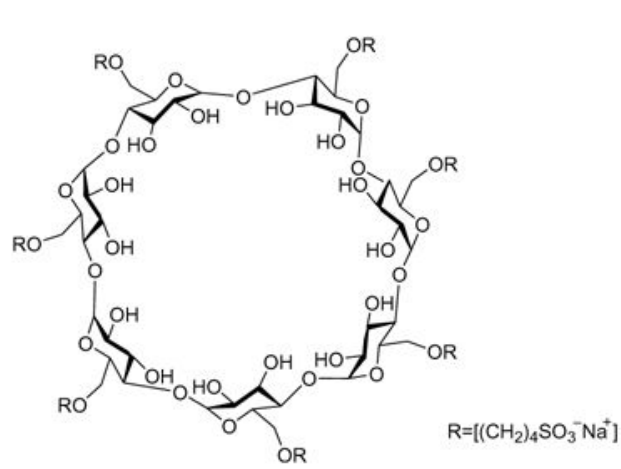

Adv Drug Deliv Rev. Author manuscript; available in PMC 2018 December 01. 

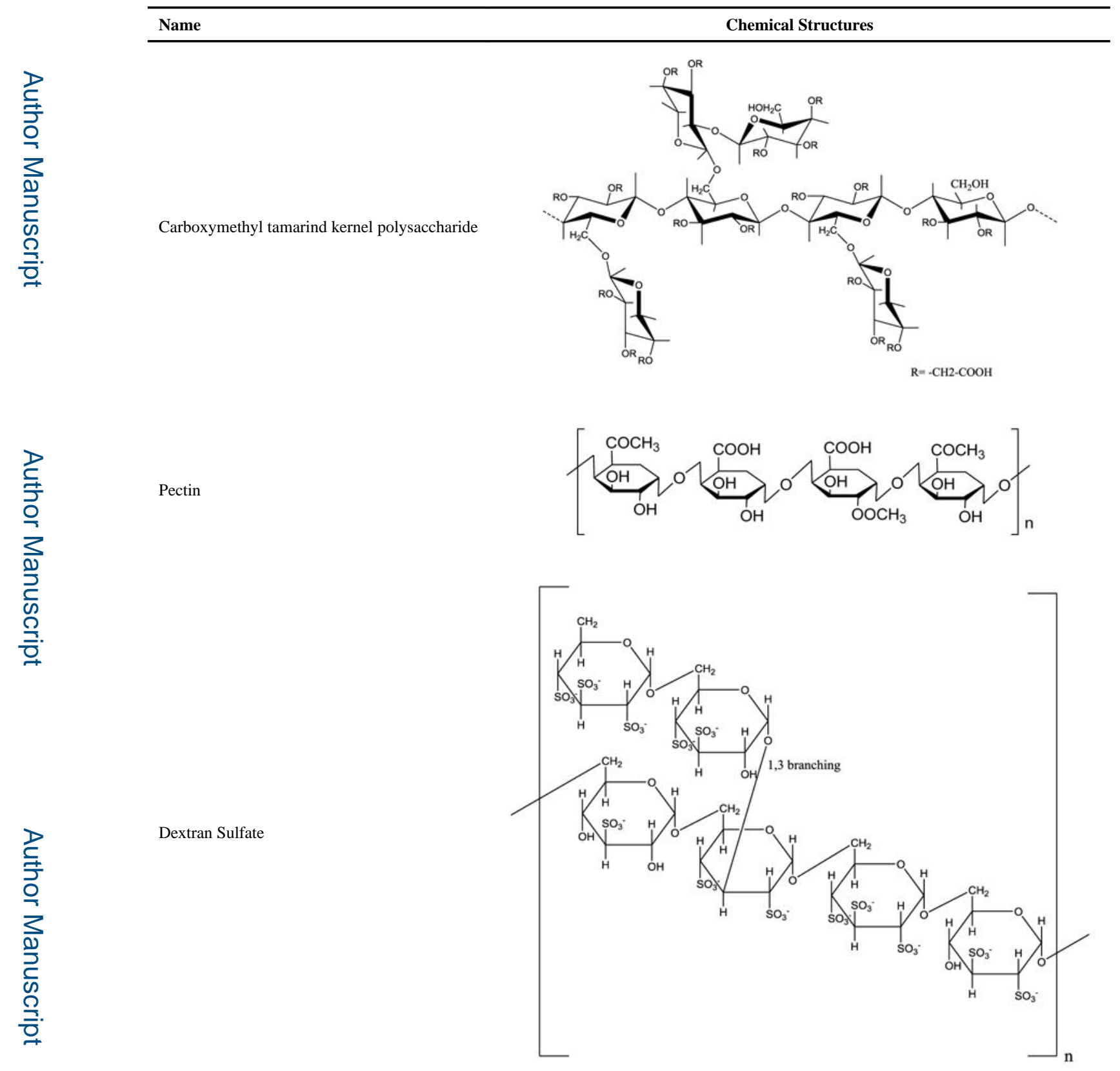

Gum Cordia

Natural anionic gum (Structure N/A)

Pullan

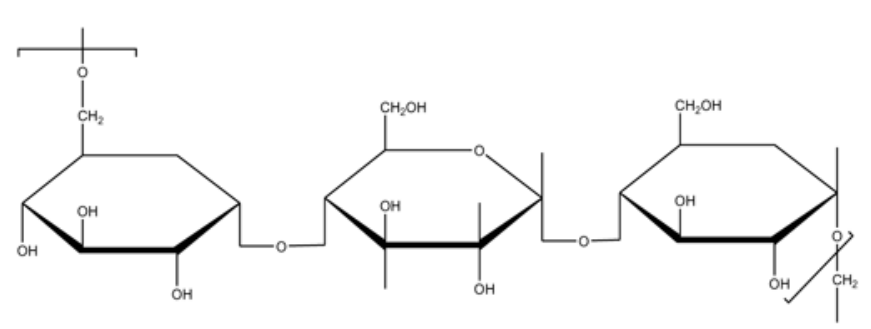

Adv Drug Deliv Rev. Author manuscript; available in PMC 2018 December 01. 


\section{Table 5}

Chemical structures of lipids used for drug delivery to the anterior segment of the eye.

\begin{tabular}{l} 
Name \\
\hline \\
Thiolated PEG stearate (cysteine-polyethylene glycol \\
monostearate)
\end{tabular}
monostearate)

Compritol 888 ATO (Behenoyl polyoxyl-8 glycerides NF) or Docosanoic acid,1,2,3-propanetriyl ester

Precirol ATO 5 (Glycerol Palmito-Stearate)

Miglyol 812

Dimyristoyl phosphatidylcholine

Gelucire 44/12 (Lauroyl polyoxyl-32 glycerides NF)

\section{Chemical Structures}
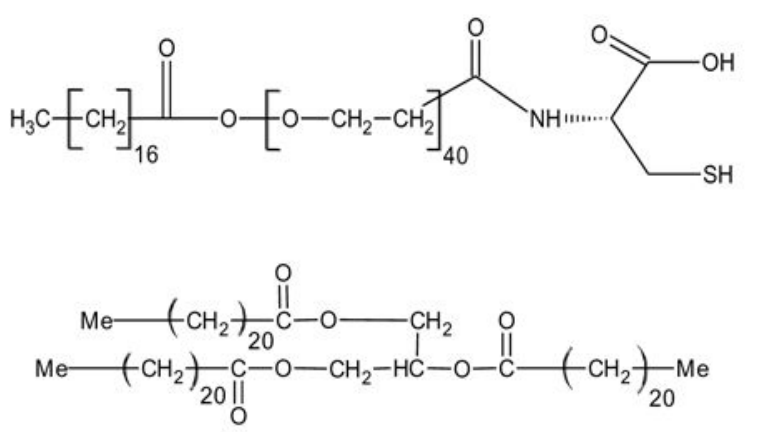

$$
\overbrace{\mathrm{OH}}^{\mathrm{OH}} \text { Glycerol }
$$
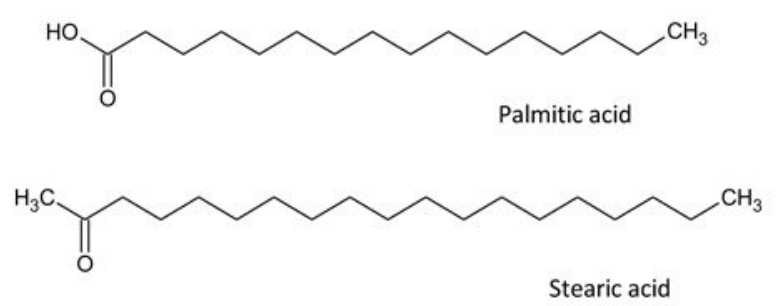

N/A

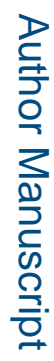
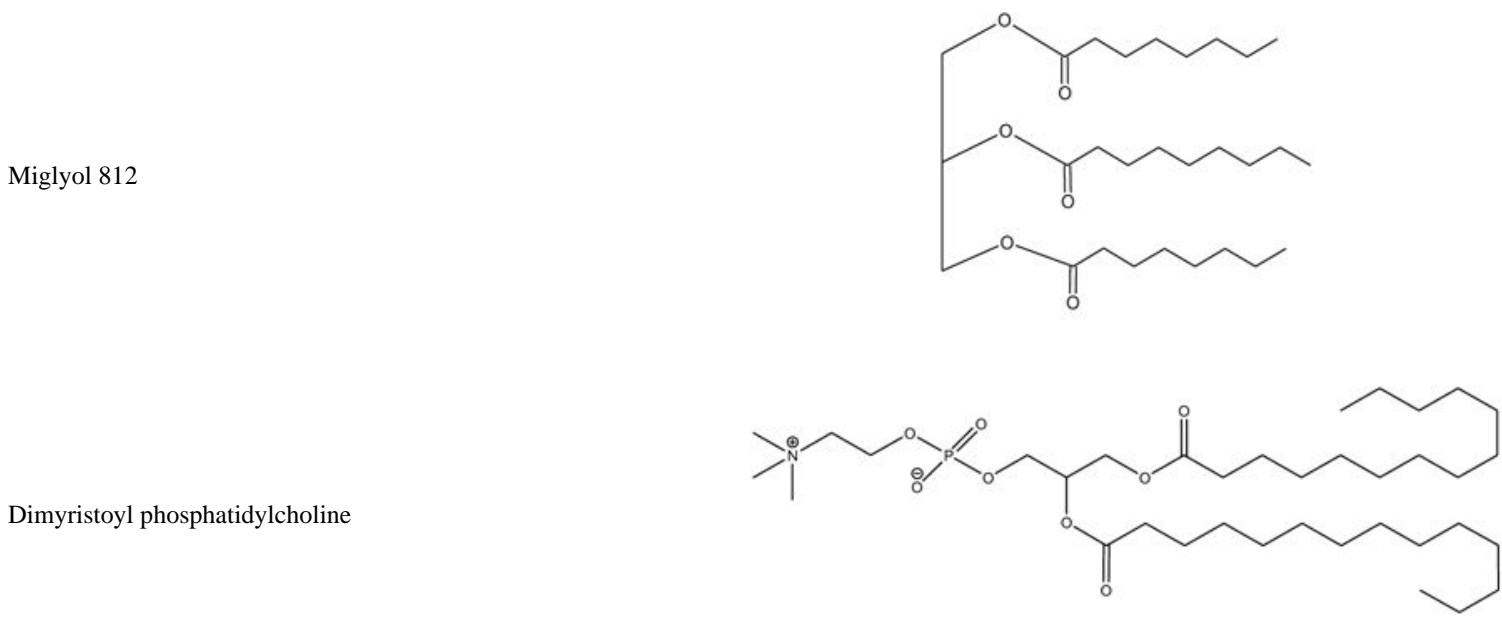


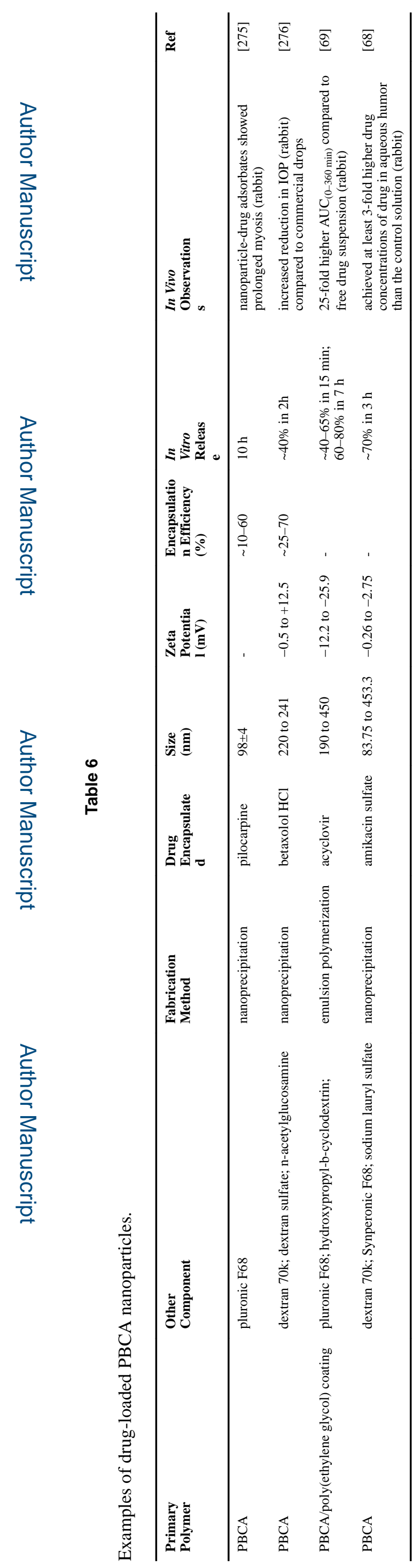




$$
\text { Whin }
$$




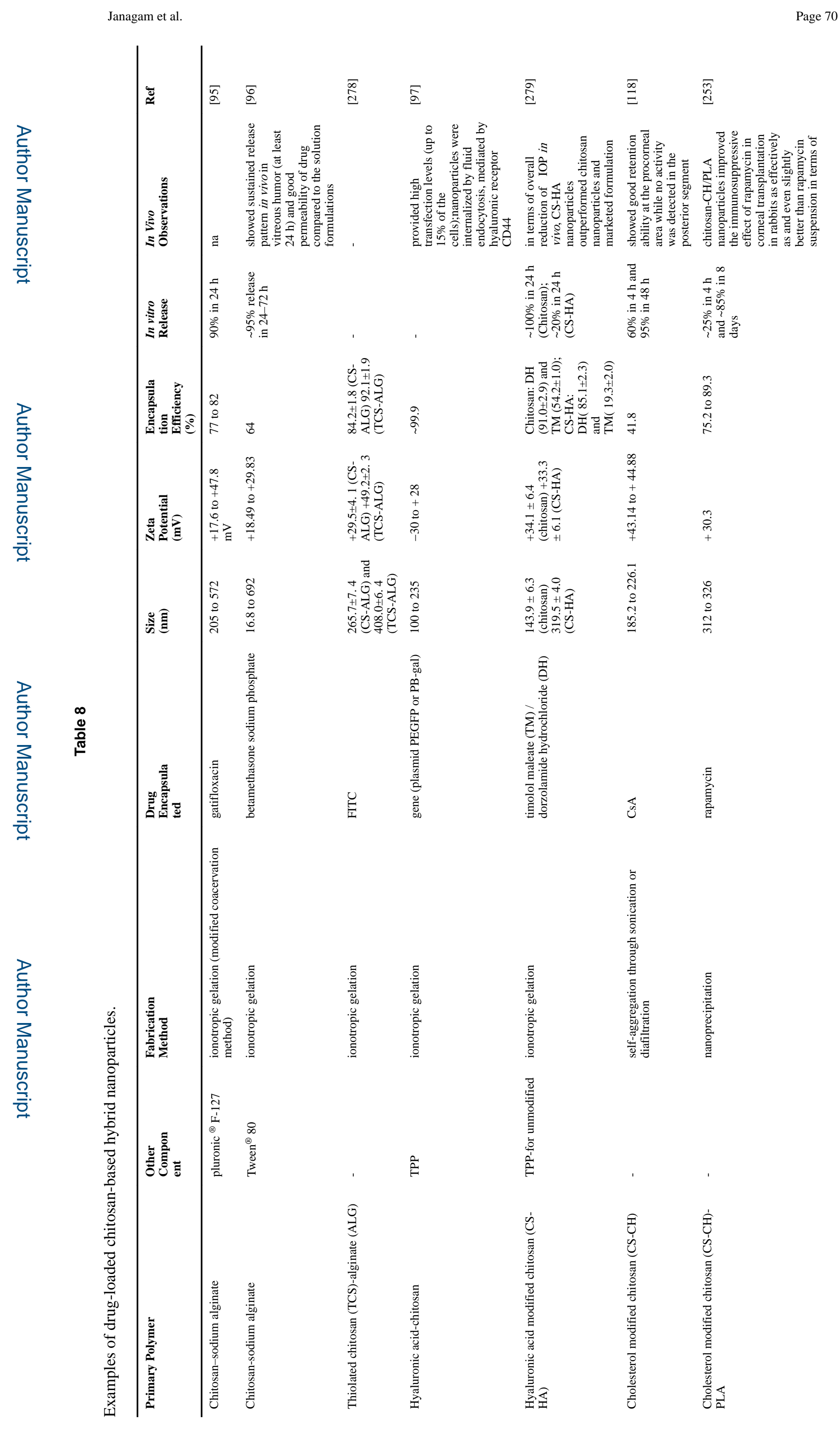




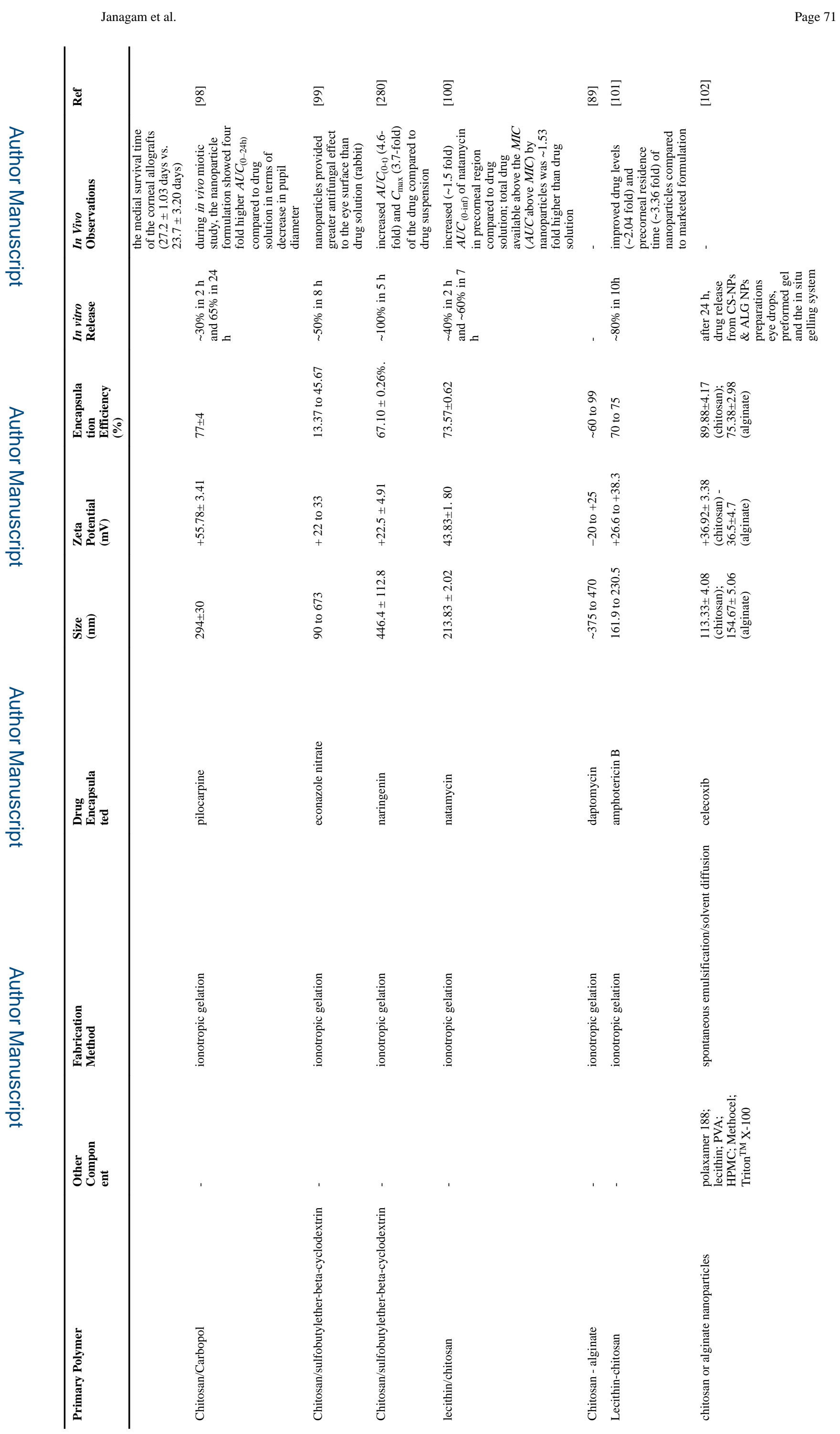

Adv Drug Deliv Rev. Author manuscript; available in PMC 2018 December 01. 


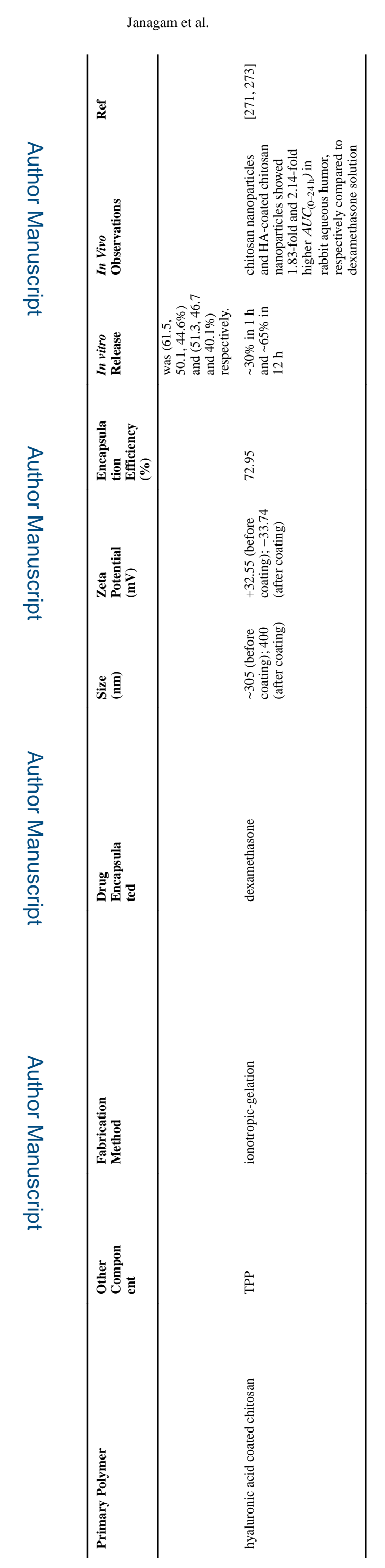

Page 72 


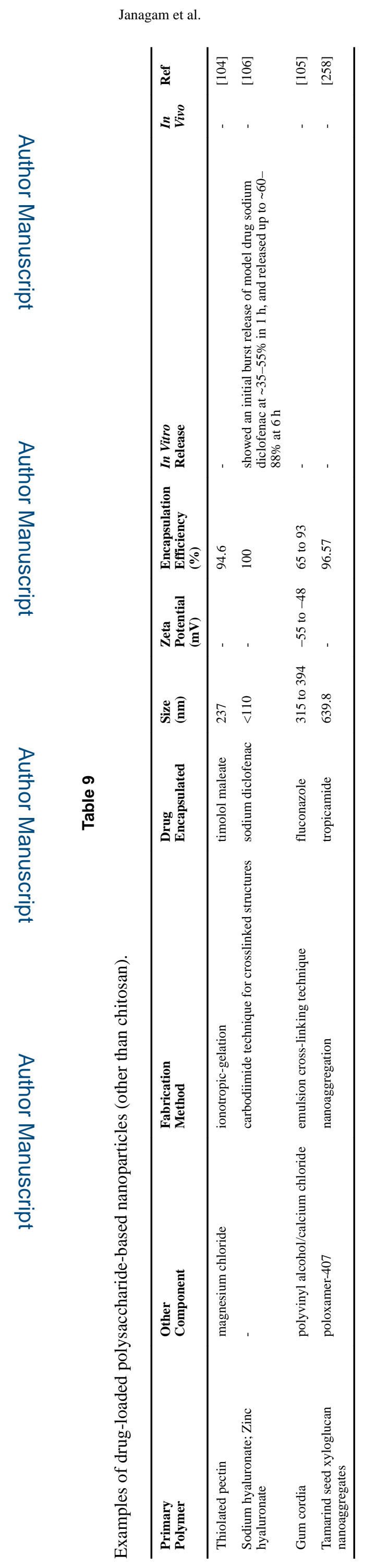




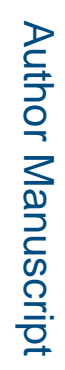

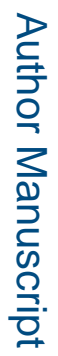

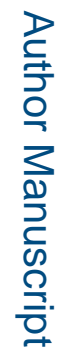

$\frac{2}{2+\frac{1}{2}}$

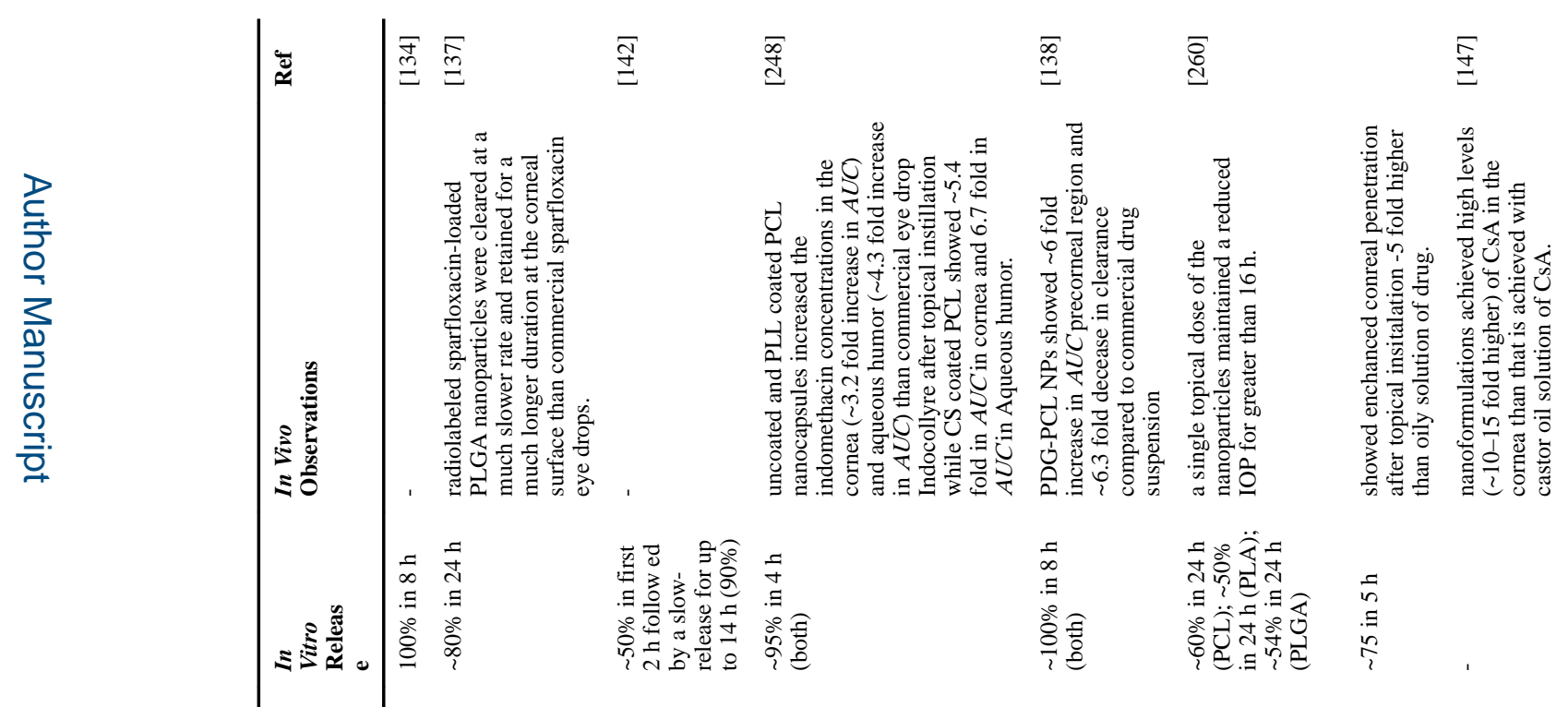

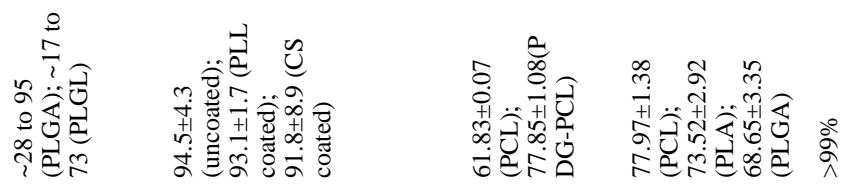

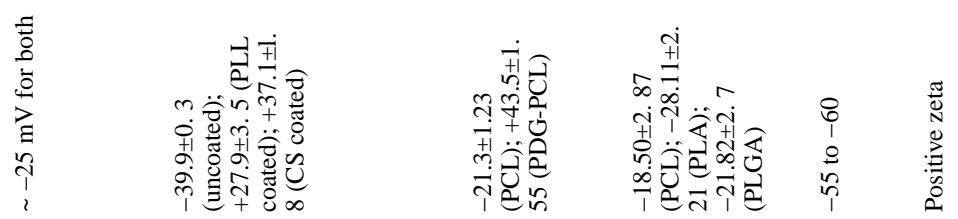

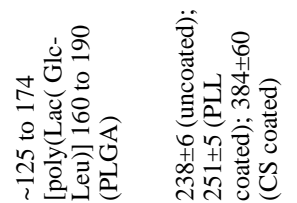

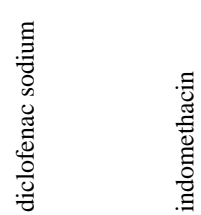

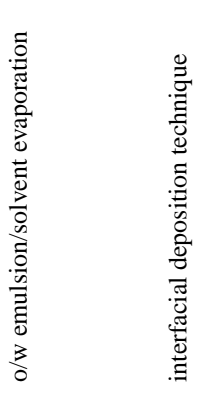

웅

$\frac{\otimes}{2}$

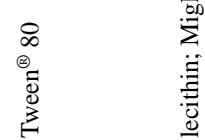

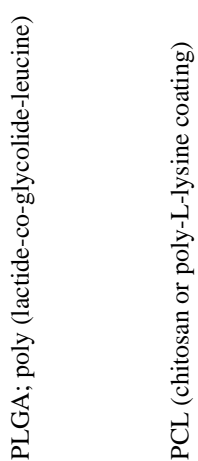

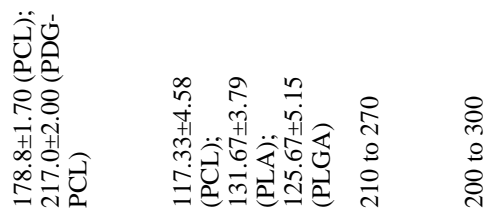

高高高

$\mathbb{8} \quad \overleftarrow{8}$

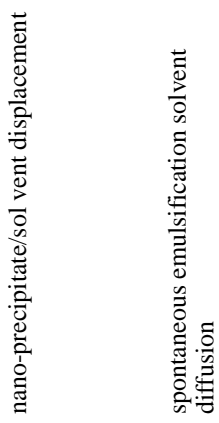

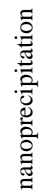

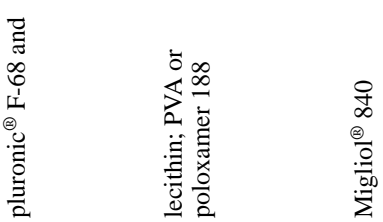

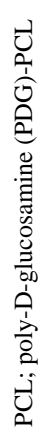

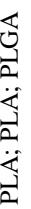

$\underset{殳}{己}$

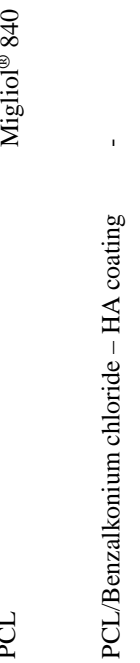





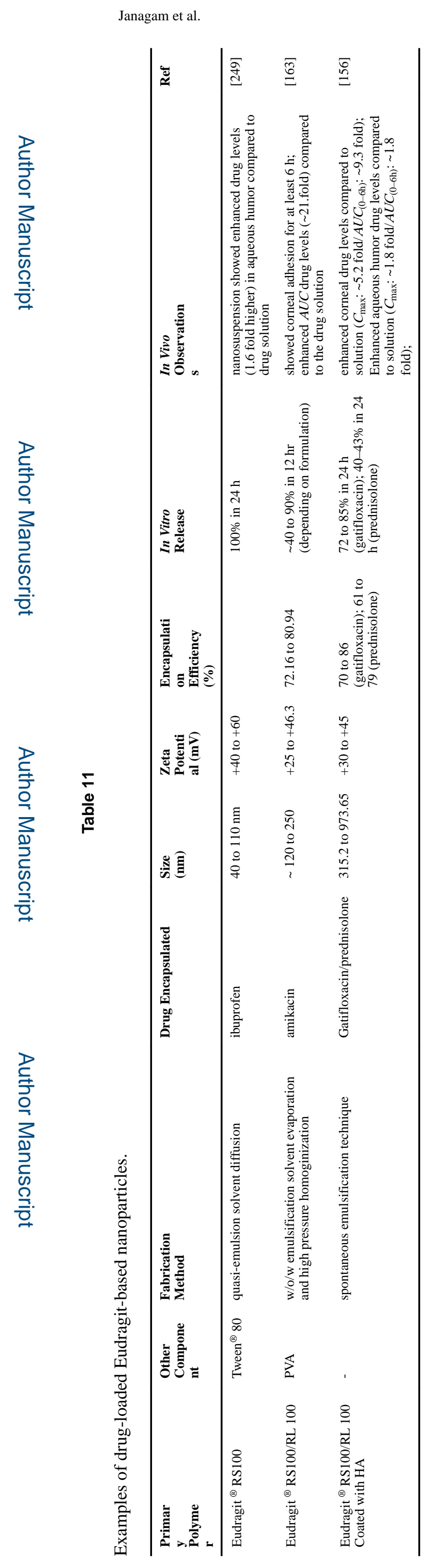




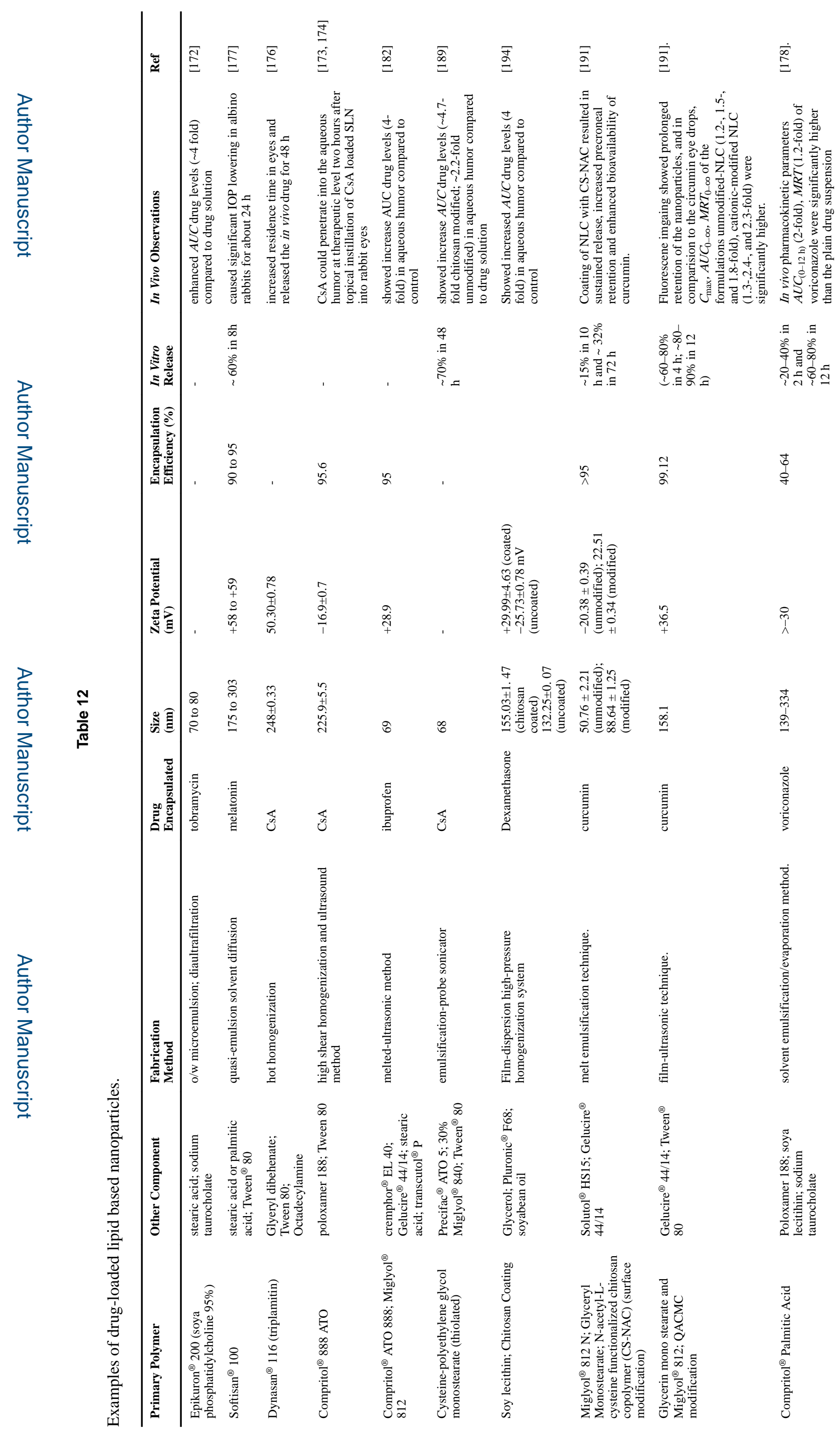




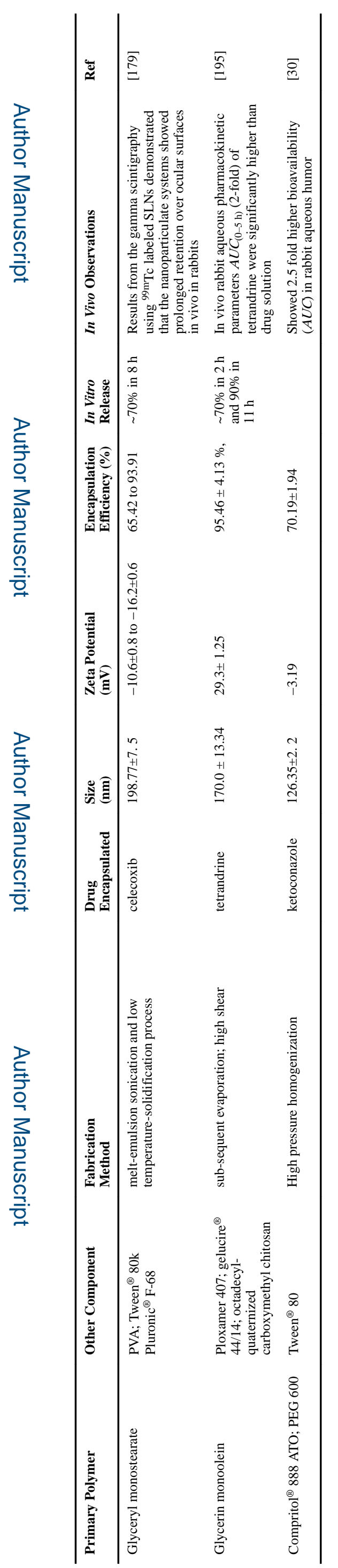




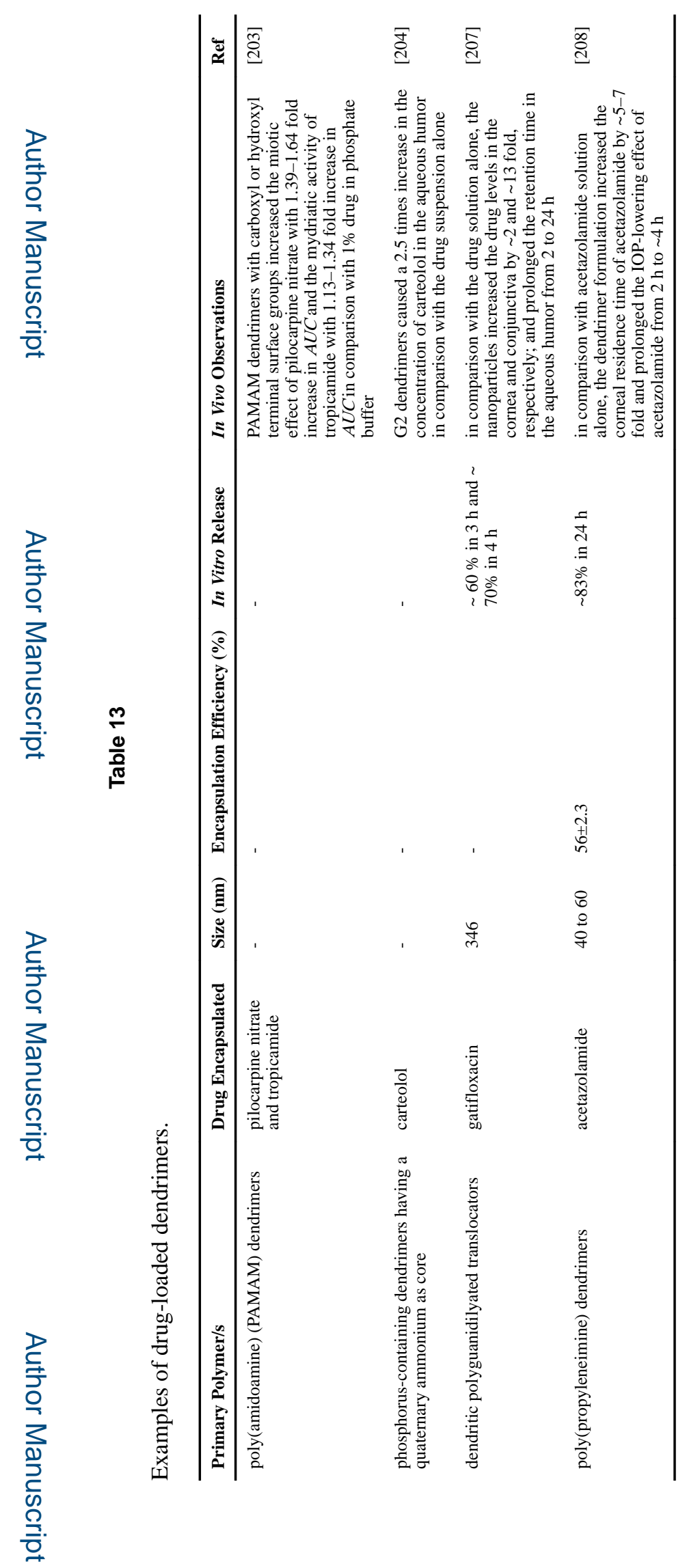

Adv Drug Deliv Rev. Author manuscript; available in PMC 2018 December 01. 


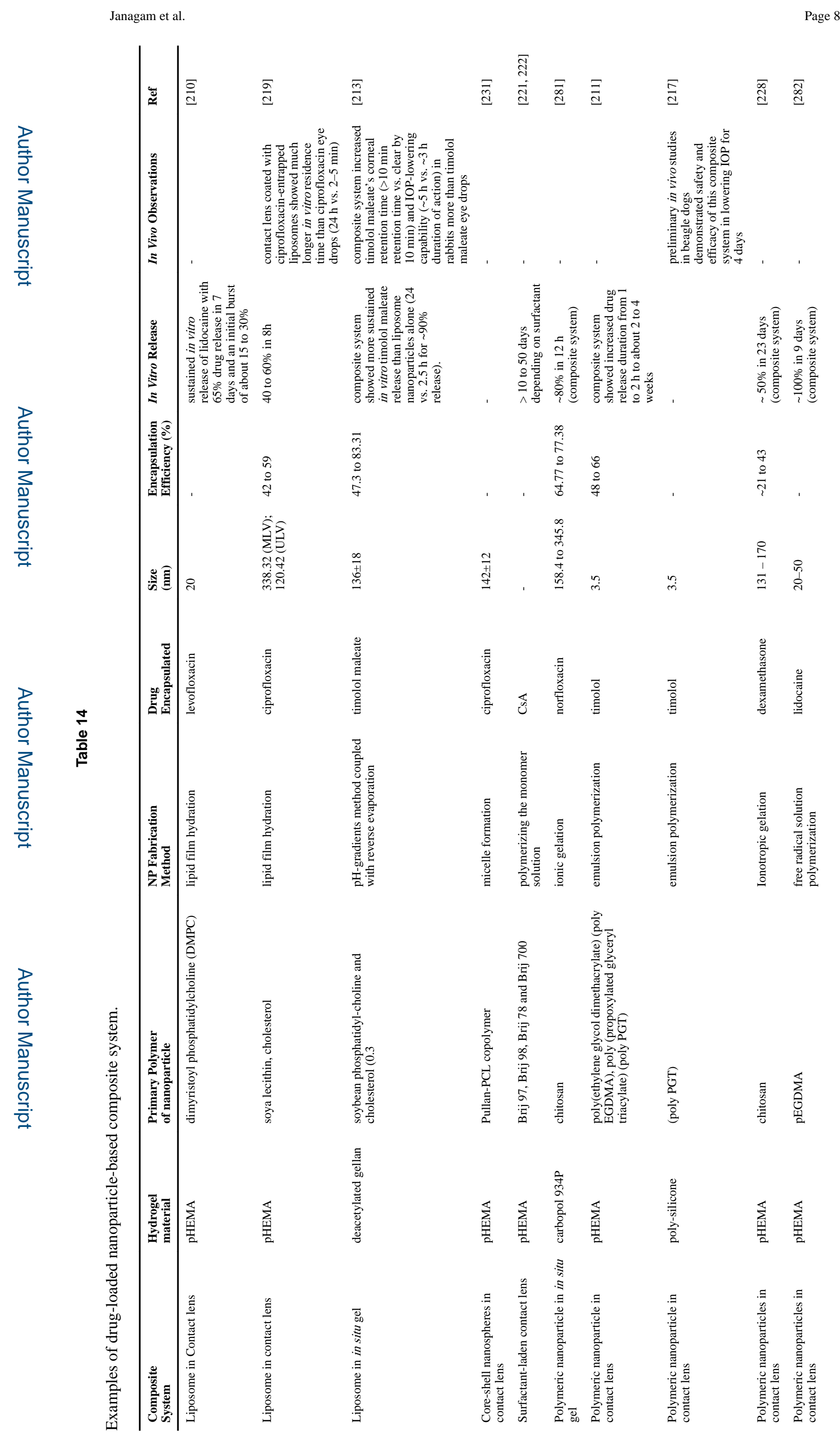




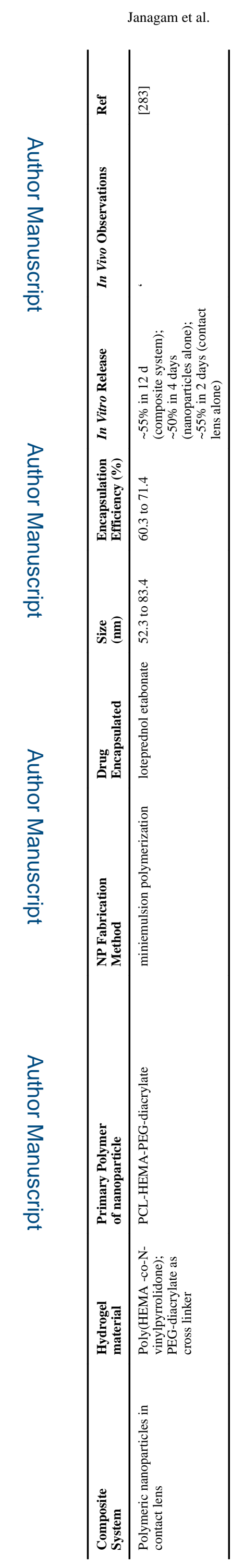

Adv Drug Deliv Rev. Author manuscript; available in PMC 2018 December 01. 